TRANSACTIONS OF THE

AMERICAN MATHEMATICAL SOCIETY

Volume 351, Number 4, April 1999, Pages 1293-1353

S 0002-9947(99)02120-0

\title{
THESE ARE THE DIFFERENTIALS OF ORDER $n$
}

\author{
DAN LAKSOV AND ANDERS THORUP
}

\begin{abstract}
We answer P.-A. Meyer's question "Qu'est ce qu'une différentielle d'ordre $n$ ?". In fact, we present a general theory of higher order differentials based upon a construction of universal objects for higher order differentials. Applied to successive tangent spaces on a differentiable manifold, our theory gives the higher order differentials of Meyer as well as several new results on differentials on differentiable manifolds. In addition our approach gives a natural explanation of the quite mysterious multiplicative structure on higher order differentials observed by Meyer. Applied to iterations of the first order Kähler differentials our theory gives an algebra of higher order differentials for any smooth scheme. We also observe that much of the recent work on higher order osculation spaces of varieties fits well into the framework of our theory.
\end{abstract}

\section{INTRODUCTION}

In an interesting, as well as entertaining, article [22] P.-A. Meyer asks the question "Qu'est ce qu'une différentielle d'ordre $n$ ?". He answers the question in the case of differentiable manifolds and comments on the history and uses of higher order differentials. One of the most intriguing observations that Meyer makes, see also $[21]$, is that the higher order differentials have a, quite mysterious, multiplicative structure.

The purpose of this article is to answer Meyer's question and to expand the theory so that it covers a wide area of applications from manifolds to schemes over an arbitrary base. We present a general theory of higher order differentials and, in particular, we give a natural explanation of their multiplicative structure. Our theory, when applied to successive tangent spaces on a differentiable manifold, gives the higher order differentials of Meyer, with their multiplicative structure. When applied to iterations of the first order Kähler differentials, it gives an algebra of higher order differentials for any smooth scheme.

The higher order differentials were part of the folklore of mathematics up to the end of the previous century, and formulas like

$$
d^{2} f=f_{x}^{\prime} d^{2} x+f_{y}^{\prime} d^{2} y+f_{x^{2}}^{\prime \prime} d x^{2}+2 f_{x y}^{\prime \prime} d x d y+f_{y^{2}}^{\prime \prime} d y^{2}
$$

can be found in most classical calculus books. According to Meyer, the higher order differentials vanished from the textbooks in our century because of the obscurity

Received by the editors January 30, 1997.

1991 Mathematics Subject Classification. Primary 13N05, 14F10; Secondary 16Sxx.

The first author was partially supported by The Göran Gustafsson Foundation for Research in Natural Sciences and Medicine.

The second author was supported in part by the Danish Natural Science Research Council, grant 11-7428.

(C)1999 American Mathematical Society 
of their definition, and because the extensive use of exterior differentials led mathematicians to believe that $d^{2}$ should always be zero. The higher order differentials have, however, a natural role in differential calculus and, as we shall see, in geometry, because they have many desirable properties that are not shared with the exterior differentials.

Meyer revived the theory because of its use in stochastic differential geometry. There are, however, many other reasons for providing a solid foundation for, and to develop, the theory of higher order differentials. Indeed, from the form of these differentials, involving all the partial derivatives up to a given order, and since they are invariant under coordinate change, they provide an excellent tool for treating topics that involve osculating spaces of manifolds. This explains why we several times have crossed the path of higher order differentials in our work [18], [19], [20] on Weierstrass points on higher dimensional spaces.

The construction of Semple bundles, used by Collino [7], Colley-Kennedy [5], [6], and Arrondo-Sols-Speiser [24] to study higher order contacts of manifolds, is completely analogous to constructions of higher order differentials in special cases (see Examples (5.5), (5.8), (5.9), (5.10)). J.-P. Demailly [8] uses a generalization of the Semple construction in the hyperplane case to construct projectivized jet bundles for directed manifolds. These bundles are essential in his study of hyperbolic projective varieties.

The symmetrization of our higher order differentials has been studied in various contexts. A construction of systems leading to higher order symmetric differentials is essential in the work of Iitaka [10], [11], [12], [13] on Weierstrass points (see Example (5.7)). In differential algebra a similar construction is used by Johnson [14], [15] and leads to what he calls the canonical prolongation sequence, which is the same as the ring constructed by Iitaka, or our ring of symmetric differentials, in the more general case when a non-trivial derivation of the base ring is allowed. Johnson gives a description of prolongation sequences in terms of universal properties, in the spirit of the present article. The global properties of the canonical prolongation sequence have been studied by Buium [1], [2], [3], [4]. A particularly interesting special case of the ring described above is the ring of differential polynomial functions considered and studied by Ritt [23] and Kolchin [16], [17].

Meyer considers the following setup for the equation (1) and its generalization to $m$ dimensions: Let $X$ be a differentiable manifold, say $X$ is an open subset of $\mathbf{R}^{m}$, with coordinate functions $x_{j}$. Let $A=\mathcal{C}^{\infty}(X)$ be the ring of $k$-valued $\mathcal{C}^{\infty}$-functions on $X$, where $k$ is $\mathbf{R}$ or $\mathbf{C}$. Then, for any function $f$ in $A$, we have the formula for the differential,

$$
d f=\sum_{j=1}^{m} f_{x_{j}}^{\prime} d x_{j},
$$

which defines the partial derivatives $f_{x_{j}}^{\prime}$. The differential $d f$ may be viewed as a function on the tangent space $T X$. Consider, inductively, the iterated tangent space $T^{n+1} X=T T^{n} X$. Let $C_{n}:=\mathcal{C}^{\infty}\left(T^{n} X\right)$ be the algebra of $\mathcal{C}^{\infty}$-functions on $T^{n} X$. Then the differential of $\mathcal{C}^{\infty}$-functions on $T^{n} X$ may be viewed as a $k$-linear map $d: C_{n} \rightarrow C_{n+1}$, and we obtain a sequence of $k$-linear maps,

$$
A=C_{0} \stackrel{d}{\rightarrow} C_{1} \stackrel{d}{\rightarrow} C_{2} \stackrel{d}{\rightarrow} \cdots .
$$


In addition, there is a map of algebras $\iota: C_{n} \rightarrow C_{n+1}$, obtained by composing functions on $T^{n} X$ with the projection $T^{n+1} X \rightarrow T^{n} X$. In particular, each algebra $C_{n}$ is an $A$-module. Let $\Omega^{n}$ be the $A$-submodule of $C_{n}$ defined as follows: $\Omega^{0}:=A$ and, inductively, $\Omega^{n+1}$ is the $A$-submodule of $C_{n+1}$ generated by all differentials $d \omega$ for $\omega \in \Omega^{n}$. Then, from the maps (3), we obtain by restriction a sequence of $k$-linear maps,

$$
A=\Omega^{0} \stackrel{d}{\rightarrow} \Omega^{1} \stackrel{d}{\rightarrow} \Omega^{2} \stackrel{d}{\rightarrow} \cdots .
$$

Let $C$ be the direct sum of the $A$-modules $C_{n}$, and let $\Omega$ be the direct sum of the submodules $\Omega^{n}$.

The fundamental assertions of Meyer are the following: There is a natural product on the graded $A$-module $\Omega$,

$$
\Omega^{p} \otimes_{k} \Omega^{n} \rightarrow \Omega^{p+n}, \quad \text { denoted } \omega \otimes \pi \mapsto \omega \cdot \pi,
$$

such that, for $p=0$, the product is the natural product coming from the structure of $\Omega^{n}$ as an $A$-module and such that the maps $d$ of (4) form a derivation of $\Omega$ with respect to the product, that is, such that we have the equation

$$
d(\omega \cdot \pi)=d \omega \cdot \pi+\omega \cdot d \pi .
$$

Moreover, the product is associative. In fact, with the product (5), the $A$-module $\Omega$ is a ring containing $A=\Omega^{0}$ as a subring.

The equation (6) determines the product inductively with respect to the degree $p$ of $\omega$. For instance, for $p=1$, we use the equation $d(f \pi)=d f . \pi+f d \pi$ to obtain the equation

$$
d f \cdot \pi=d(f \pi)-f d \pi .
$$

The equation (7) determines the product for $p=1$, since any form $\omega$ in $\Omega^{1}$ is an $A$-linear combination of differentials $d f$.

If the derivation $d$ is applied to the equation (2), we obtain in $\Omega^{2}$ the equation

$$
\begin{aligned}
d^{2} f & =\sum_{j=1}^{m} d\left(f_{x_{j}}^{\prime} d x_{j}\right)=\sum_{j=1}^{m}\left(d f_{x_{j}}^{\prime} \cdot d x_{j}+f_{x_{j}}^{\prime} d^{2} x_{j}\right) \\
& =\sum_{i, j=1}^{m} f_{x_{j} x_{i}}^{\prime \prime} d x_{i} \cdot d x_{j}+\sum_{j=1}^{m} f_{x_{j}}^{\prime} d^{2} x_{j} .
\end{aligned}
$$

Note in particular that the product $d x_{i} . d x_{j}$ appearing in (8) is given by the formula in (7).

It should be emphasized that the product in $\Omega$ is not commutative. Hence the formula (8) is not the $m$-dimensional generalization of the formula (1). To obtain such a generalization, we have to divide the algebra $\Omega$ by its commutator ideal.

The analogous system in the algebraic case is constructed as follows: Take $C_{0}:=$ $A$ and, inductively, let $C_{n+1}$ be the symmetric algebra $C_{n+1}:=\operatorname{Sym}_{C_{n}}\left(\Omega_{C_{n} / k}^{1}\right)$ and let $d: C_{n} \rightarrow C_{n+1}$ be defined as the universal derivation $d: C_{n} \rightarrow \Omega_{C_{n} / k}^{1}$ followed by the inclusion of $\Omega_{C_{n} / k}^{1}$ into $C_{n+1}$. View $C_{n}$ as an $A$-module via the composition of the inclusions of algebras $C_{i} \rightarrow C_{i+1}$ for $i=0, \ldots, n-1$. The latter construction is a non-commutative version of the construction given by litaka that we mentioned above. Like Meyer we find that the theory becomes simpler and more transparent when we impose as few commutativity conditions as possible. 
The results in this article grew out of an attempt to give a natural explanation of the assertions made by Meyer, in a general setting that includes the algebraic analogue. We consider, for any $k$-algebra $A$, general systems $(C, d)$ of the form $(3)$, consisting of $A$-modules $C_{n}$ and a sequence of $k$-linear maps,

$$
A=C_{0} \stackrel{d}{\rightarrow} C_{1} \stackrel{d}{\rightarrow} C_{2} \stackrel{d}{\rightarrow} \cdots .
$$

With such a system we associate, as in (4), a corresponding sequence of $k$-linear maps,

$$
A=\Omega^{0} \stackrel{d}{\rightarrow} \Omega^{1} \stackrel{d}{\rightarrow} \Omega^{2} \stackrel{d}{\rightarrow} \cdots .
$$

We analyze conditions under which it is possible to define a product as in (5) with properties similar to the properties found by Meyer in the $\mathcal{C}^{\infty}$-case. We prove that the conditions hold for a wide variety of systems, including the above algebraic system when $A$ is smooth over $k$. And we prove additional properties which are new, even in the $\mathcal{C}^{\infty}$-case considered by Meyer. In particular, when the conditions hold, we prove that for any $n \geq 1$ there is a natural exact sequence of $A$-linear maps,

$$
0 \rightarrow \Omega^{1} \otimes_{A} \Omega^{n} \stackrel{i}{\rightarrow} \Omega^{n+1} \stackrel{r}{\rightarrow} \Omega^{n} \rightarrow 0 .
$$

The map $i$ is the product (5) for $p=1$. The differential $d$ is a $k$-linear section of the $A$-linear map $r$ and, in addition, there is a natural $k$-linear retraction $s$ for the $A$-linear inclusion $i$. We prove that the product (5) exists and that it extends to a product,

$$
\Omega^{p} \otimes_{k} C_{n} \rightarrow C_{n+p} \quad \text { denoted } \omega \otimes F \mapsto \omega . F .
$$

In fact, we prove that for any given element $\varepsilon$ in $k$ there are a product (5), depending on $\varepsilon$, and an extension (12) such that, with respect to the products, we have that $\Omega$ is an algebra, $C$ an $\Omega$-module and $d$ is an $\varepsilon$-derivation with respect to the product, that is, for $\omega \in \Omega^{p}$ and $F \in C$ the following equation holds:

$$
d(\omega \cdot F)=d \omega \cdot F+\varepsilon^{p} \omega \cdot d \pi .
$$

In addition, we establish an explicit formula for the product.

One of the main contributions of our work is the construction of Leibniz and Kähler differentials, and the description of their multiplicative structures. Leibniz differentials are universal objects for higher order differentials and their multiplicative structure induces the multiplicative structure on the higher order differentials in applications. In particular, they give a natural explanation for the multiplication on higher order differentials on differential manifolds observed by Meyer. In fact, the $\varepsilon$-calculus explained above gives an important and useful extension of the case $\varepsilon=1$ treated by Meyer. Kähler differentials are universal objects having an additional property explained below, that is shared with the most interesting examples.

The advantage of approaching higher order differentials via universal objects is that the treatment of higher order differentials becomes coordinate free and global, and attains such a generality that it encompasses applications in a wide range, from manifolds to schemes. In particular, in the situation of differentiable manifolds, we do not need to define the multiplication in local coordinates, as Meyer does, and we avoid the tedious task, left by Meyer to the reader, of showing that multiplication is well defined and associative. Our theory could be presented for the global case 
with only a change of terminology and notation. To make the presentation more accessible we have however chosen to treat the case of algebras, and have made a comment (Note (5.20)) about the notational changes that are necessary to adapt to more geometric situations.

One of the main innovations of this work is the $\varepsilon$-formalism indicated above. The reward for carrying out the $\varepsilon$-formalism is that we obtain the algebras of symmetric, skew symmetric and alternating differentials as a natural part of our theory, simply by taking quotients of the Leibniz differentials in the cases $\varepsilon= \pm 1$. The calculus of symmetric and alternating differentials is discussed by Meyer, who termed them "forgotten", respectively, "well known", and he obtains these calculi from his theory of higher order differentials for differentiable manifolds using the action of the symmetric, respectively alternating group, on these differentials. The passage from Leibniz differentials to symmetric, skew symmetric and alternating differentials is made in Section 7. It is worth noting that some of our formulas, in the symmetric case, differ from those obtained by Meyer.

To describe our work in more detail, fix a commutative ring $k$ and a $k$-algebra $A$, which is not necessarily commutative. In addition, fix an element $\varepsilon$ in $k$. Consider a general system (3), consisting of $A$-modules $C_{n}$ with $C_{0}=A$ and $k$-linear maps $d: C_{n} \rightarrow C_{n+1}$. Equivalently, the system may be viewed as a pair $(C, d)$, where $C$ is the direct sum of the $C_{n}$ and $d$ is the $k$-linear endomorphism of degree 1 of $C$ whose components are the maps $d: C_{n} \rightarrow C_{n+1}$. Assume that the unit 1 of $A$, as an element of $C_{0}$, is in the kernel of $d$. Denote by $\Lambda(C)$ the smallest $d$-invariant $A$-submodule of $C$ containing the unit 1. Then, clearly, $\Lambda(C)$ is a homogeneous $A$-submodule of $C$. Its elements will be called the differentials of the system. The system $(C, d)$ will be called a derivation system for $A$, if $d$ is a differential operator of order at most 1 . In this case we write $\Omega(C)$ for the submodule of differentials.

The differentials of the system may be defined inductively: the differentials of degree 0 are the elements of $A=C_{0}$, and the differentials of degree $n+1$ are $A$-linear combinations of elements $d \pi$, where $\pi$ is a differential of degree $n$. Equivalently, the differentials of degree $n$ are the sums of elements of the form

$$
a_{n} d\left(a_{n-1} d\left(\cdots a_{1} d\left(a_{0}\right)\right)\right),
$$

for $a_{0}, \ldots, a_{n} \in A$. In other words, if $A\langle t\rangle$ is the polynomial algebra over $A$ in one non-commuting variable $t$ (see Section 1 ), then the differentials of degree $n$ are the elements of $C$ of the form $P(d)(1)$ where $P$ is a polynomial of degree $n$ in $A\langle t\rangle$.

Therefore, it is not surprising that the algebra $A\langle t\rangle$ plays an important role in the theory of higher order differentials. The material necessary to study the properties of $A\langle t\rangle$ is found in Section 1. We develop a theory of Taylor series in $\varepsilon$-twisted polynomial rings, and we give two fundamental, Leibniz type, formulas (Proposition (1.8)). The theory of Section 1 has a wider scope than being a utensil for higher order differentials. Indeed, the $\varepsilon$-formalism and the Leibniz formulas provide, for example, a natural framework for treating Gaussian polynomials (Note (1.11) and Example (1.13)).

In Section 2 we consider a system obtained from the algebra $A\langle t\rangle$ as follows: Let $\Lambda_{A / k}$ be the quotient of $A\langle t\rangle$ modulo the left ideal $A\langle t\rangle t$, with the $k$-linear endomorphism

$$
d_{t}: \Lambda_{A / k} \rightarrow \Lambda_{A / k},
$$


induced by left multiplication by $t$. The quotient $\Lambda_{A / k}$ is a graded $A$-module and the endomorphism $d_{t}$ is homogeneous of degree 1 . In degree 0 we have that $\Lambda_{A / k}^{0}=A$. Hence we have obtained a system $\left(\Lambda_{A / k}, d_{t}\right)$. Clearly, all elements of $\Lambda_{A / k}$ are differentials of the system. The elements of $\Lambda_{A / k}$ are called the Leibniz differentials of the $k$-algebra $A$. In Remark (2.14) we show that the system $\left(\Lambda_{A / k}, d_{t}\right)$ is universal with respect to general systems $(C, d)$ of the form (9).

We show (Proposition (2.7)) that for the Leibniz differentials there is an exact sequence analogous to (11), which is split by $d_{t}$ and a map $s_{t}$ similar to $s$.

A multiplicative structure on the Leibniz differentials is induced as follows: Let $\Delta_{t}^{\varepsilon}$ be the $k$-linear endomorphism of $A\langle t\rangle$ defined on homogeneous polynomials $Q$ by

$$
\Delta_{t}^{\varepsilon}(Q)=t Q-\varepsilon^{\operatorname{deg} Q} Q t
$$

and extended to $A\langle t\rangle$ by linearity. Then $\Delta_{t}^{\varepsilon}$ is an $\varepsilon$-derivation. Let $\Lambda_{A / k}^{\varepsilon}$ be the smallest $\Delta_{t}^{\varepsilon}$-invariant $A$-submodule of $A\langle t\rangle$ containing 1 . The main result (Proposition (2.12)) of Section 2 states that $\Lambda_{A / k}^{\varepsilon}$ is a graded $k$-subalgebra of $A\langle t\rangle$. Moreover, the composition of the inclusion $\Lambda_{A / k}^{\varepsilon} \subseteq A\langle t\rangle$ and the quotient map $A\langle t\rangle \rightarrow \Lambda_{A / k}$ is an isomorphism of $A$-modules

$$
\Lambda_{A / k}^{\varepsilon} \stackrel{\sim}{\longrightarrow} \Lambda_{A / k}
$$

Under this isomorphism, the $\varepsilon$-derivation $d_{t}^{\varepsilon}$ of $\Lambda_{A / k}^{\varepsilon}$, induced by $\Delta_{t}^{\varepsilon}$, corresponds to $d_{t}$. As a consequence, from the structure on $\Lambda_{A / k}^{\varepsilon}$ as a $k$-algebra over $A$ we obtain a structure on $\Lambda_{A / k}$ as a $k$-algebra over $A$ in such a way that $d_{t}$ becomes an $\varepsilon$-derivation. In Remark (2.14) we show that the Leibniz differentials with this multiplicative structure form a universal object for graded $k$-algebras over $A$ with $\varepsilon$-derivations.

The system of Leibniz differentials $\Lambda_{A / k}$ is not a derivation system because the endomorphism $d_{t}$ is not a differential operator. In Section 3 we consider a derivation system obtained as the quotient of $\Lambda_{A / k}$ by the smallest $d_{t}$-invariant $A$-submodule of $\Lambda_{A / k}$ containing all elements

$$
d_{t}(b a \pi)-b d_{t}(a \pi)-a d_{t}(b \pi)+a b d_{t}(\pi),
$$

for all $a, b$ in $A$ and $\pi$ in $\Lambda_{A / k}$. The endomorphism $d_{t}$ induces a $k$-linear endomorphism $d$ of the quotient. The quotient is denoted $\Omega_{A / k}$ and its elements are called Kähler differentials. The name is justified by the fact that when $A$ is commutative, the first graded piece $\Omega_{A / k}^{1}$ of $\Omega_{A / k}$ is the usual $A$-module of first order Kähler differentials associated to the $k$-algebra $A$ and $d: A \rightarrow \Omega_{A / k}^{1}$ is the usual universal derivation from $A$.

The system of Kähler differentials form a derivation system, that is, the endomorphism $d$ of $\Omega_{A / k}$ is a differential operator of order at most 1 . Moreover, we observe in Remark (3.11) that the system of Kähler differentials is the universal derivation system for $A$. We show (Proposition (3.9)) that for the Kähler differentials there is an exact sequence analogous to (11).

For the given element $\varepsilon$ of $k$, the corresponding product on $\Lambda_{A / k}$ induces a product on $\Omega_{A / k}$. Moreover, with this product, the $A$-module $\Omega_{A / k}$ is a $k$-algebra over $A$, denoted $\Omega_{A / k}^{\varepsilon}$, and the endomorphism $d$ is an $\varepsilon$-derivation. Moreover, we show that the algebra $\Omega_{A / k}^{\varepsilon}$ is universal with respect to graded $k$-algebras over $A$ with $\varepsilon$-derivations $D$ satisfying $D(b 1) a=a D(b 1)$, for all elements $a$ and $b$ of $A$. 
Finally, when $\Omega_{A / k}^{1}$ is a free $A$-module generated by elements $d x_{1}, \ldots, d x_{m}$, we show that each $A$-module $\Omega_{A / k}^{n}$ is free, and we give explicit bases for this module (Proposition (3.12) and Corollary (3.13)). We also extend the theory of Kähler differentials to the case of $A$-modules and show that connections on a module correspond to derivations on the corresponding Kähler differentials (Note (3.15) and Note (3.16)). It is interesting to note that, when $A$ is commutative, the Kähler differentials can be obtained by iterated first order principal parts (Example (5.5)).

The central theme in this work is the problem of deciding, for a given system $(C, d)$, if the module of differentials $\Lambda(C)$ have a multiplicative structure such that the endomorphism $d$ is an $\varepsilon$-derivation. This theme is the subject of Section 4 where we consider, more generally, a triple $(C, \varphi, u)$, where $C$ is an $A$ module and $\varphi$ is a $k$-linear endomorphism of $C$, and $u$ is an element in the kernel of $\varphi$. Assume for simplicity that $C$ is graded, that $\varphi$ is homogeneous of degree 1 , and that $u$ is homogeneous of degree 0 . Via the given endomorphism $\varphi$, we have that $C$ is a module over the polynomial algebra $A\langle t\rangle$. In particular, we have that $C$ is a module over the subalgebra $\Lambda_{A / k}^{\varepsilon}$. Denote by $\Lambda^{\varepsilon}(\varphi)$ the image of the corresponding representation $\Lambda_{A / k}^{\varepsilon} \rightarrow \operatorname{End}_{k}(C)$. Then $C$ is a faithful module over $\Lambda^{\varepsilon}(\varphi)$. Let $\Lambda(C, \varphi, u)$ be the smallest $\varphi$-invariant $A$-submodule of $C$ containing $u$. Then, since $u$ is in the kernel of $\varphi$, it follows from our Leibniz formulas that the evaluation map, $P \mapsto P(\varphi)(u)$, induces a surjection

$$
\Lambda^{\varepsilon}(\varphi) \rightarrow \Lambda(C, \varphi, u)
$$

The main result (Proposition (4.4)) of Section 4 gives criteria for the surjection to be an isomorphism, and, when the criteria are fulfilled, it describes explicitly the induced multiplicative structure on $\Lambda(C, \varphi, C)$. In Section 5 we show that these criteria are fulfilled in all interesting examples. We also show (Lemma (4.2)) that the multiplicative structure is induced from the multiplicative structure of the Kähler differentials if and only if $\varphi$ is a differential operator of order at most 1 .

To describe the contents of the remaining sections 5,6 , and 7 , assume that $A$ is commutative. Let $(C, d)$ be a derivation system. Since $d$ is a differential operator of order at most 1, it follows from the results of Section 3 that there is an $A$-linear map $\Omega_{A / k}^{1} \otimes_{A} C \rightarrow C$, denoted $\rho \otimes_{A} F \mapsto \rho . F$, such that $d_{A / k} f . F=d(f F)-f d F$, for all $f \in A$ and $F \in C$. Moreover, since in addition $d(1)=0$, we have that $d: A \rightarrow C_{1}$ is an $A$-module derivation and we obtain a surjective $A$-linear map $\Omega_{A / k}^{1} \rightarrow \Omega^{1}(C)$. To apply the results of Section 4, we must have that the map $\Omega_{A / k}^{1} \otimes_{A} C \rightarrow C$ factors over the quotient map $\Omega_{A / k}^{1} \otimes_{A} C \rightarrow \Omega^{1}(C) \otimes_{A} C$. When the latter property is satisfied we call the derivation system $(C, d)$ a Kähler system.

Obviously, the system of Kähler differentials is a derivation system. The major part of Section 5 is devoted to other examples of Kähler systems $(C, d)$. In Example (5.7) we introduce the differentials defined by Iitaka, mentioned above. A noncommutative version, which is easier to define, is introduced in Example (5.6). An important generalization of this construction is given in Example (5.9). The generalization may be seen as the affine analogue of the Semple bundle alluded to above. For the constructions in (5.9), we need some properties of first order Kähler differentials of symmetric powers, collected in (5.8). Finally, the original example of Meyer on differentiable manifolds is given in (5.10). All the systems given in the examples are Kähler systems. 
To apply the main result of Section 4, we need an additional property of the system. We say that the Kähler system $(C, d)$ has a Kähler basis if there are elements $x_{1}, \ldots, x_{m}$ of $A=C_{0}$ such that the subset $\mathcal{X}_{1}:=\left\{d x_{1}, \ldots, d x_{m}\right\}$ is an $A$-basis for $\Omega^{1}(C)$, and such that the subset $\mathcal{X}_{n}$ of $C_{n}$ for $n=2,3, \ldots$, defined inductively by

$$
\mathcal{X}_{n+1}=\left\{d \xi \mid \xi \in \mathcal{X}_{n}\right\} \cup\left\{d x_{j} . \xi \mid j=1, \ldots, m, \xi \in \mathcal{X}_{n}\right\},
$$

is an $A$-basis for the $n$ 'th graded piece $\Omega^{n}(C)$ of $\Omega(C)$. For example, for the system $\left(\Omega_{A / k}, d\right)$ of Kähler differentials, a subset $x_{1}, \ldots, x_{m}$ of $A$ is a Kähler basis if and only if the differentials $d x_{j}$ form an $A$-basis for $\Omega_{A / k}^{1}$.

Section 6 contains our results on Kähler systems having a Kähler basis. Let $(C, d)$ be a Kähler system with a Kähler basis. We prove in Theorem (6.7), which is the main result of this article, that the graded pieces $\Omega^{n}(C)$ of the module of differentials fit into an exact sequence analogous to (11). In addition, for the given $\varepsilon$ in $k$ there are products (5) and (12), depending on $\varepsilon$, such that $\Omega(C)$ is a graded $k$-algebra over $A$, such that $C$ is a graded $\Omega(C)$-module, and such that $d$ is an $\varepsilon$-derivation.

In Example (6.9) we note in particular that if $x_{1}, \ldots, x_{m}$ are elements in $A$ such that the differentials $d x_{j}$ form an $A$-basis for $\Omega_{A / k}^{1}$, then $\left\{x_{1}, \ldots, x_{m}\right\}$ is a Kähler basis for the non-commutative version of Iitaka's differentials. Similarly, we observe in Example (6.10) that if $x_{1}, \ldots, x_{m}$ are the coordinates of an open subset $X$ of $\mathbf{R}^{m}$, then $\left\{x_{1}, \ldots, x_{m}\right\}$ is a Kähler basis for the system considered by Meyer. In particular, the conclusions of Theorem (6.7) hold for these systems. At the end of the section we sketch a globalization of the theory.

In the final section we construct the algebras of symmetric, skew symmetric and alternating higher order differentials. As mentioned above it is in this construction that the elegance and usefulness of the $\varepsilon$-calculus can most easily be seen. Assume that $\varepsilon= \pm 1$. The algebra of $\varepsilon$-symmetric Kähler differentials $\Omega_{A / k}^{\varepsilon \text {-sym }}$ is the quotient of the module of Kähler differentials by the ideal of $\varepsilon$-commutators, see Definition (7.6). For $\varepsilon=1$, the algebra is the algebra $\Omega_{A / k}^{\mathrm{sym}}$ of symmetric differentials and for $\varepsilon=-1$ it is the algebra $\Omega_{A / k}^{\text {skew }}$ of skew symmetric differentials. The algebra $\Omega_{A / k}^{\text {alt }}$ is the quotient of $\Omega_{A / k}^{\text {skew }}$ by the ideal generated by squares of elements of odd degree.

We note in (7.6) that these algebras, in addition to the derivation of degree 1 induced by $d$, have a canonical derivation $\delta$ of degree -1 with several interesting properties.

More generally, for any Kähler system $(C, d)$ we define a system of $\varepsilon$-symmetric differentials $\Omega^{\varepsilon \text {-sym }}(C)$ and a system $\Omega^{\text {alt }}(C)$ of alternating differentials. When $(C, d)$ have a Kähler basis, these systems have multiplicative structures, obtained from the algebra $\Omega(C)$ with its $\varepsilon$-product by dividing by suitable ideals. Assume that $\left\{x_{1}, \ldots, x_{m}\right\}$ is a Kähler basis for $(C, d)$. We prove in Proposition (7.7) that the algebra $\Omega^{\mathrm{sym}}(C)$ of symmetric differentials is the commutative $A$-algebra freely generated by the infinitely many differentials $d^{n} x_{j}$ for $n \geq 1$ and $j=1, \ldots, m$ and that $\Omega^{\text {alt }}(C)$ is the alternating $A$-algebra freely generated by the same differentials.

In (7.9) we prove, for any commutative $k$-algebra $A$, that the exterior algebra $\bigwedge \Omega_{A / k}^{1}$ with its exterior differentiation is the quotient of the algebra $\Omega_{A / k}^{\text {alt }}$ of alternating Kähler differentials by the ideal generated by all differentials $d^{2} \omega$.

Finally, in (7.10) we note that the algebra $\Omega_{A / k}^{\mathrm{sym}}$ of symmetric Kähler differentials is equal to the algebra obtained by Iitaka's original construction. 


\section{FORMAL TAYLOR EXPANSIONS}

Formal Taylor expansions provide the basic technique for studying the multiplicative structure of higher order differentials. In this section we introduce Taylor expansions in $\varepsilon$-twisted, non-commutative, polynomial algebras and prove two fundamental Leibniz type formulas for actions on modules. These formulas are central in our treatment of the multiplicative structure of higher order differentials.

We first define the non-commutative polynomial $k$-algebra $A\langle t\rangle$ over $A$, mentioned in the introduction, and study its properties. For every element $\varepsilon$ of $k$ we introduce $\varepsilon$-derivations on graded $k$-algebras over $A$. In particular, we study the inner $\varepsilon$-derivation $\Delta_{t}^{\varepsilon}$ on $A\langle t\rangle$ defined by $\Delta_{t}^{\varepsilon}(Q)=t Q-\varepsilon^{\operatorname{deg} Q} Q t$ on homogeneous elements $Q$ of $A\langle t\rangle$, and extended by linearity. Moreover, given a graded $k$-algebra $B$ over $A$, we study the $\varepsilon$-twisted polynomial algebra $B_{\varepsilon}[h]$, that is the algebra which, as an $A$-module, is freely generated by the powers $h^{i}$, for $i=0,1, \ldots$, and whose multiplication is determined by $h b=\varepsilon^{\operatorname{deg} b} b h$, for all homogeneous elements $b$ of $B$. We study, in particular, Taylor series in the $\varepsilon$-twisted polynomial algebra $A\langle t\rangle_{\varepsilon}[h]$. The main result of this section is the two Leibniz type formulas of Corollary (1.9).

It is noteworthy that the Taylor expansion $(t+h)^{n}=\sum_{i=0}^{\infty}\left(\begin{array}{c}n \\ i\end{array}\right)_{\varepsilon} t^{n-i} h^{i}$ in $k[t]_{\varepsilon}[h]$ defines the Gaussian polynomials $\left(\begin{array}{c}n \\ i\end{array}\right)_{\varepsilon}=\frac{\left(1-\varepsilon^{n}\right)\left(1-\varepsilon^{n-1}\right) \cdots\left(1-\varepsilon^{n-i+1}\right)}{\left(1-\varepsilon^{i}\right)\left(1-\varepsilon^{i-1}\right) \cdots(1-\varepsilon)}$, for $i=0,1, \ldots$. We indicate how the methods used in this section provide a convenient tool for studying Gaussian polynomials.

1.1. Setup. We shall work over a fixed commutative ring $k$. In addition, we fix an element $\varepsilon$ of $k$. Graded modules are always assumed to be $\mathbf{N}$-graded. As a consequence, the element $\varepsilon$ induces in any graded $k$-module a homogeneous $k$ endomorphism which in degree $n$ is multiplication by $\varepsilon^{n}$. The endomorphism will be denoted $x \mapsto \varepsilon_{\text {gr }}(x)$. Clearly, in a graded $k$-algebra, the endomorphism $\varepsilon_{\text {gr }}$ is an endomorphism of algebras.

A $k$-algebra is always assumed to be associative and unitary, but not necessarily commutative. Let $A$ be a fixed $k$-algebra. Unless otherwise specified, an $A$-module is a left $A$-module. An $A$-A-module $N$ is a $k$-module $N$ with a left action and a right action of $A$, both extending the given action of $k$, such that the two actions commute, that is,

$$
a(x b)=(a x) b \quad \text { for } a, b \in A, \quad x \in N .
$$

In other words, an $A$ - $A$-module is a module over the $k$-algebra $A \otimes_{k} A^{\text {op }}$, where $A^{\text {op }}$ is the opposite algebra of $A$.

A $k$-algebra $B$ is said to be a $k$-algebra over $A$ if there is given a homomorphism of $k$-algebras $A \rightarrow B$, called the structure map. Homomorphisms of $k$-algebras over $A$ are assumed to commute with the structure maps. Via the structure map, a $k$-algebra $B$ over $A$ is an $A$ - $A$-module. In particular, a $k$-algebra $B$ over $A$ is an $A$-module and the multiplication $B \otimes_{k} B \rightarrow B$ is $A$-linear when the tensor product is given the structure of an $A$-module inherited from the first factor. Conversely, if a $k$-algebra $B$ has the structure of an $A$-module extending its structure as a $k$-module and such that the multiplication $B \otimes_{k} B \rightarrow B$ is $A$-linear, then $B$ is a $k$-algebra over $A$ with $a \mapsto a 1_{B}$ as structure map.

If $M$ is an $A$-module and $a$ is an element of $A$, we denote by $a_{M}$ the $k$-linear endomorphism of $M$ given by left multiplication by $a$. Clearly, the $\operatorname{ring} \operatorname{End}_{k}(M)$ 
of $k$-linear endomorphisms of $M$ is a $k$-algebra over $A$ with the map $a \mapsto a_{M}$ as structure map.

If $T$ is a subset of a $k$-algebra $B$ over $A$, denote by $A\langle T\rangle$ the smallest subring of $B$ containing $T$ and the image of $A$ in $B$.

1.2. Denote by $A\langle t\rangle$ the polynomial $k$-algebra in one variable over $A$. It is the $k$-algebra over $A$ freely generated by $t$. A more formal definition of the polynomial algebra is obtained as follows:

First, for any $A$ - $A$-module $N$, define

$$
T_{A}^{n}(N):=N \otimes_{A} N \otimes_{A} \cdots \otimes_{A} N,
$$

with $n$ factors in the tensor product. The tensor product is an $A$-A-module, with the left action of $A$ inherited from the first factor and the right action inherited from the last. By definition, $T_{A}^{0}(N):=A$. The direct sum

$$
T_{A}(N):=T_{A}^{0}(N) \oplus T_{A}^{1}(N) \oplus \cdots
$$

is a graded $k$-algebra with the product given by the tensor product under the isomorphism

$$
T_{A}^{p}(N) \otimes_{A} T_{A}^{q}(N)=T_{A}^{p+q}(N) .
$$

The graded $k$-algebra $T_{A}(N)$ contains $A$ as its subring of elements of degree 0 . In particular, $T_{A}(N)$ is a $k$-algebra over $A$. Moreover, the $k$-algebra $T_{A}(N)$ has the following universal property: For any $k$-algebra $B$ over $A$, there is a natural bijection from the set of $A$-A-linear maps $N \rightarrow B$ to the set of homomorphisms $T_{A}(N) \rightarrow B$ of $k$-algebras over $A$.

1.3. Any $A$-module $M$ has a natural extension to an $A$-A-module $M \otimes_{k} A$. Note the following isomorphism,

$$
T_{A}^{n}\left(M \otimes_{k} A\right)=M \otimes_{k} M \otimes_{k} \cdots \otimes_{k} M \otimes_{k} A,
$$

with $M$ occurring $n$ times on the right. Under the latter isomorphism, the multiplication in $T_{A}\left(M \otimes_{k} A\right)$ is given by the formula

$$
\left(x_{p} \otimes \cdots \otimes x_{1} \otimes a\right)\left(y_{q} \otimes \cdots \otimes y_{1} \otimes b\right)=x_{p} \otimes \cdots \otimes x_{1} \otimes a y_{q} \otimes \cdots \otimes y_{1} \otimes b .
$$

By the universal property of (1.2), for any $k$-algebra $B$ over $A$, there is a natural bijection from the set of A-linear maps $M \rightarrow B$ to the set of homomorphisms $T_{A}\left(M \otimes_{k} A\right) \rightarrow B$ of $k$-algebras over $A$.

1.4. Consider in particular the $k$-algebra $T_{A}\left(A \otimes_{k} A\right)$. Its degree- $n$ part is given by

$$
T_{A}^{n}\left(A \otimes_{k} A\right)=A \otimes_{k} A \otimes_{k} \cdots \otimes_{k} A,
$$

with $n+1$ factors on the right. The product is given by the formula in (4.3). Set

$$
t:=1 \otimes 1 \in T_{A}^{1}\left(A \otimes_{k} A\right) .
$$

Then, by the definition of the product, we have that

$$
a_{n} \otimes \cdots \otimes a_{2} \otimes a_{1} \otimes a=a_{n} t \cdots t a_{2} t a_{1} t a .
$$

In particular, $T_{A}\left(A \otimes_{k} A\right)=A\langle t\rangle$. By the universal property of (1.3), for any $k$-algebra $B$ over $A$, there is a bijection from the set of elements e of $B$ to the set of homomorphisms $A\langle t\rangle \rightarrow B$ of k-algebras over $A$. 
The $k$-algebra $A\langle t\rangle$ will be called the $k$-algebra of polynomials over $A$ in the non-central variable $t$. If $B$ is a $k$-algebra over $A$, and $e$ is an element of $B$, then the corresponding map $A\langle t\rangle \rightarrow B$ is fully determined by $t \mapsto e$. The map is the substitution of $e$ in polynomials, denoted

$$
P \mapsto P(e) .
$$

Clearly, the image of the map $A\langle t\rangle \rightarrow B$ is the subalgebra $A\langle e\rangle$ of $B$.

1.5. In a graded $k$-algebra, the $\varepsilon$-commutator $[P, Q]_{\varepsilon}$ of elements $P$ and $Q$ is defined, when $P$ and $Q$ are homogeneous of degrees $p$ and $q$, by the formula

$$
[P, Q]_{\varepsilon}=P Q-\varepsilon^{p q} Q P .
$$

The definition is extended additively to general $P$ and $Q$. In particular, if $P$ is homogeneous of degree $p$, then, for all $Q$,

$$
[P, Q]_{\varepsilon}=P Q-\varepsilon_{\mathrm{gr}}^{p}(Q) P .
$$

1.6. Definition. Let $N$ be an $A$-A-module. Then a $k$-linear map $D: A \rightarrow N$ is called a module derivation if, for all $a, b \in A$,

$$
D(a b)=(D a) b+a(D b) .
$$

In the sequel we need two related notions of derivations. Let $\Gamma$ and $M$ be $A$-modules. Then $\Gamma$ is said to act on $M$ if there is given a $k$-linear map $\mu: \Gamma \otimes_{k} M \rightarrow M$. The map $\mu$ is called the product, and we write $\gamma \cdot x$ or simply $\gamma x$ for $\mu\left(\gamma \otimes_{k} x\right)$. The action is said to be $A$-linear if $\mu$ is $A$-linear when the tensor product $\Gamma \otimes_{k} M$ is considered as an $A$-module via the first factor. An $A$-linear action corresponds to an $A$-linear representation $\Gamma \rightarrow \operatorname{End}_{k}(M)$, denoted $\gamma \mapsto \gamma_{M}$ and defined by $\gamma_{M}(x)=\gamma \cdot x$.

If an action of $\Gamma$ on $M$ is given, a triple $(\alpha, \Delta, D)$ consisting of $k$-linear endomorphisms $\alpha, \Delta: \Gamma \rightarrow \Gamma$ and a $k$-linear endomorphism $D: M \rightarrow M$ will be called a general derivation if, for all $\gamma \in \Gamma$ and $x \in M$, the following equation holds:

$$
D(\gamma \cdot x)=(\Delta \gamma) \cdot x+(\alpha \gamma) \cdot(D x) .
$$

The triple will be called an $\varepsilon$-derivation if, in addition, $\alpha \Delta=\varepsilon \Delta \alpha$.

Let $\Gamma$ be a graded $A$-module and let $\alpha:=\varepsilon_{\mathrm{gr}}$. If (1.6.1) hold and, in addition, $\varepsilon_{\mathrm{gr}} \Delta=\varepsilon \Delta \varepsilon_{\mathrm{gr}}$, then the pair $(\Delta, D)$ will be called an $\varepsilon$-derivation. Note that the condition $\varepsilon_{\mathrm{gr}} \Delta=\varepsilon \Delta \varepsilon_{\mathrm{gr}}$ holds, if $\Delta$ is homogeneous of degree 1 , or if $\varepsilon=1$. Naturally, if $M$ is a graded $A$-module and $\Gamma$ is a homogeneous submodule and if $\Delta$ is the restriction of $D$ to $\Gamma$, then $D$ is called an $\varepsilon$-derivation with respect to the given action, if $(\Delta, D)$ is an $\varepsilon$-derivation.

For instance, assume that $\Gamma=M$ is a graded $k$-algebra over $A$ and consider the action of $\Gamma$ on $M$ given by multiplication in the algebra. Let $P$ be homogeneous of degree $p$ in $\Gamma$. Then, with the $\varepsilon$-commutator

$$
\Delta_{P}^{\varepsilon}(Q):=[P, Q]_{\varepsilon}=P Q-\varepsilon_{\mathrm{gr}}^{p}(Q) P,
$$

the triple $\left(\varepsilon_{\mathrm{gr}}^{p}, \Delta_{P}^{\varepsilon}, \Delta_{P}^{\varepsilon}\right)$ is a derivation, and in fact an $\varepsilon^{p^{2}}$-derivation. Hence, if $P$ is of degree 1 , then $\Delta_{P}^{\varepsilon}$ is an $\varepsilon$-derivation of $\Gamma$. It is called the interior $\varepsilon$-derivation induced by $P$.

In particular, in the polynomial algebra $A\langle t\rangle$, the inner derivation $\Delta_{t}^{\varepsilon}$ is an $\varepsilon$ derivation of $A\langle t\rangle$. It is homogeneous of degree 1 . In particular, the part in degree 0 is a module derivation $A \rightarrow A\langle t\rangle^{1}$, independent of $\varepsilon$. It is the map $a \mapsto t a-a t$, and we denote it $\Delta_{t}$. 
Occasionally, when an action of $\Gamma$ on $M$ is given, we will meet the equation

$$
D(\gamma \cdot x)=(\Delta \gamma) \cdot \beta x+\gamma \cdot(D x),
$$

where $(\Delta, D)$ is as above and $\beta$ is a $k$-linear endomorphism of $M$. If the equation holds and, in addition, $D \beta=\varepsilon \beta D$, then $(\Delta, D, \beta)$ will be called a right $\varepsilon$-derivation. If $M$ is a graded $A$-module, then we may consider (1.6.1) for $\beta:=\varepsilon_{\text {gr }}$. If it holds and, in addition $D \varepsilon_{\mathrm{gr}}=\varepsilon \varepsilon_{\mathrm{gr}} D$, then $(\Delta, D)$ will be called a right $\varepsilon$-derivation. Note that the condition $D \varepsilon_{\mathrm{gr}}=\varepsilon \varepsilon_{\mathrm{gr}} D$ holds, if $D$ is homogeneous of degree -1 , or if $\varepsilon=1$.

1.7. Definition. For any graded $k$-algebra $B$ over $A$, denote by $B_{\varepsilon}[h]$ the $\varepsilon$-twisted polynomial algebra. As a left $B$-module, it is freely generated by the powers $h^{i}$ for $i=0,1, \ldots$. Multiplication in $B_{\varepsilon}[h]$ is given by the rule:

$$
h b=\varepsilon_{\mathrm{gr}}(b) h .
$$

The algebra $B_{\varepsilon}[h]$ is graded by the total degree; the element $h$ is of degree 1 . The algebra $B_{\varepsilon}[h]$ contains $B$ as a graded subalgebra.

Consider in particular the algebra $A\langle t\rangle_{\varepsilon}[h]$. Multiplication in the algebra is determined by the rule that $h$ commutes with the elements of $A$ and $h t=\varepsilon t h$. Denote by $\partial^{\varepsilon}: A\langle t\rangle \rightarrow A\langle t\rangle_{\varepsilon}[h]$ the homomorphism of $k$-algebras over $A$ given by substitution for $t$ of the element $t+h$ of $A\langle t\rangle_{\varepsilon}[h]$, that is, $\partial^{\varepsilon} P=P(t+h)$. Define a family of maps $\partial_{i}^{\varepsilon}: A\langle t\rangle \rightarrow A\langle t\rangle$ for $i=0,1, \ldots$ by the formula,

$$
P(t+h)=\sum_{i=0}^{\infty}\left(\partial_{i}^{\varepsilon} P\right) h^{i} .
$$

Obviously, the map $\partial_{i}^{\varepsilon}$ is an $A$-A-linear endomorphism of $A\langle t\rangle$, and homogeneous of degree $-i$. The endomorphism $\partial_{0}^{\varepsilon}$ is the identity map. Since $\partial^{\varepsilon}$ is a homomorphism of algebras, we have that $\partial^{\varepsilon}(P Q)=\partial^{\varepsilon}(P) \partial^{\varepsilon}(Q)$, that is,

$$
\partial_{n}^{\varepsilon}(P Q)=\sum_{i+j=n}\left(\partial_{i}^{\varepsilon} P\right) \varepsilon_{\mathrm{gr}}^{i}\left(\partial_{j}^{\varepsilon} Q\right) .
$$

Clearly, $\partial_{1}^{\varepsilon} t=1$ and $\partial_{j}^{\varepsilon} t=0$ for $j>1$. It follows from (1.7.2) that $\partial_{1}^{\varepsilon}$ is a right $\varepsilon$-derivation of $A\langle t\rangle$.

1.8. Proposition. Let $B$ be a k-algebra over A. Assume for elements $e, f \in B$ that

$$
f e=\varepsilon e f \quad \text { and } \quad \text { af }=\text { fa for all } a \in A .
$$

Then the following two formulas hold for $P \in A\langle t\rangle$ :

$$
\begin{gathered}
P(e+f)=\sum_{i=0}^{\infty} \partial_{i}^{\varepsilon} P(e) f^{i}, \\
P(e)=\sum_{i=0}^{\infty}(-1)^{i} \varepsilon^{i(i-1) / 2} \partial_{i}^{\varepsilon} P(e+f) f^{i} .
\end{gathered}
$$

Proof. Clearly, given the conditions on $e$ and $f$, there is a unique homomorphism of $k$-algebras over $A$ :

$$
A\langle t\rangle_{\varepsilon}[h] \rightarrow B
$$


such that $t$ is mapped to $e$ and $h$ is mapped to $f$. Obviously, when the homomorphism is applied to the two sides of Equation (1.7.1), the result is Equation (1.8.1).

To prove (1.8.2), note first that the special case, for $B:=A\langle t\rangle_{\varepsilon}[h], e:=t$, and $f:=h$, is the following equation in $A\langle t\rangle_{\varepsilon}[h]$ :

$$
P(t)=\sum_{i=0}^{\infty}(-1)^{i} \varepsilon^{i(i-1) / 2}\left(\partial_{i}^{\varepsilon} P\right)(t+h) h^{i} .
$$

As in the proof of (1.8.1), the special equation (1.8.3) implies the general equation (1.8.2). Hence it suffices to prove (1.8.3).

To do so, let $B$ be the ring of $k$-linear endomorphisms of the graded $A$-module $A\langle t\rangle_{\varepsilon}[h]$. Let $e$ and $f$ be the $k$-linear endomorphisms of $A\langle t\rangle_{\varepsilon}[h]$ defined, for $\Psi \in$ $A\langle t\rangle_{\varepsilon}[h]$, by the equations,

$$
e(\Psi):=(t+h) \Psi, \quad f(\Psi):=-\varepsilon_{\mathrm{gr}}(\Psi) h .
$$

Then $e$ and $f$ belongs to $B$. Clearly, $f$ is $A$-linear and $f e=\varepsilon e f$. Hence Equation (1.8.1) holds in the endomorphism ring $B$. Now we evaluate the two sides of Equation (1.8.1) at the element 1 of $A\langle t\rangle_{\varepsilon}[h]$.

To evaluate the right hand side, note that $f\left(h^{j}\right)=-\varepsilon^{j} h^{j+1}$. It follows that

$$
f^{i}(1)=(-1)^{i} 1 \cdot \varepsilon \cdots \varepsilon^{i-1} h^{i}=(-1)^{i} \varepsilon^{i(i-1) / 2} h^{i} .
$$

Clearly, for $Q \in A\langle t\rangle$ and $\Psi \in A\langle t\rangle_{\varepsilon}[h]$, we have that $Q(e)(\Psi)=Q(t+h) \Psi$. It follows that evaluation at 1 of the right hand side of (1.8.1) is the right hand side of (1.8.3).

Therefore, to finish the proof, it suffices to prove that evaluation of the left side of (1.8.1) is the left side of (1.8.3), that is,

$$
P(e+f)(1)=P .
$$

If $Q \in A\langle t\rangle$, then $(e+f)(Q)=(t+h) Q-\varepsilon_{\mathrm{gr}}(Q) h=t Q$, since $h Q=\varepsilon_{\mathrm{gr}}(Q) h$. It follows easily that $P(e+f)(Q)=P Q$. In particular, (1.8.4) holds, and the proof of the proposition is complete.

1.9. Corollary. Assume as in the setup of (1.6) that an A-linear action of $\Gamma$ on $M$ is given and that $(\alpha, \Delta, D)$ is an $\varepsilon$-derivation. Assume in addition that $\alpha$ is $A$-linear. Then, for $P \in A\langle t\rangle, \gamma \in \Gamma$, and $x \in M$, the following two formulas hold:

$$
\begin{gathered}
P(D)(\gamma \cdot x)=\sum_{i=0}^{\infty} \partial_{i}^{\varepsilon} P(\Delta)\left(\alpha^{i} \gamma\right) \cdot D^{i} x \\
P(\Delta)(\gamma) \cdot x=\sum_{i=0}^{\infty}(-1)^{i} \varepsilon^{i(i-1) / 2} \partial_{i}^{\varepsilon} P(D)\left(\alpha^{i} \gamma \cdot D^{i} x\right) .
\end{gathered}
$$

Proof. View the tensor product $\Gamma \otimes_{k} M$ as an $A$-module via the first factor. Then the endomorphism ring $B:=\operatorname{End}_{k}\left(\Gamma \otimes_{k} M\right)$ is a $k$-algebra over $A$. Consider the endomorphisms $e:=\Delta \otimes_{k} 1_{M}$ and $f:=\alpha \otimes_{k} D$. Then $f$ is $A$-linear because $\alpha$ is $A$-linear, and $f e=\varepsilon e f$, because $\alpha \Delta=\varepsilon \Delta \alpha$. Hence the two equations of (1.8) hold. Clearly, for $Q \in A\langle t\rangle$ we have that $Q(e)=Q(\Delta) \otimes_{k} 1_{M}$. In addition we have that 
$f^{i}=\alpha^{i} \otimes_{k} D^{i}$. Thus the two equations of (1.8) are the following:

$$
\begin{gathered}
P(e+f)=\sum_{i=0}^{\infty} \partial_{i}^{\varepsilon} P(\Delta) \alpha^{i} \otimes_{k} D^{i}, \\
P(\Delta) \otimes_{k} 1_{M}=\sum_{i=0}^{\infty}(-1)^{i} \varepsilon^{i(i-1) / 2} \partial_{i}^{\varepsilon} P(e+f)\left(\alpha^{i} \otimes_{k} D^{i}\right) .
\end{gathered}
$$

Compose the two equations with the given action $\mu: \Gamma \otimes_{k} M \rightarrow M$. Since $(\alpha, \Delta, D)$ is a general derivation, we have that $\mu\left(\Delta \otimes_{k} 1_{M}\right)+\mu\left(\alpha \otimes_{k} D\right)=D \mu$, that is, $\mu(e+f)=D \mu$. Since $\mu$ is $A$-linear, it follows, for any polynomial $Q \in A\langle t\rangle$, that

$$
\mu Q(e+f)=Q(D) \mu .
$$

Therefore we obtain the equations,

$$
\begin{gathered}
P(D) \mu=\sum_{i=0}^{\infty} \mu\left(\partial_{i}^{\varepsilon} P(\Delta) \alpha^{i} \otimes_{k} D^{i}\right), \\
\mu\left(P(\Delta) \otimes_{k} 1_{M}\right)=\sum_{i=0}^{\infty}(-1)^{i} \varepsilon^{i(i-1) / 2} \partial_{i}^{\varepsilon} P(D) \mu\left(\alpha^{i} \otimes_{k} D^{i}\right) .
\end{gathered}
$$

Clearly, the latter two equations evaluated at $\gamma \otimes_{k} x$ yield the two equations of the corollary.

1.10. Note. The two equations of Corollary (1.9) will be called the Leibniz formulas. The corresponding formulas for right $\varepsilon$-derivations $(\Delta, D, \beta)$ are the following two equations, when $P \in A\langle t\rangle$ is homogeneous of degree $p$ :

$$
\begin{gathered}
P(D)(\gamma \cdot x)=\sum_{i=0}^{\infty} \partial_{i}^{\varepsilon} P(\Delta)(\gamma) \cdot \beta^{p-i} D^{i} x, \\
P(\Delta)(\gamma) \cdot \beta^{p} x=\sum_{i=0}^{\infty}(-1)^{i} \varepsilon^{i(i-1) / 2} \partial_{i}^{\varepsilon} P(D)\left(\gamma \cdot D^{i} x\right) .
\end{gathered}
$$

The proof is similar to that of (1.9). Let $e$ and $f$ be the endomorphisms $e:=\Delta \otimes_{k} \beta$ and $f:=1 \otimes_{k} D$. Then $f$ is $A$-linear, and $f e=\varepsilon e f$ because $D \beta=\varepsilon \beta D$. Thus the two equations of Proposition (1.8) hold. If $Q \in A\langle t\rangle$ is homogeneous of degree $q$, then $Q(e)=Q(\Delta) \otimes_{k} \beta^{q}$. Moreover, from (1.6.2) it follows that $\mu Q(e+f)=Q(D) \mu$. Hence, as in the proof of (1.9), the two formulas follow.

1.11. Note. Define the $\varepsilon$-binomial coefficients $\left(\begin{array}{c}n \\ i\end{array}\right)_{\varepsilon}$ by the following equation in $k[t]_{\varepsilon}[h]:$

$$
(t+h)^{n}=\sum_{i=0}^{\infty}\left(\begin{array}{c}
n \\
i
\end{array}\right)_{\varepsilon} t^{n-i} h^{i}
$$

Equivalently, the coefficients are defined by the following equations in $k[t]$ :

$$
\partial_{i}^{\varepsilon} t^{n}=\left(\begin{array}{c}
n \\
i
\end{array}\right)_{\varepsilon} t^{n-i}
$$

Clearly, $\left(\begin{array}{l}n \\ 0\end{array}\right)_{\varepsilon}=\left(\begin{array}{l}n \\ n\end{array}\right)_{\varepsilon}=1$ and $\left(\begin{array}{l}n \\ i\end{array}\right)_{\varepsilon}=0$ for $i>n$. The interchange of $t$ and $h$ defines an isomorphism of the algebra $k[t]_{\varepsilon}[h]$ onto its opposite algebra. As a consequence, 
we obtain from (1.11.1) the symmetry,

$$
\left(\begin{array}{c}
n \\
i
\end{array}\right)_{\varepsilon}=\left(\begin{array}{c}
n \\
n-i
\end{array}\right)_{\varepsilon}
$$

By expanding $(t+h)^{n+p}=(t+h)^{n}(t+h)^{p}$, we obtain the equation,

$$
\left(\begin{array}{c}
n+p \\
i
\end{array}\right)_{\varepsilon}=\sum_{j+l=i} \varepsilon^{j(p-l)}\left(\begin{array}{l}
n \\
j
\end{array}\right)_{\varepsilon}\left(\begin{array}{l}
p \\
l
\end{array}\right)_{\varepsilon}
$$

In particular, for $i>0$,

$$
\left(\begin{array}{c}
n+1 \\
i
\end{array}\right)_{\varepsilon}=\varepsilon^{i}\left(\begin{array}{l}
n \\
i
\end{array}\right)_{\varepsilon}+\left(\begin{array}{c}
n \\
i-1
\end{array}\right)_{\varepsilon}
$$

By multiplying out the product of the $n$ factors on the left side of (1.11.1), it follows that the coefficient $\left(\begin{array}{l}n \\ i\end{array}\right)_{\varepsilon}$ is a sum of powers of $\varepsilon$. In particular, the coefficient is the evaluation at $\varepsilon$ of a universal polynomial with integer coefficients. It follows from the recursion formulas (1.11.4) that the coefficients are in fact the usual $\varepsilon$-binomial coefficients or Gaussian polynomials. To obtain directly the explicit expressions for the Gaussian polynomials, note first the equations

$$
h\left(t^{j} h^{i}\right) t=\varepsilon^{j} t^{j} h^{i+1} t=\varepsilon^{i+j+1} t\left(t^{j} h^{i}\right) h .
$$

It follows that if $R \in k[t]_{\varepsilon}[h]$ is homogeneous of degree $n$, then $h R t=\varepsilon^{n+1} t R h$. In particular, we have for $n>0$ the equation $h(t+h)^{n-1} t=\varepsilon^{n} t(t+h)^{n-1} h$. As a consequence, we obtain the equation

$$
t(t+h)^{n}-(t+h)^{n} t=\left(1-\varepsilon^{n}\right) t(t+h)^{n-1} h .
$$

Comparison of the coefficients of $t^{n+1-i} h^{i}$ yields the equations,

$$
\left(1-\varepsilon^{i}\right)\left(\begin{array}{c}
n \\
i
\end{array}\right)_{\varepsilon}=\left(1-\varepsilon^{n}\right)\left(\begin{array}{c}
n-1 \\
i-1
\end{array}\right)_{\varepsilon}
$$

Hence we obtain the usual expression for the Gaussian polynomial, for $n \geq i$,

$$
\left(\begin{array}{c}
n \\
i
\end{array}\right)_{\varepsilon}=\frac{\left(1-\varepsilon^{n}\right)\left(1-\varepsilon^{n-1}\right) \cdots\left(1-\varepsilon^{n-i+1}\right)}{\left(1-\varepsilon^{i}\right)\left(1-\varepsilon^{i-1}\right) \cdots(1-\varepsilon)} .
$$

1.12. Note. A formula for the composition $\partial_{i}^{\varepsilon} \partial_{j}^{\varepsilon}$ may be obtained as follows:

Let $B$ be the $\varepsilon$-twisted polynomial algebra over the twisted algebra $A\langle t\rangle_{\varepsilon}[k]$, that is,

$$
B:=\left(A\langle t\rangle_{\varepsilon}[h]\right)_{\varepsilon}[k] .
$$

In $B$ we have the relations $k h=\varepsilon h k$ and $k t=\varepsilon t k$. In particular, with $e:=t+h$ and $f:=k$ we have that $f e=\varepsilon e f$. Hence there is a unique homomorphism $\partial^{\prime}: A\langle t\rangle_{\varepsilon}[h] \rightarrow B$ of $k$-algebras over $A$ such that $t \mapsto t+h$ and $h \mapsto k$. It is determined by the equation

$$
\partial^{\prime}\left(Q h^{i}\right)=Q(t+h) k^{i} .
$$

Similarly, with $e:=t$ and $f:=h+k$ we have that $f e=\varepsilon e f$. Hence there is a unique homomorphism $\partial^{\prime \prime}: A\langle t\rangle_{\varepsilon}[h] \rightarrow B$ of $k$-algebras over $A$ such that $t \mapsto t$ and $h \mapsto h+k$. It is determined by the equation

$$
\partial^{\prime \prime}\left(Q h^{i}\right)=Q(t)(h+k)^{i} .
$$

The two compositions $\partial^{\prime} \partial^{\varepsilon}$ and $\partial^{\prime \prime} \partial^{\varepsilon}$ are homomorphisms $A\langle t\rangle \rightarrow B$ of $k$-algebras over $A$. Clearly, under both compositions, the element $t$ is mapped to $t+h+k$. It 
follows that the two compositions are equal, and in fact equal to the homomorphism $P \mapsto P(t+h+k)$. From the equality $\partial^{\prime} \partial^{\varepsilon} P=\partial^{\prime \prime} \partial^{\varepsilon} P$ we deduce in $B$ the equation

$$
\sum_{j}\left(\partial_{j}^{\varepsilon} P\right)(t+h) k^{j}=\sum_{n}\left(\partial_{n}^{\varepsilon} P\right)(t)(h+k)^{n} .
$$

By comparing the coefficients of $h^{i} k^{j}$, we obtain the desired formula for the composition $\partial_{i}^{\varepsilon} \partial_{j}^{\varepsilon}$ :

$$
\partial_{i}^{\varepsilon} \partial_{j}^{\varepsilon}(P)=\left(\begin{array}{c}
i+j \\
j
\end{array}\right)_{\varepsilon} \partial_{i+j}^{\varepsilon}(P) .
$$

Obviously, we have that

$$
\left(\begin{array}{l}
i \\
1
\end{array}\right)_{\varepsilon}=1+\varepsilon+\cdots+\varepsilon^{i-1}
$$

Hence it follows from (1.12.1) that

$$
\left(\partial_{1}^{\varepsilon}\right)^{n}=\pi_{n}^{\varepsilon} \partial_{n}^{\varepsilon},
$$

where

$$
\pi_{n}^{\varepsilon}=\prod_{i=1}^{n}\left(1+\varepsilon+\cdots+\varepsilon^{i-1}\right) .
$$

Clearly, if $\varepsilon=1$, then $\pi_{n}^{\varepsilon}=n$ !. In particular, if $k$ contains the field of rational numbers, then, for $\varepsilon=1$, we have that $\partial_{n}^{\varepsilon}=(1 / n !)\left(\partial_{1}^{\varepsilon}\right)^{n}$. Similarly, for $\varepsilon=0$, we have that $\pi_{n}^{\varepsilon}=1$, and hence $\partial_{n}^{\varepsilon}=\left(\partial_{1}^{\varepsilon}\right)^{n}$. On the other hand, for $\varepsilon=-1$ we have that $\pi_{2}^{\varepsilon}=0$, and it follows that $\partial_{1}^{\varepsilon} \partial_{1}^{\varepsilon}=0$.

1.13. Example. For any element $x \in k$, the following formulas hold:

$$
\begin{gathered}
x^{n}=\sum_{i=0}^{n}\left(\begin{array}{c}
n \\
i
\end{array}\right)_{\varepsilon}(x-1)(x-\varepsilon) \cdots\left(x-\varepsilon^{i-1}\right), \\
\prod_{i=0}^{n-1}\left(1+\varepsilon^{i} x\right)=\sum_{i=0}^{n} \varepsilon^{i(i-1) / 2}\left(\begin{array}{c}
n \\
i
\end{array}\right)_{\varepsilon} x^{i} .
\end{gathered}
$$

To prove the formulas, let $A:=k$ and let $\Gamma=M$ be the polynomial algebra $k[u]$ with the action given by the product in the ring $k[u]$. For polynomials $p, q$ of $\Gamma$, we have the identity

$$
u p q=\left(u p+x u \varepsilon_{\mathrm{gr}} p\right) q+\left(-x \varepsilon_{\mathrm{gr}} p\right) u q .
$$

Hence, if $D:=u$ is multiplication by $u$ in $\Gamma$, and $\Delta:=u\left(1+x \varepsilon_{\mathrm{gr}}\right)$ and $\alpha:=-x \varepsilon_{\mathrm{gr}}$, then $(\alpha, \Delta, D)$ is an $\varepsilon$-derivation for the action. Clearly, for a constant polynomial $q$ in $k[u]$ we have the identities

$$
\Delta^{i}(q)=(1+x) \cdots\left(1+\varepsilon^{i-1} x\right) u^{i} q, \quad \alpha^{i}(q)=(-1)^{i} x^{i} q .
$$

Therefore, by (1.9) applied with $P:=t^{n}, \gamma:=1$, and $x:=1$, we obtain the two equations

$$
\begin{aligned}
& u^{n}=\sum_{i=0}^{n}\left(\begin{array}{c}
n \\
i
\end{array}\right)_{\varepsilon}(1+x) \cdots\left(1+\varepsilon^{n-i-1} x\right)(-1)^{i} x^{i} u^{n} \\
& (1+x) \cdots\left(1+\varepsilon^{n-1} x\right) u^{n}=\sum_{i=0}^{n} \varepsilon^{i(i-1) / 2}\left(\begin{array}{c}
n \\
i
\end{array}\right)_{\varepsilon} x^{i} u^{n} .
\end{aligned}
$$


Divide by $u^{n}$. Clearly (1.13.2) follows from (1.13.4). By (1.11.3), we obtain from (1.13.3) the equation

$$
1=\sum_{i=0}^{n}\left(\begin{array}{c}
n \\
i
\end{array}\right)_{\varepsilon}(1+x) \cdots\left(1+\varepsilon^{i-1} x\right)(-1)^{n-i} x^{n-i} .
$$

Since $k$ and $x$ are arbitrary, (1.13.1) follows from (1.13.5) after the substitution $x:=-1 / x$.

Note finally, for $n \geq 1$, the following formula in $k[[t]]$ :

$$
\prod_{i=0}^{n-1} \frac{1}{1-\varepsilon^{i} t}=\sum_{j=0}^{\infty}\left(\begin{array}{c}
n+j-1 \\
j
\end{array}\right)_{\varepsilon} t^{j}
$$

To prove (1.13.6), consider the graded completion $B:=\widehat{k[t]_{\varepsilon}[h]}$ of $k[t]_{\varepsilon}[h]$. In $B$ we have the identities

$$
\sum_{m, i=0}^{\infty}\left(\begin{array}{c}
m \\
i
\end{array}\right)_{\varepsilon} t^{m-i} h^{i}=\frac{1}{1-(t+h)}=\sum_{i=0}^{\infty} \frac{1}{(1-t) \cdots\left(1-\varepsilon^{i} t\right)} h^{i} .
$$

Indeed, the first identity holds by (1.11.1). Clearly $h^{i}(1-t-h)=\left(1-\varepsilon^{i} t\right) h^{i}-h^{i+1}$. It follows that the product of $1-t-h$ and the series on the right side of (1.13.7) is equal to 1 . Hence the second identity in (1.13.7) holds. Clearly, the asserted formula (1.13.6) follows by comparing the coefficients to $h^{n-1}$ in (1.13.7).

\section{LEIBNIZ DIFFERENTIALS}

The Leibniz differentials form a universal object for higher order differentials. In this section we define Leibniz differentials and give their main properties. The Leibniz differentials are the elements of the quotient $\Lambda_{A / k}$ of $A\langle t\rangle$ modulo the left ideal $A\langle t\rangle t$. It is a graded $A$-module and comes with the natural $k$-endomorphism $d_{t}$ induced by left multiplication by $t$. Denote the $n$ 'th graded piece of $\Lambda_{A / k}$ by $\Lambda_{A / k}^{n}$. We prove that, for every $n \geq 1$, we have an exact sequence,

$$
0 \rightarrow \Lambda_{A / k}^{1} \otimes_{A} \Lambda_{A / k}^{n} \stackrel{i_{t}}{\longrightarrow} \Lambda_{A / k}^{n+1} \stackrel{r_{t}}{\longrightarrow} \Lambda_{A / k}^{n} \rightarrow 0,
$$

of $A$ - $A$-modules, which is split by right $A$-linear maps.

For every element $\varepsilon$ of $k$ we let $\Lambda_{A / k}^{\varepsilon}$ be the smallest $A$-submodule of $A\langle t\rangle$ which contains 1 and is invariant under the inner $\varepsilon$-derivation $\Delta_{t}^{\varepsilon}$. We show that $\Lambda_{A / k}^{\varepsilon}$ is a $k$-subalgebra of $A\langle t\rangle$ over $A$ and that the composition of the inclusion $\Lambda_{A / k}^{\varepsilon} \rightarrow A\langle t\rangle$ and the quotient map $A\langle t\rangle \rightarrow \Lambda_{A / k}$ is an isomorphism $\Lambda_{A / k}^{\varepsilon} \rightarrow \Lambda_{A / k}$ of $A$-modules, and the $\varepsilon$-derivation $\Delta_{t}^{\varepsilon}$ corresponds to the $k$-endomorphism under the isomorphism. Via this isomorphism the Leibniz differentials inherit, for every element $\varepsilon$ of $k$, a ring structure, such that $d_{t}$ becomes an $\varepsilon$-derivation. We show that the module of Leibniz differentials with this ring structure is a universal object for graded $k$-algebras over $A$ with $\varepsilon$-derivations.

2.1. Lemma. Under the identification of $A\langle t\rangle^{1}$ and $A \otimes_{k} A$, let $I_{A / k} \subseteq A\langle t\rangle^{1}$ be the kernel of the multiplication map $A \otimes_{k} A \rightarrow A$. Then $I_{A / k}$ is an A-A-submodule of $A\langle t\rangle^{1}$. Moreover, there is a decomposition into homogeneous A-submodules,

$$
A\langle t\rangle=A \oplus A\langle t\rangle I_{A / k} \oplus A t,
$$


and the projection onto the second factor, in degree $p \geq 1$, is the map

$$
a_{p} t \cdots t a_{1} t a_{0} \mapsto a_{p} t \cdots t\left(a_{1} t a_{0}-a_{1} a_{0} t\right) .
$$

Proof. The multiplication map $\mu: A \otimes_{k} A \rightarrow A$ is $A$-A-linear. Hence $I_{A / k}$ is an $A$-A-submodule of $A\langle t\rangle^{1}$. Under the multiplication map, a polynomial $P=\sum_{i} b_{i} t a_{i}$ is mapped to $\sum_{i} b_{i} a_{i}$. It follows that $I_{A / k}$ is generated as an $A$-module by the polynomials $t a-a t$ for $a \in A$. In particular, there is a homogeneous $A$-linear map Ev : $A\langle t\rangle \rightarrow A \oplus A\langle t\rangle I_{A / k}$, defined in degree $p \geq 1$ by (2.1.2) and which in degree 0 is the identity of $A$. Clearly, the map Ev vanishes on $A\langle t\rangle t$ and it is the identity on the submodule $A \oplus A\langle t\rangle I_{A / k}$. The assertions of the lemma follow easily.

2.2. Definition. Denote by $\Lambda_{A / k}$ the quotient of $A\langle t\rangle$ modulo the left ideal $A\langle t\rangle t$. As the ideal $A\langle t\rangle t$ is homogeneous, the quotient $\Lambda_{A / k}$ is a graded $A$-module. Denote by

$$
d_{t}: \Lambda_{A / k} \rightarrow \Lambda_{A / k}
$$

the $k$-linear endomorphism induced by left multiplication by $t$. The elements of $\Lambda_{A / k}$ will be called Leibniz differentials. Whenever it is necessary to indicate the dependence on the given $k$-algebra $A$, we will write $d_{A / k, t}$ for $d_{t}$.

In degree 0 , the quotient map $A\langle t\rangle \rightarrow \Lambda_{A / k}$ defines an isomorphism of $A$ onto the Leibniz differentials of degree 0 , and we will always identify $\Lambda_{A / k}^{0}$ and $A$. In particular, the unit 1 of $A$ will be considered as an element of $\Lambda_{A / k}$. Note that, since $d_{t}$ is induced by left multiplication by $t$, the polynomial $P$ is mapped to $P\left(d_{t}\right)(1)$ under the quotient map $A\langle t\rangle \rightarrow \Lambda_{A / k}$.

It follows from (2.1.1) that the canonical map $A\langle t\rangle \rightarrow \Lambda_{A / k}$ restricts to a homogeneous $A$-linear isomorphism,

$$
A \oplus A\langle t\rangle I_{A / k} \stackrel{\sim}{\longrightarrow} \Lambda_{A / k} .
$$

Since $I_{A / k}$ is an $A$-A-submodule of $A\langle t\rangle^{1}$, it follows that the left ideal $A\langle t\rangle I_{A / k}$ is an $A$-A-submodule of $A\langle t\rangle$. Hence the map (2.2.1) induces on $\Lambda_{A / k}$ a canonical structure of an $A$ - $A$-module.

The map (2.2.1) in degree 0 is the identification of $A$ and $\Lambda_{A / k}^{0}$. In degree 1, the map is an $A$-A-isomorphism from the $A$-A-submodule $I_{A / k}$ of $A\langle t\rangle^{1}$ to the $A$-Amodule $\Lambda_{A / k}^{1}$ of Leibniz differentials of degree 1 . Note that, by (2.1), under the isomorphism, the Leibniz differential $d_{t} a$ of $\Lambda_{A / k}^{1}$ corresponds to the polynomial $t a-a t=\Delta_{t} a$ of $I_{A / k}$. We saw in (1.6) that $\Delta_{t}$ is a module derivation on $A\langle t\rangle^{1}$. As a consequence, with respect to the $A$-A-module structure on $\Lambda_{A / k}^{1}$, the map $d_{t}: A \rightarrow \Lambda_{A / k}^{1}$ is a module derivation, that is,

$$
d_{t}(a b)=\left(d_{t} a\right) b+a\left(d_{t} b\right) .
$$

The differentials $d_{t} a$ for $a \in A$ generate $\Lambda_{A / k}^{1}$ as an $A$-module. It follows from (2.2.2) that they also generate $\Lambda_{A / k}^{1}$ as a right $A$-module.

For $n \geq 1$, the differential $d_{t}: \Lambda_{A / k}^{n} \rightarrow \Lambda_{A / k}^{n+1}$ is right $A$-linear, since it corresponds, via the isomorphism (2.2.1), to left multiplication by $t$ in $A\langle t\rangle I_{A / k}$.

For an $A$-module $M$, the tensor product $\Lambda_{A / k}(M):=\Lambda_{A / k} \otimes_{A} M$ is defined using the structure on $\Lambda_{A / k}$ as a right $A$-module, and the tensor product is given 
the structure as a left $A$-module via the structure on $\Lambda_{A / k}$ as a left $A$-module. For $n \geq 1$ the differential induces a $k$-linear map

$$
d_{t, M}=d_{t} \otimes_{A} 1_{M}: \Lambda_{A / k}^{n}(M) \rightarrow \Lambda_{A / k}^{n+1}(M) .
$$

Similarly, if $N$ is a right $A$-module, then $N \otimes_{A} \Lambda_{A / k}^{1}$ is a right $A$-module.

2.3. Lemma. Let $M$ and $M^{\prime}$ be $A$-modules and let $v: M \rightarrow M^{\prime}$ be a $k$-linear map. Then there is an A-linear map

$$
v_{0}: \Lambda_{A / k}^{1} \otimes_{A} M \rightarrow M^{\prime},
$$

determined, for $a \in A$ and $x \in M$, by the equation

$$
v_{0}\left(d_{t} a \otimes_{A} x\right)=v(a x)-a v(x) .
$$

Proof. The equation (2.3.1) determines $v_{0}$, since the differentials $d_{t} a$ generate $\Lambda_{A / k}^{1}$ as an $A$-module. To define $v_{0}$, consider the map

$$
A\langle t\rangle^{1} \otimes_{A} M=A \otimes_{k} A \otimes_{A} M=A \otimes_{k} M \rightarrow M^{\prime},
$$

where $A \otimes_{k} M \rightarrow M^{\prime}$ is the $A$-linear extension of $v$, determined by $a \otimes_{k} x \mapsto a v(x)$. Define $v_{0}$ as the composition of (2.3.2) and the map $\Lambda_{A / k}^{1} \otimes_{A} M \rightarrow A\langle t\rangle^{1} \otimes_{A} M$ induced by the inclusion $\Lambda_{A / k}^{1}=I_{A / k} \subseteq A\langle t\rangle^{1}$ of (2.1). The equation (2.3.1) is easily verified.

2.4. Definition. Given a $k$-linear map $v: M \rightarrow M^{\prime}$. The $A$-linear map $v_{0}$ given in Lemma (2.3) will be denoted by $\rho \otimes_{A} x \mapsto \rho . x$. The particular $A$-linear map $v_{0}$ obtained $M=M^{\prime}=\Lambda_{A / k}$ and $v:=d_{t}$ will be denoted by

$$
i_{t}: \Lambda_{A / k}^{1} \otimes_{A} \Lambda_{A / k} \rightarrow \Lambda_{A / k} .
$$

As the tensor product is over $A$, we have that $(\rho a) . \pi=\rho .(a \pi)$ for $\rho \in \Lambda_{A / k}^{1}, a \in A$ and $\pi \in \Lambda_{A / k}$. Clearly, the map $i_{t}$ is homogeneous of degree 1. By (2.3.1), we have that

$$
d_{t} a . \pi=d_{t}(a \pi)-a d_{t} \pi .
$$

Since the map $d_{t}$ is right $A$-linear, it follows easily that the map $i_{t}$ is $A$-A-linear.

2.5. Lemma. Let $N$ and $N^{\prime}$ be right $A$-modules and let $w: N \rightarrow N^{\prime}$ be a $k$-linear map. Then there is a right A-linear map, homogeneous of degree -1,

$$
w_{t}: N \otimes_{A} \Lambda_{A / k} \rightarrow N^{\prime} \otimes_{A} \Lambda_{A / k},
$$

determined, for $x \in N$, by the equations

$$
\begin{aligned}
& w_{t}\left(x \otimes_{A} d_{t} a\right)=w(x) a-w(x a) \quad \text { for } a \in A . \\
& w_{t}\left(x \otimes_{A} d_{t} \pi\right)=w(x) \otimes_{A} \pi \quad \text { for } \pi \in \Lambda_{A / k}^{n}, n \geq 1 .
\end{aligned}
$$

Moreover, for $\rho \in \Lambda_{A / k}^{1}$ and $\pi \in \Lambda_{A / k}$, we have the equation

$$
w_{t}\left(x \otimes_{A} \rho . \pi\right)=w_{t}\left(x \otimes_{A} \rho\right) \otimes_{A} \pi .
$$

Proof. The part of $w_{t}$ in degree $n$ is required to be a right $A$-linear map,

$$
w_{n}: N \otimes_{A} \Lambda_{A / k}^{n+1} \rightarrow N^{\prime} \otimes_{A} \Lambda_{A / k}^{n} .
$$

Since the differentials $d_{t} \pi$, for $\pi \in \Lambda_{A / k}^{n}$, generate $\Lambda_{A / k}^{n+1}$ as an $A$-module, the maps $w_{n}$ are fully determined by the equations (2.5.1) and (2.5.2). 
For $n=0$, the proof of the existence of the map $w_{n}$ is entirely analogous to the proof of Lemma (2.3).

To prove the remaining assertions, use that $A\langle t\rangle^{n+1}=A \otimes_{k} A\langle t\rangle^{n}$, as follows from (1.4). Under the identification, a polynomial atP of $A\langle t\rangle^{n+1}$ corresponds to $a \otimes_{k} P$. It follows that there is a $k$-linear map $\tilde{w}: N \otimes_{A} A\langle t\rangle \rightarrow N^{\prime} \otimes_{A} A\langle t\rangle$ of degree -1 , determined for $P \in A\langle t\rangle$ by the equation

$$
\tilde{w}\left(x \otimes_{A} t P\right)=w(x) \otimes_{A} P .
$$

Consider a polynomial $Q$ of degree $n+1$ in the ideal $A\langle t\rangle t$. Assume that $n \geq 1$. Then $Q$ is a sum of polynomials of the form atPt. It follows from (2.5.4) that $\tilde{w}\left(x \otimes_{A} Q\right)$ is the sum of terms of the form $w(x a) \otimes_{A} P t$. Therefore, since Pt belongs to $A\langle t\rangle t$, it follows that $\tilde{w}$ for $n \geq 1$ induces a map (2.5.4) of the quotients. Clearly, (2.5.2) follows from (2.5.4). Moreover, since the map $d_{t}$ is right $A$-linear in degree $n \geq 1$, it follows from (2.5.2) that $w_{n}$ is right $A$-linear.

Finally, to prove (2.5.3) we may, since the tensor products are over $A$, assume that $\rho=d_{t} a$ for $a \in A$ and $\pi \in \Lambda_{A / k}^{n}$. If $n=0$, then (2.5.3) is trivial. Assume $n \geq 1$. Since $d_{t} a . \pi=d_{t}(a \pi)-a d_{t} \pi$, it follows from (2.5.2) and (2.5.1) that

$$
w_{t}\left(x \otimes_{A} d_{t} a . \pi\right)=w(x) \otimes_{A} a \pi-w(x a) \otimes_{A} \pi=w_{0}\left(x \otimes_{A} d_{t} a\right) \otimes_{A} \pi .
$$

Thus (2.5.3) holds.

2.6. Definition. The right $A$-linear map of degree -1 obtained from Lemma (2.5) by taking as $w$ the identity map of $A$ will be denoted

$$
r_{t}: \Lambda_{A / k} \rightarrow \Lambda_{A / k}
$$

Since the identity map is $A$-linear, it follows easily that $r_{t}$ is $A$ - $A$-linear. Moreover, since the identity map is right $A$-linear, it follows from (2.5.1) that $r_{0}$ is the zero map. Hence, from (2.5.2) and (2.5.3) we obtain, for $n \geq 1$ and $a \in A, \rho \in \Lambda_{A / k}^{1}$, and $\pi \in \Lambda_{A / k}^{n}$, the two equations

$$
r_{t}\left(a d_{t} \pi\right)=a \pi, \quad r_{t}(\rho . \pi)=0 .
$$

The right $A$-linear map of degree -1 obtained from Lemma (2.5) by taking as $w$ the $k$-linear map $-d_{t}: A \rightarrow \Lambda_{A / k}^{1}$ will be denoted

$$
s_{t}: \Lambda_{A / k} \rightarrow \Lambda_{A / k}^{1} \otimes_{A} \Lambda_{A / k} .
$$

Since $d_{t}(a b)=\left(d_{t} a\right) b+a d_{t} b$, it follows from (2.5.1) that $s_{0}$ is the identity map. Hence, from (2.5.2) and (2.5.3) we obtain, for $n \geq 1$ and $a \in A, \rho \in \Lambda_{A / k}^{1}$, and $\pi \in \Lambda_{A / k}^{n}$, the two equations

$$
s_{t}\left(a d_{t} \pi\right)=-d_{t} a \otimes_{A} \pi, \quad s_{t}(\rho . \pi)=\rho \otimes_{A} \pi .
$$

2.7. Proposition. Assume that $n \geq 1$. Then the following sequence of $A$-A-linear maps is exact:

$$
0 \longrightarrow \Lambda_{A / k}^{1} \otimes_{A} \Lambda_{A / k}^{n} \stackrel{i_{t}}{\longrightarrow} \Lambda_{A / k}^{n+1} \stackrel{r_{t}}{\longrightarrow} \Lambda_{A / k}^{n} \longrightarrow 0,
$$

and split by the right $A$-linear maps $d_{t}$ and $s_{t}$.

Proof. The assertion follows from the equations of (2.6). Indeed, it follows directly that $r_{t} d_{t}=1$ and $s_{t} i_{t}=1$. Hence, to finish the proof, it suffices to prove the equation $i_{t} s_{t}+d_{t} r_{t}=1$. 
By additivity, it suffices to evaluate the equation on an element of the form $a d_{t} \pi$ for $a \in A$ and $\pi \in \Lambda_{A / k}^{n}$. By (2.6.2) and (2.4.2) we have that

$$
i_{t} s_{t}\left(a d_{t} \pi\right)=-d_{t} a . \pi=-d_{t}(a \pi)+a d_{t} \pi .
$$

By (2.6.1), we have that

$$
d_{t} r_{t}\left(a d_{t} \pi\right)=d_{t}(a \pi)
$$

The asserted equation $i_{t} s_{t}+d_{t} r_{t}=1$ follows.

2.8. Definition. By an abuse of notation, denote by $t$ the left multiplication by $t$ in $A\langle t\rangle$. Then the quotient map $A\langle t\rangle \rightarrow \Lambda_{A / k}$ is a surjective homomorphism of $A$-modules with $k$-linear endomorphisms

$$
(A\langle t\rangle, t) \rightarrow\left(\Lambda_{A / k}, d_{t}\right)
$$

More generally, for a given $A$-module $C$, a $k$-linear endomorphism $\varphi$ of $C$ and an element $u$ of $C$, there is a unique homomorphism of $A$-modules with $k$-linear endomorphisms

$$
(A\langle t\rangle, t) \rightarrow(C, \varphi)
$$

under which 1 is mapped to $u$. It is the map $P \mapsto P(\varphi)(u)$, and it is denoted $\operatorname{Ev}_{\varphi, u}$. Clearly, the image in $C$ is the smallest $\varphi$-invariant $A$-submodule containing $u$. The image will be denoted $\Lambda(C, \varphi, u)$. If $\varphi u=0$, then the map $\operatorname{Ev}_{\varphi, u}$ induces a homomorphism

$$
\left(\Lambda_{A / k}, d_{t}\right) \rightarrow(C, \varphi)
$$

2.9. Lemma. Let $\Gamma$ be a graded $k$-algebra over $A$, and let $D$ be an $\varepsilon$-derivation of $\Gamma$. Then the A-submodule $\Lambda(\Gamma, D, 1)$ of $\Gamma$ is a $k$-subalgebra over $A$. In fact, for $P \in A\langle t\rangle$ and $x \in \Gamma$, we have the equation

$$
P(D)(1) x=\sum_{i=0}^{\infty}(-1)^{i} \varepsilon^{i(i-1) / 2} \partial_{i}^{\varepsilon} P(D) D^{i} x .
$$

Proof. The equation follows from (1.9.2) by taking $C:=\Gamma, \alpha=\varepsilon_{\mathrm{gr}}$ and $\gamma=1$. The $A$-submodule $\Lambda(\Gamma, D, 1)$ is $D$-invariant. Hence, if $x$ belongs to $\Lambda(\Gamma, D, 1)$, then the right hand side of (2.9.1) belongs to $\Lambda(\Gamma, D, 1)$. So the product $P(D)(1) x$ belongs to $\Lambda(\Gamma, D, 1)$. Since any element of $\Lambda(\Gamma, D, 1)$ is of the form $Q(D)(1)$ for $Q \in A\langle t\rangle$, it follows that $\Lambda(\Gamma, D, 1)$ is stable under the product in $\Gamma$. Obviously, $\Lambda(\Gamma, D, 1)$ contains the image of the structure map $a \mapsto a 1$. Therefore $\Lambda(\Gamma, D, 1)$ is a $k$-subalgebra over $A$. 
2.10. Lemma. The following four equations hold for polynomials $P, S$, and $R$ in $A\langle t\rangle$ :

$$
\begin{aligned}
P S & =\sum_{i=0}^{\infty} \partial_{i}^{\varepsilon} P\left(\Delta_{t}^{\varepsilon}\right)\left(\varepsilon_{\mathrm{gr}}^{i} S\right) t^{i} . \\
P\left(\Delta_{t}^{\varepsilon}\right)(S) & =\sum_{i=0}^{\infty}(-1)^{i} \varepsilon^{i(i-1) / 2}\left(\partial_{i}^{\varepsilon} P\right) \varepsilon_{\mathrm{gr}}^{i}(S) t^{i} . \\
P\left(\Delta_{t}^{\varepsilon}\right)(S R) & =\sum_{i=0}^{\infty} \partial_{i}^{\varepsilon} P\left(\Delta_{t}^{\varepsilon}\right)\left(\varepsilon_{\mathrm{gr}}^{i} S\right) \Delta_{t}^{\varepsilon i} R . \\
P\left(\Delta_{t}^{\varepsilon}\right)(S) R & =\sum_{i=0}^{\infty}(-1)^{i} \varepsilon^{i(i-1) / 2} \partial_{i}^{\varepsilon} P\left(\Delta_{t}^{\varepsilon}\right)\left(\varepsilon_{\mathrm{gr}}^{i}(S) \Delta_{t}^{\varepsilon i}(R)\right) .
\end{aligned}
$$

Proof. With respect to the product in the algebra $A\langle t\rangle$, the endomorphism $\Delta_{t}^{\varepsilon}$ of (1.6) is an $\varepsilon$-derivation. So the two equations of (1.9), with $\gamma:=S$ and $x:=R$, yield the last two equations.

Again, if we identify $t$ with left multiplication in the algebra $A\langle t\rangle$, then it follows from the equation

$$
t P Q=\left(\Delta_{t}^{\varepsilon} P\right) Q+\left(\varepsilon_{\mathrm{gr}} P\right) t Q
$$

that the pair $\left(\Delta_{t}^{\varepsilon}, t\right)$ is an $\varepsilon$-derivation. So the two equations of (1.9), with $\gamma:=S$ and $x:=1$, yield the first two equations.

2.11. Lemma. The inner $\varepsilon$-derivation $\Delta_{h}^{\varepsilon}$ of $A\langle t\rangle_{\varepsilon}[h]$ vanishes on the subalgebra $A\langle t\rangle$. Moreover, for any polynomial $P$ of $A\langle t\rangle$ we have the equation

$$
\partial^{\varepsilon} \Delta_{t}^{\varepsilon} P=\Delta_{t}^{\varepsilon} \partial^{\varepsilon} P+\Delta_{h}^{\varepsilon} \partial^{\varepsilon} P .
$$

Proof. If $Q \in A\langle t\rangle$, then $\Delta_{h}^{\varepsilon} Q=h Q-\varepsilon_{\mathrm{gr}}(Q) h=0$ by definition of the multiplication in $A\langle t\rangle_{\varepsilon}[h]$. Thus the first assertion holds. Since $\partial^{\varepsilon}$ is a homomorphism of graded algebras and $\partial^{\varepsilon} t=t+h$, it follows that $\partial^{\varepsilon} \Delta_{t}^{\varepsilon}=\Delta_{t+h}^{\varepsilon} \partial^{\varepsilon}$. Clearly $\Delta_{t+h}^{\varepsilon}=\Delta_{t}^{\varepsilon}+\Delta_{h}^{\varepsilon}$. Hence the second assertion holds.

2.12. Proposition. Let $\mathrm{Ev}^{\varepsilon}: A\langle t\rangle \rightarrow A\langle t\rangle$ be the endomorphism defined by the equation $\operatorname{Ev}^{\varepsilon}(P):=P\left(\Delta_{t}^{\varepsilon}\right)(1)$, and denote by $\Lambda_{A / k}^{\varepsilon}$ the image of $\mathrm{Ev}^{\varepsilon}$. Then $\Lambda_{A / k}^{\varepsilon}$ is the smallest $\Delta_{t}^{\varepsilon}$-invariant $A$-submodule containing the unity 1 , and it is a homogeneous k-subalgebra over $A$. Moreover, a polynomial $Q$ belongs to $\Lambda_{A / k}^{\varepsilon}$ if and only if $\partial_{i}^{\varepsilon} Q=0$ for $i>0$. Furthermore, we have the decomposition,

$$
A\langle t\rangle=\Lambda_{A / k}^{\varepsilon} \oplus A\langle t\rangle t,
$$

and the projection on the first factor is the map $\mathrm{Ev}^{\varepsilon}$. In particular, the map $\mathrm{Ev}^{\varepsilon}$ is equal to the identity on $\Lambda_{A / k}^{\varepsilon}$ and the kernel of $\mathrm{Ev}^{\varepsilon}$ is the left ideal of $A\langle t\rangle t$.

Finally, for polynomials $Q \in \Lambda_{A / k}^{\varepsilon}$ and $R \in A\langle t\rangle$, we have the equation

$$
Q R=Q\left(\Delta_{t}^{\varepsilon}\right)(R) .
$$

Proof. The map $\mathrm{Ev}^{\varepsilon}$ is obviously homogeneous, so the image is a homogeneous submodule in $A\langle t\rangle$. In the notation of (2.8), we have that $\Lambda_{A / k}^{\varepsilon}=\Lambda\left(A\langle t\rangle, \Delta_{t}^{\varepsilon}, 1\right)$. Hence the first assertion follows from Lemma (2.9).

Let $\Gamma^{\varepsilon}$ be the set of polynomials $Q \in A\langle t\rangle$ such that $\partial_{i}^{\varepsilon} Q=0$ for $i>0$. If $Q \in \Gamma^{\varepsilon}$, then $\partial^{\varepsilon} Q=Q$. Therefore, it follows from Lemma (2.11) that $\partial^{\varepsilon}\left(\Delta_{t}^{\varepsilon} Q\right)=\Delta_{t}^{\varepsilon} Q$ and 
hence $\Delta_{t}^{\varepsilon} Q$ belongs to $\Gamma^{\varepsilon}$. Thus $\Gamma^{\varepsilon}$ is $\Delta_{t}^{\varepsilon}$-invariant. In addition, $\Gamma^{\varepsilon}$ is an $A$ submodule because the maps $\partial_{i}^{\varepsilon}$ are $A$-linear and, obviously, the unity 1 belongs to $\Gamma^{\varepsilon}$. As $\Lambda_{A / k}^{\varepsilon}$ is the smallest $\Delta_{t}^{\varepsilon}$-invariant $A$-submodule containing 1 , it follows that $\Lambda_{A / k}^{\varepsilon}$ is contained in $\Gamma^{\varepsilon}$. Conversely, if $Q$ belongs to $\Gamma^{\varepsilon}$, then it follows from (2.10.2) with $P:=Q$ and $S:=1$ that

$$
\operatorname{Ev}^{\varepsilon}(Q)=Q
$$

In particular, then $Q$ belongs to the image $\Lambda_{A / k}^{\varepsilon}$. Hence $\Lambda_{A / k}^{\varepsilon}=\Gamma^{\varepsilon}$.

Consider Equation (2.12.1). For any polynomial $P$ we have the equation

$$
P=\operatorname{Ev}^{\varepsilon}(P)+\left(\sum_{i=0}^{\infty} \operatorname{Ev}^{\varepsilon}\left(\partial_{i+1}^{\varepsilon} P\right) t^{i}\right) t
$$

as it follows from (2.10.1) with $S:=1$. In particular, there is a decomposition $P=Q+R t$ where $Q \in \Lambda_{A / k}^{\varepsilon}$. Moreover, the decomposition is unique. Indeed, since $\Delta_{t}^{\varepsilon} 1=0$, the polynomial $R t$ belongs to the kernel of $\mathrm{Ev}^{\varepsilon}$. Hence, by (2.12.3), it follows from $P=Q+R t$, where $Q \in \Lambda_{A / k}^{\varepsilon}$, that $Q=\operatorname{Ev}^{\varepsilon}(P)$. So the decomposition (2.12.1) holds, and $\mathrm{Ev}^{\varepsilon}$ is the projection on the first factor. Obviously, therefore $\mathrm{Ev}^{\varepsilon}$ is the identity of $\Lambda_{A / k}^{\varepsilon}$ and $A\langle t\rangle t$ is the kernel of $\mathrm{Ev}^{\varepsilon}$.

Finally, since $\Lambda^{\varepsilon}=\Gamma^{\varepsilon}$, the equation (2.12.2) for $Q \in \Lambda^{\varepsilon}$ and $R \in A\langle t\rangle$ follows from (2.10.4) with $P:=Q$ and $S:=1$.

2.13. Definition. Consider the graded $k$-subalgebra $\Lambda_{A / k}^{\varepsilon}$ of $A\langle t\rangle$ defined in Proposition (2.12). It is $\Delta_{t}^{\varepsilon}$-invariant, and so $\Delta_{t}^{\varepsilon}$ defines, by restriction, an $\varepsilon$-derivation in $\Lambda_{A / k}^{\varepsilon}$. The induced $\varepsilon$-derivation will be denoted $d_{t}^{\varepsilon}$.

Clearly, the component of degree 0 in $\Lambda_{A / k}^{\varepsilon}$ is equal to $A$. The component of degree 1 is the kernel of the map $\partial_{1}^{\varepsilon}$. The latter map is simply the multiplication map $A\langle t\rangle^{1} \rightarrow A$. So the component of degree 1 is the submodule $I_{A / k}$ considered in $(2.1)$.

The map $\mathrm{Ev}^{\varepsilon}$ induces, by (2.12.1), an isomorphism of graded $A$-modules with $k$-linear endomorphisms,

$$
\left(\Lambda_{A / k}, d_{t}\right) \stackrel{\sim}{\longrightarrow}\left(\Lambda_{A / k}^{\varepsilon}, d_{t}^{\varepsilon}\right)
$$

The inverse map is the composition $\Lambda_{A / k}^{\varepsilon} \rightarrow A\langle t\rangle \rightarrow \Lambda_{A / k}$. Thus any Leibniz differential is of the form $Q\left(d_{t}\right)(1)$ with a uniquely determined polynomial $Q \in$ $\Lambda_{A / k}^{\varepsilon}$. In degree 0 , the map is the identity of $A$. In degree 1, the map is the identification of $I_{A / k}$ and $\Lambda_{A / k}^{1}$.

Since $\Lambda_{A / k}^{\varepsilon}$ is a graded $k$-algebra over $A$, the isomorphism (2.13.1) induces a structure on $\Lambda_{A / k}$ as a graded $k$-algebra over $A$. The product of Leibniz differentials $\omega, \pi$ with respect to the induced structure will be denoted $\omega . \pi$. Note that the product depends on the given $\varepsilon \in k$. It will be called the $\varepsilon$-product in $\Lambda_{A / k}$.

Since $d_{t}^{\varepsilon}$ is an $\varepsilon$-derivation of $\Lambda_{A / k}^{\varepsilon}$, it follows that the endomorphism $d_{t}$ is an $\varepsilon$-derivation with respect to the $\varepsilon$-product in $\Lambda_{A / k}$. In particular, we have the equation $d_{t}(a \pi)=d_{t} a . \pi+a d_{t} \pi$. It follows from (2.4.2) that the $\varepsilon$-product $\rho . \pi$ for $\rho \in \Lambda_{A / k}^{1}$ is equal to the product considered in (2.4), and hence independent of $\varepsilon$. 
Again, since $d_{t}$ is an $\varepsilon$-derivation with respect to the $\varepsilon$-product, we obtain from (1.9), for $P \in A\langle t\rangle$ and $\omega, \pi \in \Lambda_{A / k}$, the equations

$$
\begin{aligned}
& P\left(d_{t}\right)(\omega \cdot \pi)=\sum_{i=0}^{\infty} \partial_{i}^{\varepsilon} P\left(d_{t}\right)\left(\varepsilon_{\mathrm{gr}}^{i} \omega\right) \cdot d_{t}^{i} \pi \\
& P\left(d_{t}\right)(\omega) \cdot \pi=\sum_{i=0}^{\infty}(-1)^{i} \varepsilon^{i(i-1) / 2} \partial_{i}^{\varepsilon} P\left(d_{t}\right)\left(\varepsilon_{\mathrm{gr}}^{i} \omega \cdot d_{t}^{i} \pi\right) .
\end{aligned}
$$

Note that (2.13.3) characterizes the $\varepsilon$-product. Indeed, any Leibniz differential is of the form $P\left(d_{t}\right)(1)$, and from (2.13.3) we obtain the equation

$$
P\left(d_{t}\right)(1) . \pi=\sum_{i=0}^{\infty}(-1)^{i} \varepsilon^{i(i-1) / 2} \partial_{i}^{\varepsilon} P\left(d_{t}\right)\left(d_{t}^{i} \pi\right) .
$$

Note that (2.13.4) for $Q \in \Lambda_{A / k}^{\varepsilon}$ specializes to the equation

$$
Q\left(d_{t}\right)(1) \cdot \pi=Q\left(d_{t}\right)(\pi) .
$$

With the $\varepsilon$-product, the algebra $\Lambda_{A / k}$ is a $k$-algebra over $A$, and in particular an $A$ - $A$-module. Left multiplication by elements of $A$ is the multiplication given by the structure on $\Lambda_{A / k}$ as a left $A$-module. In general, right multiplication by elements of $A$ depends on $\varepsilon$. For instance, since $d_{t}$ is an $\varepsilon$-derivation, we have that

$$
d_{t}^{2} a . b=d_{t}\left(d_{t} a . b\right)-\varepsilon d_{t} a . d_{t} b .
$$

The products $d_{t} a . b$ and $d_{t} a . d_{t} b$ are independent of $\varepsilon$, and it is easy to give conditions under which $d_{t} a . d_{t} b \neq 0$ (cf. (3.14)). The structure on $\Lambda_{A / k}$ as a right $A$-module via the $\varepsilon$-product is given by (2.13.4). For instance, with $P:=t^{2} a$ and $\pi:=b$ we have that $(t+h)^{2}=t^{2}+(1+\varepsilon) t h+h^{2}$, and it follows that

$$
d_{t}^{2} a . b=d_{t}^{2}(a b)-(1+\varepsilon) d_{t}\left(a d_{t} b\right)+\varepsilon a d_{t}^{2} b .
$$

The $A$-module $\Lambda_{A / k}^{2}$ is generated as a left $A$-module by all differentials of the form $d_{t}\left(a d_{t} b\right)$. With respect to the $\varepsilon$-product, we have that $d_{t}\left(a d_{t} b\right)=d_{t} a \cdot d_{t} b+a d_{t}^{2} b$. Hence $\Lambda_{A / k}^{2}$ is generated by the differentials of the following two forms:

$$
d_{t}^{2} a, \quad d_{t} a . d_{t} b .
$$

Similarly, $\Lambda_{A / k}^{3}$ is generated as a left $A$-module by the differentials of the four forms:

$$
d_{t}^{3} a, \quad d_{t}^{2} a . d_{t} b, \quad d_{t} a . d_{t}^{2} b, \quad d_{t} a \cdot d_{t} b \cdot d_{t} c .
$$

2.14. Remark. The algebra $\Lambda_{A / k}$ with the $\varepsilon$-product and the $\varepsilon$-derivation $d_{t}$ has the following universal property:

Given a graded $k$-algebra $\Gamma$ over $A$ and an $\varepsilon$-derivation $D$ of $\Gamma$. Then there is a unique homomorphism of $k$-algebras over $A$ with $k$-linear endomorphisms,

$$
\left(\Lambda_{A / k}, d_{t}\right) \rightarrow(\Gamma, D) .
$$

Indeed, by (2.8), there is a unique homomorphism (2.14.1) of $A$-modules with $k$ linear endomorphisms such that the unity 1 of $\Lambda_{A / k}$ is mapped to the unity 1 of $\Gamma$. It is the map induced by $P \mapsto P(D)(1)$. Hence, under the isomorphism (2.13.1), it is the map $E: \Lambda_{A / k}^{\varepsilon} \rightarrow \Gamma$ defined by $E(Q)=Q(D)(1)$. Thus it suffices to prove that $E$ is a homomorphism with respect to the multiplications in the algebras. Let 
$Q$ and $R$ be polynomials in $\Lambda_{A / k}^{\varepsilon}$. Since $\operatorname{Ev}^{\varepsilon}(R)=R$, we have that $R=R\left(d_{t}^{\varepsilon}\right)(1)$. Hence, by (2.12.2), we have that

$$
Q R=Q\left(d_{t}^{\varepsilon}\right)(R)=Q\left(d_{t}^{\varepsilon}\right) R\left(d_{t}^{\varepsilon}\right)(1) .
$$

Therefore, since $E$ is a homomorphism of $A$-modules with endomorphisms, it follows that

$$
E(Q R)=Q(D) R(D)(1)=Q(D)(E(R)) .
$$

By (2.12), we have that $\partial_{i}^{\varepsilon} Q=0$ for $i>0$. Hence, from (2.9.1), applied with $Q:=P$ and $x:=E(R)$, it follows that

$$
Q(D)(E(R))=Q(D)(1) E(R)=E(Q) E(R) .
$$

Hence $E(Q R)=E(Q) E(R)$, and the assertion has been proved.

2.15. Note. By Proposition (2.12), the polynomials $Q$ of the subalgebra $\Lambda_{A / k}^{\epsilon}$ are characterized by the equations $\partial_{i}^{\varepsilon} Q=0$ for $i>0$. If $\varepsilon=0$, then, by (1.12), we have that $\partial_{i}^{\varepsilon}=\left(\partial_{1}^{\varepsilon}\right)^{i}$. Hence, for $\varepsilon=0$, the subalgebra $\Lambda_{A / k}^{\epsilon}$ is the kernel of the map $\partial_{1}^{\varepsilon}$. In fact, for $\varepsilon=0$, it is easily seen that the subalgebra $\Lambda_{A / k}^{\varepsilon}$ of $A\langle t\rangle$ is equal to $A \oplus A\langle t\rangle I_{A / k}$. Hence, for $\varepsilon=0$, right multiplication by elements of $A$ is given by the canonical structure of $\Lambda_{A / k}$ as a right $A$-module defined in (2.2).

If $\varepsilon=1$ and $k$ contains the field of rational numbers, then, again by (1.12), we have that $\Lambda_{A / k}^{\epsilon}$ is the kernel of $\partial_{1}^{\varepsilon}$.

\section{KÄHLER DIFFERENTIALS}

The $A$-module of Kähler differentials is the maximal quotient of the module of Leibniz differentials having the property that the endomorphism $d_{t}$ induces a differential operator of order at most 1 on the quotient. This property the Kähler differentials share with the higher order differentials obtained from the most interesting examples. More precisely, the module of Kähler differentials is the quotient $\Omega_{A / k}=\Lambda_{A / k} / \mathfrak{A}$, where $\mathfrak{A}$ is the smallest $d_{t}$-invariant $A$-submodule of $\Lambda_{A / k}$ containing the elements

$$
d_{t}(b a \pi)-b d_{t}(a \pi)-a d_{t}(b \pi)+a b d_{t}(\pi),
$$

for all $a, b$ in $A$ and $\pi$ in $\Lambda_{A / k}$. The Kähler differentials form a graded $A$-module and the $k$-linear endomorphism $d_{t}$ induces a $k$-linear endomorphism $d$ on $\Omega_{A / k}$. Denote the $n$ 'th graded piece of $\Omega_{A / k}$ by $\Omega_{A / k}^{n}$. We prove that, for every $n \geq 1$, we have an exact sequence

$$
0 \rightarrow \Omega_{A / k}^{1} \otimes_{A} \Omega_{A / k}^{n} \stackrel{i}{\rightarrow} \Omega_{A / k}^{n+1} \stackrel{r}{\rightarrow} \Omega_{A / k}^{n} \rightarrow 0
$$

of $A$ - $A$-linear maps that is split by right $A$-linear maps.

For every element $\varepsilon$ of $k$ we show that $\mathfrak{A}$ is a two sided ideal in $\Lambda_{A / k}$ with respect to the multiplication induced by $\Lambda_{A / k}^{\varepsilon}$. Hence $\Omega_{A / k}$ inherits the multiplicative structure of $\Lambda_{A / k}^{\varepsilon}$ and $d$ becomes an $\varepsilon$-derivation with respect to this structure. We denote by $\Omega_{A / k}^{\varepsilon}$ the Kähler differentials with the structure inherited from $\Lambda_{A / k}^{\varepsilon}$ and show that $\left(\Omega_{A / k}^{\varepsilon}, d\right)$ is universal for graded $k$-algebras over $A$ with an $\varepsilon$-derivation $D$ satisfying $D(b 1) a=a D(b 1)$, for all $a$ and $b$ in $A$.

When the ring $A$ is commutative, the first graded piece of the Kähler differentials is the $A$-module $\Omega_{A / k}^{1}$ of first order Kähler differentials of the $k$-algebra $A$. We show that, when the first order Kähler differentials form a finitely generated free 
$A$-module, the graded pieces of the Kähler differentials are free $A$-modules, and we describe explicitly bases for these modules.

We also define the Kähler differentials of an $A$-module and show how a connection on the module gives rise to a map on the Kähler differentials of the module with properties similar to a derivation.

3.1. Lemma. Let $\mathcal{R}$ be a subset of $\Lambda_{A / k}^{1}$. Denote by $\mathfrak{A}(\mathcal{R})$ the subset of $\Lambda_{A / k}$ consisting of all sums of Leibniz differentials of the following form:

$$
P\left(d_{t}\right)(\rho . \pi) \quad \text { for } P \in A\langle t\rangle, \rho \in \mathcal{R}, \pi \in \Lambda_{A / k} .
$$

Then $\mathfrak{A}(\mathcal{R})$ is a homogeneous $d_{t}$-invariant $A$-A-submodule of $\Lambda_{A / k}$. The component of degree 0 of $\mathfrak{A}(\mathcal{R})$ vanishes and the component of degree 1 is the A-A-submodule of $\Lambda_{A / k}^{1}$ generated by $\mathcal{R}$. Finally, for any $\varepsilon$, we have that $\mathfrak{A}(\mathcal{R})$ is an ideal with respect to the $\varepsilon$-product in $\Lambda_{A / k}$.

Proof. We have that $\mathfrak{A}(\mathcal{R})$ is a right $A$-submodule because the map $d_{t}$ is right $A$ linear in positive degree and the product $\rho . \pi$ is right $A$-linear. The remaining parts of the first assertion are obvious.

Since the elements of $\mathcal{R}$ are of degree 1 , the component of degree 0 in $\mathfrak{A}(\mathcal{R})$ contains only 0 . Similarly, all elements of degree 1 of $\mathfrak{A}(\mathcal{R})$ are sums of the form (3.1.1) with $P$ and $\pi$ of degree 0 . Hence the component of degree 1 is the $A-A$ submodule generated by $\mathcal{R}$.

To prove the final assertion, we have to prove, for any element (3.1.1) and any $\omega \in \Lambda_{A / k}$, that the following two $\varepsilon$-products belong to $\mathfrak{A}(\mathcal{R})$ :

$$
\omega . P\left(d_{t}\right)(\rho . \pi), \quad P\left(d_{t}\right)(\rho . \pi) \cdot \omega .
$$

For the first product, write $\omega=Q\left(d_{t}\right)(1)$ with a polynomial $Q \in \Lambda_{A / k}^{\varepsilon}$. Then it follows from (2.13.5), that

$$
\omega . P\left(d_{t}\right)(\rho . \pi)=Q\left(d_{t}\right) P\left(d_{t}\right)(\rho . \pi) .
$$

Hence the first product is of the form (3.1.1).

Consider the second product. Since $\rho$ is of degree 1, we have by (2.13.3) that

$$
P\left(d_{t}\right)(\rho \cdot \pi) \cdot \omega=\sum_{i=0}^{\infty}(-1)^{i} \varepsilon^{i(i-1) / 2} \varepsilon^{i} \partial_{i}^{\varepsilon} P\left(d_{t}\right)\left(\rho \cdot \varepsilon_{\mathrm{gr}}^{i} \pi \cdot d_{t}^{i} \omega\right) .
$$

Hence the second product is a sum of terms of the form (3.1.1). Thus both products belong to $\mathfrak{A}(\mathcal{R})$, and the proof of the lemma is complete.

3.2. Definition. Let $\mathcal{R}$ be the subset of $\Lambda_{A / k}^{1}$ formed by all elements of the following form:

$$
\rho=\left(d_{t} b\right) a-a\left(d_{t} b\right) \quad \text { for } a, b \in A .
$$

Let $\mathfrak{A}:=\mathfrak{A}(\mathcal{R})$ be the $A$-A-submodule of $\Lambda_{A / k}$ defined (3.1). Denote by $\Omega_{A / k}$ the quotient of $\Lambda_{A / k}$ modulo $\mathfrak{A}$. Since $\mathfrak{A}$ is a homogeneous $A$ - $A$-submodule, the quotient $\Omega_{A / k}$ is a graded $A$-A-module. Since $\mathfrak{A}(\mathcal{R})$ is $d_{t}$-invariant, the endomorphism $d_{t}$ induces a $k$-linear endomorphism of degree 1 of the quotient

$$
d: \Omega_{A / k} \rightarrow \Omega_{A / k} \text {. }
$$

In fact, the endomorphism $d$ is right $A$-linear in positive degrees since $d_{t}$ is right $A$-linear in positive degrees. 
The elements of $\Omega_{A / k}$ will be called (free) Kähler differentials. When it is necessary to indicate the dependence on the given $k$-algebra $A$, we will write $d_{A / k}$ for $d$.

Since $\mathfrak{A}^{0}=0$, the quotient map defines an isomorphism of $A=\Lambda_{A / k}^{0}$ onto the Kähler differentials of degree 0 , and we will always identify $\Omega_{A / k}^{0}$ and $A$. In particular, the unit 1 of $A$ will be considered as an element of $\Omega_{A / k}$. The endomorphism $d$ of $\Omega_{A / k}$ is induced by the endomorphism $d_{t}$ of $\Lambda_{A / k}$, and $d_{t}$ is induced by left multiplication by $t$ in $A\langle t\rangle$. Hence, under the composition $A\langle t\rangle \rightarrow \Lambda_{A / k} \rightarrow \Omega_{A / k}$, the polynomial $P$ is mapped to $P(d)(1)$. In particular, every Kähler differential is of the form $P(d)(1)$ for a suitable polynomial $P$.

Consider the component $\Omega_{A / k}^{1}$ of degree 1 . It is the quotient of $\Lambda_{A / k}^{1}$ modulo $\mathfrak{A}^{1}$. Since $d_{t}: A \rightarrow \Lambda_{A / k}^{1}$ is a module derivation, it follows that $d: A \rightarrow \Omega_{A / k}^{1}$ is a module derivation, that is,

$$
d(a b)=(d a) b+a(d b) .
$$

Note that, by the choice of $\mathcal{R}$, we have in $\Omega_{A / k}^{1}$ the equation, for $a, b \in A$,

$$
(d b) a=a(d b) .
$$

In particular, when $A$ is commutative, it follows that the left structure and the right structure of $\Omega_{A / k}^{1}$ as an $A$-module coincide.

3.3. Definition. As observed in (2.1), the $A-A$-module $\Lambda_{A / k}^{1}$ can be identified with the kernel $I_{A / k}$ of the multiplication map $A \otimes_{k} A \rightarrow A$. Under the identification we have that $d_{t} b=1 \otimes_{k} b-b \otimes_{k} 1$, and

$$
\left(d_{t} b\right) a-a\left(d_{t} b\right)=1 \otimes_{k} b a-b \otimes_{k} a-a \otimes_{k} b+a b \otimes_{k} 1 .
$$

Hence $\mathfrak{A}^{1} \subseteq I_{A / k}$ can be identified with the $A$ - $A$-submodule generated by all elements of the form (3.3.1). Let $P_{A / k}^{1}$ be the $A$-A-module defined as the quotient

$$
P_{A / k}^{1}:=\left(A \otimes_{k} A\right) / \mathfrak{A}^{1} .
$$

Then there is an exact sequence of $A$-A-linear maps

$$
0 \rightarrow \Omega_{A / k}^{1} \stackrel{i^{1}}{\longrightarrow} P_{A / k}^{1} \stackrel{r^{1}}{\longrightarrow} A \rightarrow 0
$$

where $i^{1}$ is the inclusion and $r^{1}$ is the surjection induced by the multiplication map $A \otimes_{k} A \rightarrow A$. Moreover, $r^{1}$ is split by the left $A$-linear map represented by $a \mapsto a \otimes_{k} 1$ and by the right $A$-linear map represented by $a \mapsto 1 \otimes_{k} a$.

For any $A$-module $M$, the tensor product $P_{A / k}^{1} \otimes_{A} M$ is an $A$-module and a quotient of $A \otimes_{k} A \otimes_{A} M=A \otimes_{k} M$. Define $P_{A / k}^{1}(M):=P_{A / k}^{1} \otimes_{A} M$, and let $d_{M}^{1}: M \rightarrow P_{A / k}^{1}(M)$ be the $k$-linear map represented by $x \mapsto 1 \otimes_{k} x$. Then, since the map $P_{A / k}^{1} \rightarrow A$ has a right $A$-linear section, we obtain from (3.3.3) an exact sequence of $A$-modules

$$
0 \rightarrow \Omega_{A / k}^{1} \otimes_{A} M \stackrel{i_{M}^{1}}{\longrightarrow} P_{A / k}^{1}(M) \stackrel{r_{M}^{1}}{\longrightarrow} M \rightarrow 0 .
$$

Clearly, the composition $r_{M}^{1} d_{M}^{1}$ is the identity map of $M$. Moreover, for $a \in A$ and $x \in M$, we have that $1 \otimes_{k} 1 \otimes_{A} a x-a \otimes_{k} 1 \otimes_{A} x=d_{t} a \otimes_{A} x$. It follows that

$$
d_{M}^{1}(a x)-a d_{M}^{1}(x)=i_{M}^{1}\left(d a \otimes_{A} x\right) .
$$

The $A$-module $P_{A / k}^{1}(M)$ is called the module of first order principal parts. 
View the tensor product $A \otimes_{k} A$ as the $k$-algebra $A^{\mathrm{e}}:=A \otimes_{k} A^{\mathrm{op}}$. The multiplication in the algebra $A^{\mathrm{e}}$ will be denoted by a dot. It is given by the equation $(a \otimes b) \cdot(x \otimes y):=a x \otimes y b$. Thus $A$-A-submodules of $A \otimes_{k} A$ correspond to left ideals of the algebra $A^{\mathrm{e}}$. In particular, $I_{A / k}$ is the left ideal generated by the elements (3.3.1). Right multiplication by $a$ in the $A$-A-module $A \otimes_{k} A$ corresponds to left multiplication by $1 \otimes_{k} a$ in the algebra $A^{\mathrm{e}}$. Hence, for the element (3.3.1) we have the equation

$$
\left(d_{t} b\right) a-a\left(d_{t} b\right)=\left(1 \otimes_{k} a\right) \cdot d_{t} b-\left(a \otimes_{k} 1\right) \cdot d_{t} b=d_{t} a \cdot d_{t} b .
$$

It follows that $\mathfrak{A}^{1}$ is the left ideal of $A^{\mathrm{e}}$ generated by all products $d_{t} a \cdot d_{t} b$.

If $A$ is commutative, then $A^{\mathrm{e}}$ is the commutative algebra $A \otimes_{k} A$ and $I_{A / k}$ is an ideal. Moreover, $\mathfrak{A}^{1}$ is the square $I_{A / k}^{2}$. Hence, when $A$ is commutative, the $A$-module $\Omega_{A / k}^{1}$ is the usual module $I_{A / k} / I_{A / k}^{2}$ of Kähler differentials of degree 1 , and $P_{A / k}^{1}(M)$ is the usual module of first order principal parts.

3.4. Lemma. Let $\varphi: M \rightarrow M^{\prime}$ be a k-linear map of A-modules. Then the following four conditions are equivalent:

(i) For all $b \in A$, the map $\varphi b_{M}-b_{M^{\prime}} \varphi$ is an A-linear map $M \rightarrow M^{\prime}$.

(ii) For all $a, b \in A$ and $x \in M$, we have the equation

$$
\varphi(b a x)-b \varphi(a x)=a \varphi(b x)-a b \varphi(x) .
$$

(iii) There exists an A-linear map $u: P_{A / k}^{1}(M) \rightarrow M^{\prime}$ such that $\varphi=u d_{M}^{1}$.

(iv) There exists an A-linear map, $\Omega_{A / k}^{1} \otimes_{A} M \rightarrow M^{\prime}$, denoted $\rho \otimes_{A} x \mapsto \rho . x$, such that, for all $a \in A$ and $x \in M$, we have that

$$
\varphi(a x)=d a . x+a \varphi(x) .
$$

Proof. Clearly, (i) and (ii) are equivalent.

Assume (ii). Consider the $A$-linear extension $A \otimes_{k} M \rightarrow M^{\prime}$ defined by $a \otimes_{k} x \mapsto$ $a \varphi(x)$. It follows from the condition in (ii) that the extension vanishes on the submodule defining the quotient $P_{A / k}^{1}(M)$. Hence the extension induces an $A$ linear map $u: P_{A / k}^{1}(M) \rightarrow M^{\prime}$. Clearly, $\varphi=u d_{M}^{1}$. Hence (iii) holds.

Assume (iii). Define $\rho . x:=u i_{M}\left(\rho \otimes_{A} x\right)$. Then it follows from (3.3.5) that (iv) holds.

Assume (iv). Then the left side of (3.4.1) is equal to $d b . a x$ and the right side is equal to $a(d b . x)$. Therefore, since $(d b) a=a(d b)$, it follows that (ii) holds.

Hence the equivalence of the four conditions have been shown.

3.5. Definition. A $k$-linear map $\varphi: M \rightarrow M^{\prime}$ of $A$-modules satisfying the equivalent conditions of Lemma (3.4) is said to be a differential operator of order at most 1 (for the $k$-algebra $A$ ), cf. [9, 16.8, p. 39]. It follows from the lemma, for an $A$-module $M$, that the map $d_{M}^{1}: M \rightarrow P_{A / k}^{1}(M)$ is the universal differential operator of order at most 1 defined on $M$.

3.6. Definition. The $k$-linear map $d: \Omega_{A / k} \rightarrow \Omega_{A / k}$ is a differential operator of order at most 1 , that is, for $a, b \in A$ and $\omega \in \Omega_{A / k}$, we have the equation

$$
d(b a \omega)-b d(a \omega)-a d(b \omega)+a b d(\omega)=0 .
$$


Indeed, $\omega$ is the image of a Leibniz differential $\pi$. So the expression on the left side of (3.6.1) is the image of the following expression in $\Lambda_{A / k}$ :

$$
d_{t}(b a \pi)-b d_{t}(a \pi)-a d_{t}(b \pi)+a b d_{t}(\pi) .
$$

By (2.4.2), the latter expression is equal to

$$
d_{t} b . a \pi-a d_{t} b . \pi=\left(d_{t}(b) a-a d_{t}(b)\right) \cdot \pi .
$$

It follows that the expression belongs to $\mathfrak{A}$. Thus (3.6.1) holds.

Therefore, by Lemma (3.4), there is an induced $A$-linear product,

$$
i: \Omega_{A / k}^{1} \otimes_{A} \Omega_{A / k} \rightarrow \Omega_{A / k}
$$

denoted $\rho \otimes_{A} \omega \mapsto \rho . \omega$. As the tensor product is over $A$, we have that $(\rho a) \cdot \omega=$ $\rho$. (aw). Clearly, the map $i$ is homogeneous of degree 1 . In degree 0 , it is the identity of $\Omega_{A / k}^{1}$. Moreover, since the map $d: \Omega_{A / k} \rightarrow \Omega_{A / k}$ is right $A$-linear, it follows easily that $i$ is right $A$-linear in all degrees. By (3.4.2) we have that

$$
d a . \omega=d(a \omega)-a d \omega .
$$

3.7. Lemma. Let $w: N \rightarrow N^{\prime}$ be a k-linear map of right $A$-modules. Then, in the notation of Lemma (2.5), the right A-linear map $w_{t}: N \otimes_{A} \Lambda_{A / k} \rightarrow N^{\prime} \otimes_{A} \Lambda_{A / k}$ induces a right A-linear map of the quotients

$$
\bar{w}: N \otimes_{A} \Omega_{A / k} \rightarrow N^{\prime} \otimes_{A} \Omega_{A / k},
$$

if and only if, for all $x \in N$ and $a, b \in A$, we have the equation in $N^{\prime}$

$$
w(x) b a-w(x b) a-w(x a) b+w(x a b)=0 .
$$

Moreover, if (3.7.2) holds, then the induced map $\bar{w}$ is homogeneous of degree -1 , and equations parallel to (2.5.2) and (2.5.3) hold for $\bar{w}$.

Proof. For $n \geq 0$, let $w_{n}: N \otimes_{A} \Lambda_{A / k}^{n+1} \rightarrow N^{\prime} \otimes_{A} \Lambda_{A / k}^{n}$ be the component of degree $n$ of the map $w_{t}$ of $(2.5)$. Let $\rho:=\left(d_{t} b\right) a-a\left(d_{t} b\right)$ with $a, b \in A$. Then the left side of (3.7.2) is the value $w_{0}\left(x \otimes_{A} \rho\right)$. Since $w_{0}$ is right $A$-linear, it follows that $w_{0}$ induces a right $A$-linear map $\bar{w}_{0}: N \otimes_{A} \Omega_{A / k}^{1} \rightarrow N^{\prime}$ if and only if (3.7.2) holds.

Assume that equation (3.7.2) holds, and consider $w_{n}$ for $n \geq 1$. Denote by $N^{\prime} \otimes \mathfrak{A}$ the image of $N^{\prime} \otimes_{A} \mathfrak{A}$ in $N^{\prime} \otimes_{A} \Lambda_{A / k}$. To prove that $w_{n}$ induces a map on the quotients, we have to prove, for an element $\omega=P\left(d_{t}\right)(\rho . \pi)$ in $\mathfrak{A}^{n+1}$, that the value $w_{n}\left(x \otimes_{A} \omega\right)$ belongs to the submodule $N^{\prime} \otimes \mathfrak{A}^{n}$. Clearly, we may assume that $P$ is homogeneous. Assume first that $P$ is of positive degree. Then, since the tensor product is over $A$, we may assume that $P=t Q$. Hence, by (2.5.2),

$$
w_{n}\left(x \otimes_{A} \omega\right)=w_{n}\left(x \otimes_{A} d_{t} Q\left(d_{t}\right)(\rho . \pi)\right)=w(x) \otimes_{A} Q\left(d_{t}\right)(\rho . \pi),
$$

and so $w_{n}\left(x \otimes_{A} \omega\right)$ belongs to $N^{\prime} \otimes \mathfrak{A}$. Assume next that $P$ is of degree 0 , that is, $P$ is an element $c$ of $A$. Then $\omega=c \rho$. $\pi$. It follows from (2.5.3) that

$$
w_{n}\left(x \otimes_{A} c \rho . \pi\right)=w_{0}\left(x c \otimes_{A} \rho\right) \otimes_{A} \pi .
$$

As noted in the beginning of the proof, it follows from (3.7.2) that $w_{0}(x c \otimes \rho)=0$. Hence $w_{n}\left(x \otimes_{A} \omega\right)=0$. In particular, $w_{n}\left(x \otimes_{A} \omega\right)$ belongs to $N^{\prime} \otimes \mathfrak{A}$.

Thus the lemma has been proved. 
3.8. Definition. Obviously, the condition (3.7.2) holds when $w$ is the identity map of $A$. Hence it follows from Lemma (3.7) that the map $r_{t}$ of (2.6) induces a right $A$-linear map of degree -1 on the quotients. It will be denoted

$$
r: \Omega_{A / k} \rightarrow \Omega_{A / k} \text {. }
$$

The induced map $r$ is $A$-A-linear, because $r$ is $A$ - $A$-linear.

Take as $k$-linear map $w$ in (3.7) the map $-d: A \rightarrow \Omega_{A / k}^{1}$ to obtain a right $A$-linear map $w_{t}: \Lambda_{A / k} \rightarrow \Omega_{A / k}^{1} \otimes_{A} \Lambda_{A / k}$ of degree -1 . The condition (3.7.2) holds, because $d: A \rightarrow \Omega_{A / k}^{1}$ is a module derivation and $(d b) a=a(d b)$. Hence it follows from Lemma (3.7) that the right $A$-linear map $w_{t}$ induces a right $A$-linear map of degree -1 on the quotients. It will be denoted

$$
s: \Omega_{A / k} \rightarrow \Omega_{A / k}^{1} \otimes_{A} \Omega_{A / k} .
$$

Clearly, the maps $r$ and $s$ and the map $i$ of (3.6) are induced on the quotients by the maps $r_{t}, s_{t}$ and $i_{t}$ of (2.6) and (2.4). As a consequence, when $n \geq 1$, we obtain from (2.6) the following equations, for $a \in A, \rho \in \Omega_{A / k}^{1}$ and $\omega \in \Omega_{A / k}^{n}$ :

$$
\begin{gathered}
r(a d \omega)=a \omega, \quad r(\rho . \omega)=0 . \\
s(a d \omega)=-d a \otimes_{A} \omega, \quad s(\rho . \omega)=\rho \otimes_{A} \omega .
\end{gathered}
$$

3.9. Proposition. Assume that $n \geq 1$. Then the following sequence of A-A-linear maps is exact:

$$
0 \longrightarrow \Omega_{A / k}^{1} \otimes_{A} \Omega_{A / k}^{n} \stackrel{i}{\longrightarrow} \Omega_{A / k}^{n+1} \stackrel{r}{\longrightarrow} \Omega_{A / k}^{n} \longrightarrow 0,
$$

and split by the right $A$-linear maps $d$ and $s$.

Proof. The proof is identical to the proof of Proposition (2.7)

3.10. Definition. By Lemma (3.1), the homogeneous $A$ - $A$-submodule $\mathfrak{A}$ of $\Lambda_{A / k}$ defining $\Omega_{A / k}$ is, for any $\varepsilon$, an ideal with respect to the $\varepsilon$-product. Therefore, when $\Lambda_{A / k}$ is considered as a graded $k$-algebra over $A$ via the $\varepsilon$-product, there is a unique product in $\Omega_{A / k}$ such that the canonical map

$$
\Lambda_{A / k} \rightarrow \Omega_{A / k}
$$

is a homomorphism of $k$-algebras over $A$. The product in $\Omega_{A / k}$ is called the $\varepsilon$ product. The endomorphism $d$ of $\Omega_{A / k}$ is an $\varepsilon$-derivation with respect to the $\varepsilon$ product. When $\Omega_{A / k}$ is considered as a graded $k$-algebra over $A$ via the $\varepsilon$-product, it will be denoted $\Omega_{A / k}^{\varepsilon}$.

3.11. Remark. Let $M$ be an $A$-module and let $\varphi$ be a $k$-linear endomorphism of $M$. Let $u$ be an element in the kernel of $\varphi$. Consider the map of $A$-modules with $k$-linear endomorphisms of $(2.8)$

$$
E:\left(\Lambda_{A / k}, d_{t}\right) \rightarrow(M, \varphi)
$$

Then the map $E$ induces a map from the quotient

$$
\left(\Omega_{A / k}, d\right) \rightarrow(M, \varphi),
$$

if and only if the restriction of $\varphi$ to $\Lambda(M, \varphi, u)$ is a differential operator of order at most 1, that is, for all $a, b \in A$ and all $x \in \Lambda(M, \varphi, u)$, the following equation holds:

$$
\varphi(b a x)-b \varphi(a x)-a \varphi(b x)+a b \varphi(x)=0 .
$$


Indeed, since $E$ is a map of $A$-modules with endomorphisms, it vanishes on the $A$-submodule $\mathfrak{A}$ defining $\Omega_{A / k}$ if and only if it vanishes on all Leibniz differentials of the form $\rho$. $\pi$ where $\rho=\left(d_{t} b\right) a-a\left(d_{t} b\right)$. By definition of the product $\rho$. $\pi$, we have that

$$
\rho . \pi=d_{t}(b a \pi)-b d_{t}(a \pi)-a d_{t}(b \pi)+a b d_{t} \pi .
$$

Hence, with $x=E(\pi)$, the left hand side of (3.11.3) is the value $E(\rho . \pi)$. Since the map $E: \Lambda_{A / k} \rightarrow \Lambda(M, \varphi, u)$ is surjective, it follows that $E$ vanishes on $\mathfrak{A}$, if and only if (3.11.3) holds.

With respect to the $\varepsilon$-product, the algebra $\Omega_{A / k}^{\varepsilon}$ has the following universal property:

Given a graded $k$-algebra $\Gamma$ over $A$ and an $\varepsilon$-derivation $D$ of $\Gamma$. Assume for all $a, b \in A$ that $D(b 1) a=a D(b 1)$. Then there is a unique homomorphism of $k$-algebras over $A$ with $k$-linear endomorphisms,

$$
\left(\Omega_{A / k}^{\varepsilon}, d\right) \rightarrow(\Gamma, D) .
$$

Indeed, by (2.14), there is a unique homomorphism of $k$-algebras over $A$ with $k$ linear endomorphisms

$$
\left(\Lambda_{A / k}, d_{t}\right) \rightarrow(\Gamma, D) .
$$

Since $D$ is an $\varepsilon$-derivation, we have, for all $a, b \in A$ and $x \in \Gamma$, that

$$
D(b a x)-b D(a x)-a D(b x)+a b D(x)=D(b 1) a x-a D(b 1) x=0 .
$$

In particular, the equation (3.11.3) with $\varphi:=D$ holds. Hence (3.11.5) descends to the map (3.11.4), and since (3.11.5) is a map of $k$-algebras over $A$, so is (3.11.4).

3.12. Proposition. Let $\left\{x_{1}, \ldots, x_{m}\right\}$ be a subset of $A$ such that the differentials $d x_{j}$ form a left $A$-basis for $\Omega_{A / k}^{1}$. Then $\Omega_{A / k}^{n}$ is a free left $A$-module with a basis of $m(m+1)^{n-1}$ elements. More precisely, let $\mathcal{X}_{1}$ be the subset $\left\{d x_{1}, \ldots, d x_{m}\right\}$ of $\Omega_{A / k}^{1}$ and, inductively, let $\mathcal{X}_{n+1}$ be the subset of $\Omega_{A / k}^{n+1}$ consisting of elements of any of the following two forms:

$$
d \xi \quad \text { or } \quad d x_{j} . \xi \quad \text { for } \xi \in \mathcal{X}_{n}, j=1, \ldots, m .
$$

Then the set $\mathcal{X}_{n}$ is a left $A$-basis for $\Omega_{A / k}^{n}$.

Proof. By hypothesis, the set $\mathcal{X}_{1}$ is a left $A$-basis for $\Omega_{A / k}^{1}$. Since $a(d x)=(d x) a$, the set $\mathcal{X}_{1}$ is also a right $A$-basis for $\Omega_{A / k}^{1}$. Hence, by induction on $n$, the assertion follows from the exact sequence of Proposition (3.9).

3.13. Corollary. Assume that $A$ is commutative. Let $\left\{x_{1}, \ldots, x_{m}\right\}$ be a subset of $A$ such that the differentials $d x_{j}$ form an $A$-basis of $\Omega_{A / k}^{1}$. Then the set of all $\varepsilon$-products,

$$
\pi=d^{n_{1}} x_{j_{1}} \ldots d^{n_{l}} x_{j_{l}},
$$

where each $n_{i}>0$ and $n_{1}+\cdots+n_{l}=n$, form a left $A$-basis of $\Omega_{A / k}^{n}$.

Proof. Let $\Omega^{\prime}$ be the $A$-submodule of $\Omega_{A / k}$ generated by all products (3.13.1). Since $d$ is an $\varepsilon$-derivation of $\Omega_{A / k}^{\varepsilon}$, it follows for a product $\pi$ of the form (3.13.1) that $d \pi$ is a $k$-linear combination of similar products. Moreover, for $f \in A$ we have that $d f$ is an $A$-linear combination of the $d x_{j}$. Hence $d(f \pi)=d f . \pi+f d \pi$ belongs to $\Omega^{\prime}$. Thus the $A$-submodule $\Omega^{\prime}$ is $d$-invariant. In addition, it contains 1 , which is 
the empty product (3.13.1). Hence $\Omega^{\prime}$ contains all Kähler differentials $P(d)(1)$ for $P \in A\langle t\rangle$. Thus $\Omega^{\prime}=\Omega_{A / k}$.

The number of products (3.13.1) of degree $n$ is $m(m+1)^{n-1}$ when $n \geq 1$. Indeed, in the product (3.13.1) write $d^{n_{i}} x_{j_{i}}=d \cdots d\left(d x_{j_{i}}\right)$ to see that the products (3.13.1) of degree $n$ correspond bijectively to "formal" strings $\delta_{1} \cdots \delta_{n}$ where each symbol $\delta_{i}$ is either equal to $d$ or equal to a differential $d x_{j}$ and the last symbol $\delta_{n}$ is not equal to $d$. Clearly, the number of such formal strings is equal to $(m+1)^{n-1} m$. Therefore, since the number of products it equal to the rank of the free $A$-module $\Omega_{A / k}^{n}$, it follows that the products form an $A$-basis.

3.14. Note. Assume the setup of the corollary. The endomorphism $d$ of $\Omega_{A / k}$ is determined in the basis since $d$ is an $\varepsilon$-derivation and $d f$, for $f \in A$, has an expansion as an $A$-linear combination of the differentials $d x_{j}$. The coefficients in the expansion may be called the partial derivatives of $f$, denoted $f_{x_{j}}^{\prime}$. Note also that the $\varepsilon$-multiplication is determined in terms of the basis, since $d x_{j} . f=f d x_{j}$ and inductively, for $n \geq 1$,

$$
d^{n+1} x_{j} . f=d\left(d^{n} x_{j} . f\right)-\varepsilon^{n} d^{n} x_{j} . d f .
$$

By induction on $n$, it follows that $d^{n} x_{j} . f$ in the $A$-basis of products (3.13.1) has an expansion where the coefficients are $k$-linear combinations of iterations of partial derivatives of $f$.

For instance, for $n=2$ we obtain the equation

$$
\begin{aligned}
d^{2} x_{j} . f=d( & \left(x_{j} . f\right)-\varepsilon d x_{j} . d f \\
& =d\left(f d x_{j}\right)-\varepsilon d x_{j} . d f=f d^{2} x_{j}+\sum_{i=1}^{m} f_{x_{i}}^{\prime} d x_{i} . d x_{j}-\varepsilon \sum_{i=1}^{m} f_{x_{i}}^{\prime} d x_{j} . d x_{i} .
\end{aligned}
$$

In particular, for $f:=x_{i}$ it follows that

$$
d^{2} x_{j} \cdot x_{i}=x_{i} d^{2} x_{j}+d x_{i} . d x_{j}-\varepsilon d x_{j} . d x_{i} .
$$

Hence, if $m \geq 2$, the structure on $\Omega_{A / k}^{2}$ as a right $A$-module given by the $\varepsilon$-product is different from the structure as a left module.

3.15. Note. Let $M$ be an $A$-module. Then, since $\Omega_{A / k}$ is a graded $A$ - $A$-module, the tensor product

$$
\Omega_{A / k}(M):=\Omega_{A / k} \otimes_{A} M
$$

is a graded $A$-module. In addition, since the map $d$ in positive degrees and the maps $i, r$, and $s$ are right $A$-linear, there are induced maps for $n \geq 1$ :

$$
\begin{aligned}
& d_{M}: \Omega_{A / k}^{n}(M) \rightarrow \Omega_{A / k}^{n+1}(M), \quad s_{M}: \Omega_{A / k}^{n+1}(M) \rightarrow \Omega_{A / k}^{1} \otimes_{A} \Omega_{A / k}^{n}(M), \\
& r_{M}: \Omega_{A / k}^{n+1}(M) \rightarrow \Omega_{A / k}^{n}(M), \quad i_{M}: \Omega_{A / k}^{1} \otimes_{A} \Omega_{A / k}^{n}(M) \rightarrow \Omega_{A / k}^{n+1}(M),
\end{aligned}
$$

Moreover, from (3.9) we obtain, for $n \geq 1$, the exact sequence of $A$-linear maps

$$
0 \rightarrow \Omega_{A / k}^{1} \otimes_{A} \Omega_{A / k}^{n}(M) \stackrel{i_{M}}{\longrightarrow} \Omega_{A / k}^{n+1}(M) \stackrel{r_{M}}{\longrightarrow} \Omega_{A / k}^{n}(M) \rightarrow 0,
$$

which is split by the $k$-linear maps $d_{M}$ and $s_{M}$.

3.16. Note. For a right $A$-module $N$, let $\Omega_{A / k}[N]$ be the graded $k$-module defined by

$$
\Omega_{A / k}[N]:=N \otimes_{A} \Omega_{A / k} .
$$


When the exact sequence of (3.9) for $n \geq 1$ is tensored from the left with $N$, we obtain the exact sequence of $k$-linear maps,

$$
\Omega_{A / k}^{1}[N] \otimes_{A} \Omega_{A / k}^{n} \stackrel{i_{N}}{\longrightarrow} \Omega_{A / k}^{n+1}[N] \stackrel{r_{N}}{\longrightarrow} \Omega_{A / k}^{n}[N] \rightarrow 0,
$$

where $i_{N}:=1_{N} \otimes_{A} i$ and $r_{N}=1_{N} \otimes_{A} r$. The map $i_{N}$ is called the product, and we write $\xi \cdot \omega:=i_{N}\left(\xi \otimes_{A} \omega\right)$.

A connection in $N$ is a $k$-linear map $\nabla: N \rightarrow N \otimes_{A} \Omega_{A / k}^{1}$ such that, for $x \in N$ and $a \in A$,

$$
\nabla(x a)=\nabla(x) a+x \otimes_{A} d a .
$$

A connection $\nabla$ on $N$ extends uniquely to a $k$-linear endomorphism, homogeneous of degree 1 ,

$$
\nabla: \Omega_{A / k}[N] \rightarrow \Omega_{A / k}[N],
$$

such that, for $x \in N$ and $\omega \in \Omega_{A / k}$,

$$
\nabla\left(x \otimes_{A} \omega\right)=\nabla(x) \cdot \omega+x \otimes_{A} d \omega .
$$

Indeed, to define the extended map $\nabla$, it suffices to note that, by the property of a connection, we have that

$$
\nabla(x a) \cdot \omega+x a \otimes_{A} d \omega=\nabla(x) . a \omega+x \otimes_{A} d(a \omega) .
$$

For a given connection $\nabla$ of $N$, there is a $k$-linear homomorphism, homogeneous of degree -1 ,

$$
s_{\nabla}: N \otimes_{A} \Omega_{A / k} \rightarrow N \otimes_{A} \Omega_{A / k}^{1} \otimes_{A} \Omega_{A / k},
$$

obtained from Lemma (3.7) by taking as $w$ the map $-\nabla: N \rightarrow N \otimes_{A} \Omega_{A / k}^{1}$. Indeed, we have that

$$
-\nabla(x) b a+\nabla(x b) a+\nabla(x a) b-\nabla(x a b)=\left(x \otimes_{A} d b\right) a-x a \otimes_{A} d b=0,
$$

and consequently the necessary equation (3.7.2) holds.

The two equations asserted at the end of (3.7) are the following, for $x \in N$, $\rho \in \Omega_{A / k}^{1}$ and $\omega \in \Omega_{A / k}^{n}$ :

$$
\begin{aligned}
s_{\nabla}\left(x \otimes_{A} d \omega\right) & =-\nabla(x) \otimes_{A} \omega \text { for } n \geq 1, \\
s_{\nabla}(x \otimes \rho . \omega) & =x \otimes_{A} \rho \otimes_{A} \omega .
\end{aligned}
$$

It follows, for $n \geq 1$, that the following sequence of $k$-linear maps is exact:

$$
0 \rightarrow \Omega_{A / k}^{1}[N] \otimes_{A} \Omega_{A / k}^{n} \stackrel{i_{N}}{\longrightarrow} \Omega_{A / k}^{n+1}[N] \stackrel{r_{N}}{\longrightarrow} \Omega_{A / k}^{n}[N] \rightarrow 0,
$$

and split by the $k$-linear maps $\nabla$ and $s_{\nabla}$.

Finally, for the given $\varepsilon$, we have that $\Omega_{A / k}$ is a graded $k$-algebra $\Omega_{A / k}^{\varepsilon}$ with respect to the $\varepsilon$-product. Hence $\Omega_{A / k}[N]=N \otimes_{A} \Omega_{A / k}^{\varepsilon}$ is a graded right module $\Omega_{A / k}^{\varepsilon}[N]$ over the graded $k$-algebra $\Omega_{A / k}^{\varepsilon}$. The following equation, for $\xi \in \Omega_{A / k}^{\varepsilon}[N]$ and $\omega \in \Omega_{A / k}^{\varepsilon}$, follows easily from the definitions:

$$
\nabla(\xi \omega)=\nabla(\xi) \omega+\varepsilon_{\mathrm{gr}}(\xi) d \omega .
$$




\section{ACtions of Differentials on modules}

In this section we show how the theory of Leibniz and Kähler differentials is used to study higher order differentials in applications. In a typical situation we have an $A$-module $C$ together with a $k$-linear endomorphism $\varphi$ of $C$ and an element $u$ such that $\varphi(u)=0$. The higher order differentials appear as the elements of the smallest $A$-submodule $\Gamma$ of $C$ that contains $u$ and is invariant under $\varphi$.

Fix an element $\varepsilon$ of $k$. In the above situation we obtain a homomorphism $\Lambda_{A / k}^{\varepsilon} \rightarrow$ $\operatorname{End}_{k}(C)$ of $k$-algebras over $A$, which sends a polynomial $P(t)$ to the endomorphism $P(\varphi)$ of $C$. Denote by $\Lambda^{\varepsilon}(\varphi)$ the image of this map. The map $\operatorname{End}_{k}(C) \rightarrow C$, which sends an endomorphism $\psi$ to $\psi(u)$, induces a surjection $\Lambda^{\varepsilon}(\varphi) \rightarrow \Gamma$ of $A$-modules. The main result of this section gives criteria for the surjection $\Lambda^{\varepsilon}(\varphi) \rightarrow \Gamma$ to be an isomorphism and, when the criteria are fulfilled, gives an explicit formula for the product $\Gamma \otimes_{k} C \rightarrow C$, induced by the natural product $\Lambda^{\varepsilon}(\varphi) \otimes_{k} C \rightarrow C$. In particular, when the criteria are fulfilled, we have that the module $\Gamma$ of higher order differentials inherits a structure as a $k$-algebra from the sub-algebra $\Lambda^{\varepsilon}(\varphi)$ of $\operatorname{End}_{k}(C)$, and we have a surjection $\Lambda_{A / k}^{\varepsilon} \rightarrow \Gamma$ of $k$-algebras over $A$. Moreover, then under the product $\Gamma \otimes_{k} C \rightarrow C$ we have that $C$ is a $\Gamma$-module.

4.1. Remark. Let $C$ be an $A$-module and let $\varphi$ be a $k$-linear endomorphism of $C$. Then the map $P \mapsto P(\varphi)$ is a homomorphism of $k$-algebras over $A$,

$$
\operatorname{Ev}_{\varphi}: A\langle t\rangle \rightarrow \operatorname{End}_{k}(C) .
$$

Accordingly, $C$ has a structure as a module over $A\langle t\rangle$, extending its structure as an $A$-module. The corresponding $A$-linear action,

$$
A\langle t\rangle \otimes_{k} C \rightarrow C
$$

is given by the formula $P . x=P(\varphi)(x)$. The pair $\left(\Delta_{t}^{\varepsilon}, \varphi\right)$ is an $\varepsilon$-derivation for the product (4.1.2). Indeed, since $\Delta_{t}^{\varepsilon} P=t P-\left(\varepsilon_{\mathrm{gr}} P\right) t$, the equation

$$
\varphi(P . x)=\left(\Delta_{t}^{\varepsilon} P\right) \cdot x+\left(\varepsilon_{\mathrm{gr}} P\right) \cdot \varphi(x)
$$

follows from the identity

$$
\varphi P(\varphi)=\varphi P(\varphi)-\left(\varepsilon_{\mathrm{gr}} P\right)(\varphi) \varphi+\left(\varepsilon_{\mathrm{gr}} P\right)(\varphi) \varphi .
$$

Restriction of the homomorphism (4.1.1) to the subalgebra $\Lambda_{A / k}^{\varepsilon}$ of $A\langle t\rangle$ defines a homomorphism of $k$-algebras over $A$,

$$
\Lambda_{A / k}^{\varepsilon} \rightarrow \operatorname{End}_{k}(C) .
$$

Denote by $\Lambda^{\varepsilon}(\varphi)$ the image of the homomorphism (4.1.3). Via the homomorphism (4.1.3), we have that $C$ is a module over $\Lambda_{A / k}^{\varepsilon}$ and is a faithful module over $\Lambda^{\varepsilon}(\varphi)$. The corresponding $A$-linear product

$$
\Lambda_{A / k}^{\varepsilon} \otimes_{k} C \rightarrow C
$$

is given by the formula $Q . x:=Q(\varphi)(x)$ for $Q \in \Lambda_{A / k}^{\varepsilon}$ and $x \in C$. Since $\left(\Delta_{t}^{\varepsilon}, \varphi\right)$ is an $\varepsilon$-derivation for the product (4.1.2), it follows that $\left(d_{t}^{\varepsilon}, \varphi\right)$ is an $\varepsilon$-derivation for the product (4.1.4).

4.2. Lemma. The homomorphism $\Lambda_{A / k}^{\varepsilon} \rightarrow \operatorname{End}_{k}(C)$ of (4.1.3) descends to a homomorphism of $k$-algebras over $A$ from the quotient

$$
\Omega_{A / k}^{\varepsilon} \rightarrow \operatorname{End}_{k}(C),
$$


if and only if the endomorphism $\varphi$ of $C$ is a differential operator of order at most 1.

Proof. The map (4.1.3) is the restriction of $\mathrm{Ev}_{\varphi}$ to the subalgebra $\Lambda_{A / k}^{\varepsilon}$ of $A\langle t\rangle$. Under the isomorphism $\Lambda_{A / k}^{\varepsilon} \rightarrow \Lambda_{A / k}$ of (2.13) we have that $\Omega_{A / k}^{\varepsilon}$ is the quotient of $\Lambda_{A / k}^{\varepsilon}$ modulo the ideal $\mathfrak{A}^{\varepsilon}$ consisting of sums of polynomials of the form

$$
P\left(\Delta_{t}^{\varepsilon}\right)(\rho Q) \text {, }
$$

where $P \in A\langle t\rangle, Q \in \Lambda_{A / k}^{\varepsilon}$, and $\rho=\left(\Delta_{t} b\right) a-a\left(\Delta_{t} b\right)$ for $a, b \in A$. The ideal $\mathfrak{A}^{\varepsilon}$ is homogeneous. So, if $\mathfrak{B}$ is the ideal generated by all homogeneous polynomials in the kernel of $\mathrm{Ev}_{\varphi}$, it follows that the homomorphism $\Lambda_{A / k}^{\varepsilon} \rightarrow \operatorname{End}_{k}(C)$ factors through the quotient, if and only if $\mathfrak{B}$ contains all polynomials of the form (4.2.2).

Assume first that $\mathfrak{B}$ contains all polynomials (4.2.2). Then, in particular, $\rho \in \mathfrak{B}$ and thus $\operatorname{Ev}_{\varphi}(\rho)=0$. Since $\rho=\left(\Delta_{t} b\right) a-a\left(\Delta_{t} b\right)$, it follows that $\operatorname{Ev}_{\varphi}\left(\Delta_{t} b\right) a_{C}=$ $a_{C} \operatorname{Ev}_{\varphi}\left(\Delta_{t} b\right)$. Hence the map $\operatorname{Ev}_{\varphi}\left(\Delta_{t} b\right)$ is $A$-linear for all $b \in A$. $\operatorname{As}_{\operatorname{Ev}} \operatorname{Ev}_{\varphi}\left(\Delta_{t} b\right)=$ $\varphi b_{C}-b_{C} \varphi$, it follows that $\varphi$ is a differential operator of order at most 1 .

Assume conversely that $\varphi$ is a differential operator of order at most 1 . Then, by the above calculation, any polynomial of the form $\rho=\left(\Delta_{t} b\right) a-a\left(\Delta_{t} b\right)$ is contained in the kernel of $\mathrm{Ev}_{\varphi}$, and hence $\rho \in \mathfrak{B}$. The ideal $\mathfrak{B}$ is homogeneous, and therefore $\Delta_{t}^{\varepsilon}$-invariant. Therefore, since $\rho \in \mathfrak{B}$, it follows that every element (4.2.2) belongs to $\mathfrak{B}$.

Hence the equivalence has been proved.

4.3. Definition. Let $C$ be an $A$-module and let $\varphi$ be a $k$-linear endomorphism of $C$. Via the isomorphism $\Lambda_{A / k} \stackrel{\sim}{\longrightarrow} \Lambda_{A / k}^{\varepsilon}$ of (2.13), the product (4.1.4) defines an action of $\Lambda_{A / k}$ on $C$

$$
\Lambda_{A / k} \otimes_{k} C \rightarrow C,
$$

denoted $\pi \otimes_{k} x \mapsto \pi . x$. It is called the $\varepsilon$-action of $\Lambda_{A / k}$ on $C$. Note that the product $\pi . x$ depends on $\varepsilon$. As the map (4.1.3) is a map of $k$-algebras over $A$, it follows that the map (4.3.1) is $A$-linear and that the tensor product over $k$ in the source can be replaced by the tensor product over $A$. In other words, for $a \in A$, we have the equations $a(\pi \cdot x)=(a \pi \cdot x)$ and $(\pi \cdot a) \cdot x=\pi \cdot(a x)$. In addition, since $\left(d_{t}^{\varepsilon}, \varphi\right)$ is an $\varepsilon$-derivation for the product (4.1.4), it follows that $\left(d_{t}, \varphi\right)$ is an $\varepsilon$-derivation for the $\varepsilon$-product, that is, for $\pi \in \Lambda_{A / k}$ and $x \in C$, we have the equation

$$
\varphi(\pi \cdot x)=\left(d_{t} \pi\right) \cdot x+\varepsilon_{\mathrm{gr}}(\pi) \cdot x .
$$

For $\varepsilon=0$, we have that the right $\varepsilon$-multiplication by elements of $A$ is the multiplication given by the structure of $\Lambda_{A / k}$ as an $A$-A-module. Hence we may view the map (4.3.1) as an $A$-linear map

$$
\Lambda_{A / k} \otimes_{A} C \rightarrow C
$$

Moreover, if $\varepsilon=0$, then the second term on the right side of (4.3.2) vanishes when $\pi$ has no components of degree 0 . Hence, for $n \geq 1$, there is a commutative diagram

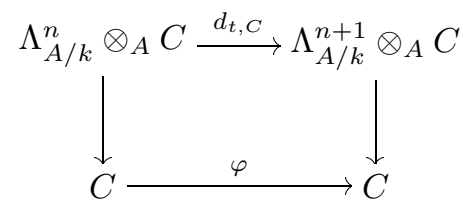

where the vertical maps are the $\varepsilon$-products for $\varepsilon=0$. 
Assume that $\varphi$ is a differential operator of order at most 1 . Then it follows from Lemma (4.2) that the $\varepsilon$-action of $\Lambda_{A / k}$ on $C$ descends to an $\varepsilon$-action of $\Omega_{A / k}$ on $C$. Clearly, the properties described above for the action of $\Lambda_{A / k}$ descend to analogous properties of the action of $\Omega_{A / k}$.

4.4. Proposition. In the setup of (4.1), assume that $C$ is a graded $A$-module, and assume either that $\varphi$ is homogeneous of degree 1 , or assume that $\varepsilon=1$ and that $\varphi$ is homogeneous of degree 0 . Let $u$ be an element of degree 0 in the kernel of $\varphi$, and let $\Gamma=\Lambda(C, \varphi, u)$ be the submodule of $C$ defined in (2.8). Then the following three conditions are equivalent:

(i) The evaluation map $\operatorname{End}_{k}(C) \rightarrow C$ given by $\lambda \mapsto \lambda(u)$ induces an isomorphism $E: \Lambda^{\varepsilon}(\varphi) \stackrel{\sim}{\longrightarrow} \Gamma$.

(ii) If $Q$ is a polynomial in $\Lambda_{A / k}^{\varepsilon}$ such that $Q(\varphi)(u)=0$, then $Q(\varphi)=0$.

(iii) There exists an A-linear product $\Gamma \otimes_{k} C \rightarrow C$ for which $u$ is a left unit and $\varphi$ is an $\varepsilon$-derivation, i.e., $\varphi(\gamma \cdot x)=\varphi(\gamma) \cdot x+\left(\varepsilon_{\mathrm{gr}} \gamma\right) \cdot \varphi(x)$ for $\gamma \in \Gamma$ and $x \in C$.

Assume that the three conditions hold. Then the following assertions hold:

(1) The product in (iii) is unique and given by the formula

$$
P(\varphi)(u) \cdot x=\sum_{i=0}^{\infty}(-1)^{i} \varepsilon^{i(i-1) / 2} \partial_{i}^{\varepsilon} P(\varphi) \varphi^{i}(x) .
$$

(2) The A-submodule $\Gamma$ of $C$ is stable under the product. Moreover, with the induced product, the $A$-module $\Gamma$ is a $k$-algebra over $A$ with $a \mapsto$ au as structure map, and $C$ is a faithful $\Gamma$-module.

Proof. Let $B$ be the image of the map (4.1.1). Then $B$ is a $k$-subalgebra over $A$ of $\operatorname{End}_{k}(C)$. Assume first that $\varphi$ is homogeneous of degree 1 . If $P$ is a polynomial of degree $p$, the image $P(\varphi)$ is homogeneous of degree $p$. Hence the image $B$ has a natural structure of a graded $k$-algebra over $A$ such that the map $A\langle t\rangle \rightarrow B$ is a surjection of graded $k$-algebras over $A$. Let $\Delta_{\varphi}^{\varepsilon}$ be the interior $\varepsilon$-derivation of $B$ determined by $\varphi$. Then, clearly, the map $P \mapsto P(\varphi)$ is a surjection of $k$-algebras over $A$ with endomorphisms

$$
\left(A\langle t\rangle, \Delta_{t}^{\varepsilon}\right) \rightarrow\left(B, \Delta_{\varphi}^{\varepsilon}\right) .
$$

If $\varphi$ is of degree 0, then all endomorphisms of $B$ are of degree 0 . However, then $\varepsilon=1$ and $\Delta_{\varphi}^{\varepsilon}$ is the usual commutator, so we still have the surjection (4.4.2).

The elements of $\Lambda_{A / k}^{\varepsilon}$ are polynomials of the form $P\left(\Delta_{t}^{\varepsilon}\right)(1)$, and under (4.4.2) the polynomial $P\left(\Delta_{t}^{\varepsilon}\right)(1)$ is mapped to the endomorphism $P\left(\Delta_{\varphi}^{\varepsilon}\right)\left(1_{C}\right)$. It follows that the $k$-subalgebra $\Lambda^{\varepsilon}(\varphi)$ of $B$ consists of the endomorphisms of the form $P\left(\Delta_{\varphi}^{\varepsilon}\right)\left(1_{C}\right)$. In particular, $\Lambda^{\varepsilon}(\varphi)$ is invariant under $\Delta_{\varphi}^{\varepsilon}$. Denote by $d_{\varphi}^{\varepsilon}$ the induced $\varepsilon$-derivation of $\Lambda^{\varepsilon}(\varphi)$. Clearly, the pair $\left(d^{\varepsilon}, \varphi\right)$ is an $\varepsilon$-derivation for the product $\Lambda^{\varepsilon}(\varphi) \otimes_{k} C \rightarrow C$.

Since $\varphi(u)=0$, it follows that evaluation $\lambda \mapsto \lambda(u)$ is a map $\left(B, \Delta_{\varphi}^{\varepsilon}\right) \rightarrow(C, \varphi)$. Hence, under evaluation, $P\left(\Delta_{\varphi}^{\varepsilon}\right)\left(1_{C}\right)$ is mapped to $P(\varphi)(u)$. Therefore, the evaluation map is a surjection of $A$-modules with $k$-linear endomorphisms

$$
E:\left(\Lambda^{\varepsilon}(\varphi), d_{\varphi}^{\varepsilon}\right) \rightarrow(\Gamma, \varphi) .
$$

Clearly, the condition (ii) is that the homomorphism $E$ is injective. Therefore, the conditions (i) and (ii) are equivalent. 
Assume that the conditions (i) and (ii) hold. Then an $A$-linear product as in (iii) is defined using the structure of $C$ as a $\Lambda^{\varepsilon}(\varphi)$-module and the isomorphism $E$. The element $u$ is a unit for the product, because $u=E\left(1_{C}\right)$ is the image of the unit $\Lambda^{\varepsilon}(\varphi)$. It follows from (4.4.3) that the endomorphism $\varphi$ of $\Gamma$ under the isomorphism $E$ corresponds to the endomorphism $d_{\varphi}$ of $\Lambda^{\varepsilon}(\varphi)$. Therefore, since $\left(d^{\varepsilon}, \varphi\right)$ is an $\varepsilon$ derivation, it follows that $\varphi$ is a derivation for the product $\Gamma \otimes_{k} C \rightarrow C$.

Conversely, assume that there is given a product with the properties in (iii). It follows from the second equation of Corollary (1.9) that the equation (4.4.1) holds. In particular, for $Q \in \Lambda_{A / k}^{\varepsilon}$ we have the equation

$$
Q(\varphi)(u) \cdot x=Q(\varphi)(x) .
$$

It follows from the latter equation that the composition

$$
\Lambda^{\varepsilon}(\varphi) \stackrel{E}{\longrightarrow} \Gamma \rightarrow \operatorname{End}_{k}(C),
$$

where the second map is the representation determined by the product $\Gamma \otimes_{k} C \rightarrow C$, is equal to the inclusion of $\Lambda^{\varepsilon}(\varphi)$ in $\operatorname{End}_{k}(C)$. In particular, the homomorphism $E$ is injective, that is, condition (ii) holds.

Thus the three conditions are equivalent. To prove the remaining assertions, assume that the three conditions hold. The equation (4.4.1) was shown to be a consequence of the properties of the product. Clearly, the equation implies that the product is unique. Moreover, the equation (4.4.1) implies the equation (4.4.4) for $Q \in \Lambda_{A / k}^{\varepsilon}$. Since $E$ is surjective, any element $x$ of $\Gamma$ is of the form $R(\varphi)(u)$ for a polynomial $R$ of $\Lambda_{A / k}^{\varepsilon}$. It follows from (4.4.4) that

$$
Q(\varphi)(u) \cdot R(\varphi)(u)=Q(\varphi) R(\varphi)(u) .
$$

Therefore, the $A$-module $\Gamma$ is stable under the product and the map $E$ is a homomorphism also with respect to the product. Since $\Lambda^{\varepsilon}(\varphi)$ is a $k$-algebra over $A$ and $C$ is a $\Lambda^{\varepsilon}(\varphi)$-module, it follows that $\Gamma$ is a $k$-algebra over $A$ and that $C$ is a $\Gamma$-module. Thus the remaining assertions of the proposition have been proved.

4.5. Note. Let $\rho:=\left(\Delta_{t} b\right) a-a\left(\Delta_{t} b\right)$ with $a, b \in A$. As noted in the proof of (4.2), we have that $\rho(\varphi)=\varphi b a-b \varphi a-a \varphi b+a b \varphi$. In particular, if $u \in C$ is an element in the kernel of $\varphi$, then we have the equation

$$
\rho(\varphi)(u)=\varphi(b a u)-b \varphi(a u)-a \varphi(b u) .
$$

Assume that $A$ is commutative. Then, clearly, we have that $\rho(\varphi)(u)=0$ for all $a, b \in A$, if and only if the map $a \mapsto \varphi(a u)$ is a module derivation $A \rightarrow C$. In particular, in the setup of (4.4), if the condition (ii) holds and $a \mapsto \varphi(a u)$ is a module derivation, then $\varphi$ is a differential operator of order at most 1 .

\section{DeRivation SYSTEMS}

The natural framework for the theory of higher order differentials is derivation systems. Such systems consist of a graded $A$-module $C$ such that the graded piece $C_{0}$ of degree 0 is isomorphic to $A$, together with a differential operator $d$ on $C$ of order at most 1 such that $d(1)=0$. The derivation systems give rise to triples $(C, \varphi, u)$ of the form considered in the previous section, with $\varphi:=d$ and $u:=1$. In applications all such triples come from derivation systems. The higher order differentials of the derivation system are the elements of the smallest $d$-invariant 
$A$-submodule $\Omega(C)$ of $C$ which contains $1 \in A=C_{0}$. We have that $\Omega(C)$ is a graded $A$ module.

Since $d$ is a differential operator of order at most 1 , we obtain by Lemma (3.4) an $A$-linear map $\Omega_{A / k}^{1} \otimes_{A} C \rightarrow C$, denoted $\rho \otimes_{A} F \mapsto \rho . F$, such that $d f . F=$ $d(f F)-f d F$, for all $f \in A$ and $F \in C$. Moreover, since in addition $d(1)=0$, we have that $d: A \rightarrow C_{1}$ is an $A$-module derivation and we obtain a surjective $A$-linear map $\Omega_{A / k}^{1} \rightarrow \Omega^{1}(C)$. In all applications, the map $\Omega_{A / k}^{1} \otimes_{A} C \rightarrow C$ factors over the quotient map $\Omega_{A / k}^{1} \otimes_{A} C \rightarrow \Omega^{1}(C) \otimes_{A} C$. When the latter condition is satisfied, we call the derivation system $(C, d)$ a Kähler system.

The main part of this section is devoted to the examples that appear most frequently in applications, and to illustrate how our setup is designed to incorporate, in a natural way, the main applications. In particular, we introduce the system of higher order differentials for $C^{\infty}$-manifolds constructed by P.-A. Meyer, and our non-commutative generalization of Iitaka's higher order differentials for smooth schemes. Both systems are Kähler systems.

5.1. Setup. Assume that $A$ is a commutative $k$-algebra. In the sequel we will consider systems $(C, d)$ consisting of a graded $A$-module $C$ whose component of degree 0 is equal to $A$ and a $k$-linear endomorphism $d$ of degree 1 of $C$ such that the unity 1 of $A$ is in the kernel of $d$. The system will be called a derivation system for the $k$-algebra $A$, if $d$ is a differential operator of order at most 1 . To distinguish the endomorphism $d$ of $C$ from the endomorphism of the module $\Omega_{A / k}$ of Kähler differentials, we write $d_{A / k}$ for the latter.

Fix a derivation system $(C, d)$. The homogeneous components of the map $d$ may be viewed as a sequence of differential operators of order at most 1 :

$$
A=C_{0} \stackrel{d}{\rightarrow} C_{1} \stackrel{d}{\rightarrow} C_{2} \stackrel{d}{\rightarrow} \cdots .
$$

Clearly, since 1 is in the kernel of $d$, the component $d: A \rightarrow C_{1}$ is a module derivation. Denote by $\Omega(C)$ the smallest $d$-invariant $A$-submodule of $C$ containing the unity 1 . In the notation of (2.8) we have that $\Omega(C)=\Lambda(C, d, 1)$ is the image of the map $A\langle t\rangle \rightarrow C$ given by $P \mapsto P(d)(1)$. Clearly, $\Omega(C)$ is a homogeneous $A$-submodule of $C$. Its elements of degree $n$ will be called $n$-forms. The 0 -forms are the elements of $A=C_{0}$ and the 1-forms are $A$-linear combinations of elements of the form $d a$ for $a \in A$.

Since the unity 1 is in the kernel of $d$, the map $P \mapsto P(d)(1)$ induces by $(2.8)$ a map of graded $A$-modules with $k$-linear endomorphisms

$$
\left(\Lambda_{A / k}, d_{t}\right) \rightarrow(C, d) .
$$

The image of $\pi \in \Lambda_{A / k}$ will be denoted by $\bar{\pi}$.

Since $d$ is a differential operator of order at most 1, there is, by Lemma (3.4), an $A$-linear product $\Omega_{A / k}^{1} \otimes_{A} C \rightarrow C$, denoted $\rho \otimes_{A} x \mapsto \rho . x$, such that, for all $f \in A$ and $F \in C$, we have that

$$
d(f F)=\left(d_{A / k} f\right) \cdot F+f d F .
$$

The $k$-linear map of degree -1 obtained as in Lemma (2.5) by taking as $w$ the $k$-linear map $-d: A \rightarrow \Omega^{1}(C)$ will be denoted

$$
s_{C, t}: \Lambda_{A / k} \rightarrow \Omega^{1}(C) \otimes_{A} \Lambda_{A / k} .
$$

Obviously, the map $s_{C, t}$ is the composition of the $k$-linear map $s_{t}$ of (2.6) and the surjective $A$-linear map $\Lambda_{A / k}^{1} \otimes_{A} \Lambda_{A / k} \rightarrow \Omega^{1}(C) \otimes_{A} \Lambda_{A / k}$ induced by the canonical 
map (5.1.1). In particular, for $f \in A, \rho \in \Lambda_{A / k}^{1}$, and $\pi \in \Lambda_{A / k}^{n}$, we obtain from (2.6.2) the formulas

$$
s_{C, t}\left(f d_{t} \pi\right)=-d f \otimes_{A} \pi, \quad s_{C, t}(\rho . \pi)=\bar{\rho} \otimes_{A} \pi .
$$

When $\Lambda_{A / k}$ is identified with the subalgebra $\Lambda_{A / k}^{\varepsilon}$ of $A\langle t\rangle$ as in (2.13.1), we write

$$
s_{C, t}^{\varepsilon}: \Lambda_{A / k}^{\varepsilon} \rightarrow \Omega^{1}(C) \otimes_{A} \Lambda_{A / k}^{\varepsilon}
$$

for the resulting map.

Since $d$ is a differential operator of order at most 1, it follows from (3.11) that the map (5.1.1) induces a homomorphism from the quotient

$$
\left(\Omega_{A / k}, d_{A / k}\right) \rightarrow(C, d),
$$

and $\Omega(C)$ is the image of the induced map. Equivalently, there is a commutative diagram with $A$-linear vertical maps,

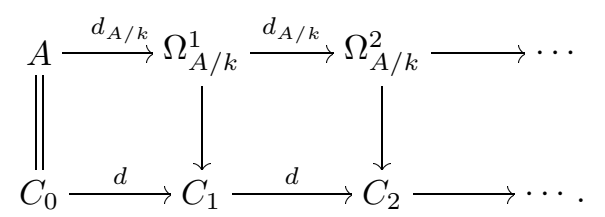

5.2. Definition. A derivation system $(C, d)$ as in (5.1) will be called a Kähler system is there exists an $A$-linear product $\Omega^{1}(C) \otimes_{A} C \rightarrow C$, denoted $\rho \otimes_{A} F \mapsto \rho . F$, such that, for $f \in A$ and $F \in C$, the following equation holds:

$$
d f . F=d(f F)-f d F .
$$

Clearly, the product is completely determined by the equation (5.2.1). Equivalently, the system $(C, d)$ is a Kähler system if the product $\Omega_{A / k}^{1} \otimes_{A} C \rightarrow C$ determined by (5.1.2) factors over the quotient $\Omega^{1}(C) \otimes_{A} C$. In particular, if the canonical surjection $\Omega_{A / k}^{1} \rightarrow \Omega^{1}(C)$ of (5.1.7) is an isomorphism, then the system is a Kähler system.

Note that if a system $(C, d)$ as in $(5.1)$ is given such that there exists a product satisfying the equation (5.2.1), then $d$ is a differential operator of order at most 1 .

If $(C, d)$ is a Kähler system, then, for $\rho \in \Lambda_{A / k}^{1}$ and $\pi \in \Lambda_{A / k}$, we have the following equation:

$$
\overline{\rho . \pi}=\bar{\rho} . \bar{\pi} .
$$

Indeed, to verify the equation, we may assume that $\rho=d_{t} f$ for $f \in A$. Then, by (2.4.2), we have that $d_{t} f \cdot \pi=d_{t}(f \pi)-f d_{t} \pi$. It follows that

$$
\overline{d_{t} f \cdot \pi}=d(f \bar{\pi})-f d \bar{\pi}=d f \cdot \bar{\pi}=\overline{d_{t} f} \cdot \bar{\pi} .
$$

5.3. Example. Let $d: A \rightarrow M$ be a module derivation. Then, clearly, there is a derivation system $(C, d)$ with $C_{0}:=A, C_{1}:=M$ and $C_{n}:=0$ for $n>1$. The system is a Kähler system with the product $\Omega^{1}(C) \otimes_{A} C_{n} \rightarrow C_{n+1}$ defined as the inclusion of $\Omega^{1}(C)$ into $C_{1}$ for $n=0$ and as the zero map for $n>0$.

5.4. Example. Assume that $C$ is a graded $k$-algebra over $A$ with $C_{0}=A$. Let $D$ be an $\varepsilon$-derivation of degree 1 of $C$. Assume for all $f \in A$ that the element $D f$ in $C_{1}$ commutes with the elements of $A$. Then $(C, D)$ is a Kähler system. Indeed, since $D$ is an $\varepsilon$-derivation and the elements of $A$ are of degree 0 , we have that $D(f x)-f D(x)=D(f) x$. Hence the endomorphism $D$ is a differential operator of 
degree at most 1 , and the product of (5.2) is given by the product in the algebra C.

5.5. Example. Clearly, the free Kähler differentials form a derivation system $(C, d)=\left(\Omega_{A / k}, d_{A / k}\right)$. Obviously, $\Omega^{1}(C)=\Omega_{A / k}^{1}$, and hence the system is a Kähler system. Moreover, as noted at the end of (5.1), the system of free Kähler differentials is the universal derivation system for $A$.

An alternative construction of the universal derivation system is given as follows: Let $d: C_{0} \rightarrow C_{1}$ be the map $d_{A / k}: A \rightarrow \Omega_{A / k}^{1}$. Inductively, for $n \geq 1$, let $C_{n+1}$ be the $A$-module $P_{A / k}^{1}\left(C_{n}\right)$ of first order principal parts of $C_{n}$, and let $d=d_{C_{n}}^{1}$ : $C_{n} \rightarrow C_{n+1}$ be the universal differential operator of order at most 1 defined on $C_{n}$. Then, clearly, the system $(C, d)$ is a universal derivation system. It follows that the system obtained by the alternative construction is isomorphic to the system of free Kähler differentials. In particular, there is, for $n \geq 1$, an $A$-linear isomorphism

$$
\Omega_{A / k}^{n+1} \stackrel{\sim}{\longrightarrow} \overbrace{P_{A / k}^{1} \cdots P_{A / k}^{1}}^{n}\left(\Omega_{A / k}^{1}\right) .
$$

5.6. Remark. Several important derivation systems are obtained as follows:

We are given a sequence of maps of commutative $k$-algebras

$$
A=C_{0} \stackrel{\iota}{\rightarrow} C_{1} \stackrel{\iota}{\rightarrow} C_{2} \stackrel{\iota}{\rightarrow} \cdots \text {. }
$$

View each $C_{n+1}$ as a $C_{n}$-module via the map $\iota$. In addition, assume that we have $k$-linear maps $d: C_{n} \rightarrow C_{n+1}$ which are module derivations from the $k$-algebra $C_{n}$ to the $C_{n}$-module $C_{n+1}$.

The structure on $C_{n}$ as an $A$-module is given by the composition $\iota^{n}: A \rightarrow C_{n}$, that is, by $f F:=\left(\iota^{n} f\right) F$ for $f \in A$ and $F \in C_{n}$. Since each map $d: C_{n} \rightarrow C_{n+1}$ is a module derivation, we have that

$$
d(F G)=(d F)(\iota G)+(\iota F)(d G) .
$$

As a consequence, for $f \in A$ and $F \in C_{n}$, we have that

$$
d(f F)-f d F=\left(d \iota^{n} f\right)(\iota F) .
$$

In particular, it follows that each map $d: C_{n} \rightarrow C_{n+1}$ is a differential operator of order at most 1 for $A$. Hence the system $(C, d)$ is a derivation system, and the product $\Omega_{A / k}^{1} \otimes_{A} C \rightarrow C$ is determined by the equation

$$
\left(d_{A / k} f\right) \cdot F=\left(d \iota^{n} f\right)(\iota F)
$$

for $a \in A$ and $F \in C_{n}$. Moreover, the system is a Kähler system if and only if, for each $n \geq 1$, there exists an $A$-linear map $i_{n}: \Omega^{1}(C) \rightarrow C_{n+1}$ such that, for $f \in A$, we have that

$$
i_{n}(d f)=d \iota^{n} f .
$$

Indeed, if the product of (5.2) exists, then $i_{n}$ may be defined by $i_{n}(\rho)=\rho .1_{n}$ where $1_{n}$ is the unity of $C_{n}$. Conversely, if the maps $i_{n}$ exist, then the product may be defined by $\rho . F=i_{n}(\rho) \iota F$.

For instance, there is a derivation system $\left(C_{A / k}, d\right)$ defined as follows: Let $C_{0}:=$ $A$ and, inductively, let $C_{n+1}=\operatorname{Sym}_{C_{n}} \Omega_{C_{n} / k}^{1}$ be the symmetric algebra of the $C_{n^{-}}$ module $\Omega_{C_{n} / k}^{1}$ and let $d: C_{n} \rightarrow C_{n+1}$ be the composition of the universal derivation $d_{C_{n} / k}: C_{n} \rightarrow \Omega_{C_{n} / k}^{1}$ and the inclusion of $\Omega_{C_{n} / k}^{1}$ into $C_{n+1}=\operatorname{Sym}_{C_{n}} \Omega_{C_{n} / k}^{1}$. The system is a Kähler system, because $\Omega_{A / k}^{1}=\Omega^{1}(C)$. 
This example is the non-commutative version of Iitaka's construction referred to in the introduction.

5.7. Example (Iitaka). Consider the following construction: Given commutative $k$-algebras $A=C_{0}$ and $C_{1}$ and maps $\iota, d: C_{0} \rightarrow C_{1}$, where $\iota$ is a map of $k$-algebras and $d$ is a module derivation from $C_{0}$ to the $C_{0}$-module $C_{1}$. Form the symmetric algebra $S:=\operatorname{Sym}_{C_{1}} \Omega_{C_{1} / k}^{1}$, and the maps $\tilde{\iota}, \tilde{d}: C_{1} \rightarrow S$, where $\tilde{\iota}$ is the inclusion of algebras $C_{1} \rightarrow S$ and $\tilde{d}$ is the universal derivation $d_{C_{1} / k}: C_{1} \rightarrow S$. Finally, let $C_{2}$ be the $k$-algebra obtained as the quotient of $S$ modulo the ideal generated by all differences $\tilde{\iota} d(x)-\tilde{d} \iota(x)$ for $x \in C_{0}$. Define new maps $\iota, d: C_{1} \rightarrow C_{2}$ as the maps obtained by composing $\tilde{\iota}, \tilde{d}$ with the quotient map $S \rightarrow C_{2}$.

Clearly, the $k$-algebra $C_{2}$ and the new maps $\iota, d: C_{1} \rightarrow C_{2}$ have the following universal property: Given commutative $k$-algebras $C_{0}^{\prime}, C_{1}^{\prime}, C_{2}^{\prime}$ and maps $\iota^{\prime}, d^{\prime}$ : $C_{0}^{\prime} \rightarrow C_{1}^{\prime}$ and $\iota^{\prime}, d^{\prime}: C_{1}^{\prime} \rightarrow C_{2}^{\prime}$ such that the maps $\iota^{\prime}$ are maps of $k$-algebras and the maps $d^{\prime}$ are module derivations. Let $\psi_{0}: C_{0} \rightarrow C_{0}^{\prime}$ and $\psi_{1}: C_{1} \rightarrow C_{1}^{\prime}$ be maps of $k$-algebras such that $\psi_{1} \iota=\iota^{\prime} \psi_{0}$ and $\psi_{1} d=d^{\prime} \psi_{0}$. Assume that $d^{\prime} \iota^{\prime}=\iota^{\prime} d^{\prime}$. Then there is a unique map of $k$-algebras $\psi_{2}: C_{2} \rightarrow C_{2}^{\prime}$ such that $\psi_{2} \iota=\iota^{\prime} \psi_{1}$ and $\psi_{2} d=d^{\prime} \psi_{1}$.

The construction is iterated as follows: Assume that $\iota, d: C_{n-1} \rightarrow C_{n}$ are defined, where $\iota$ is a map of $k$-algebras and $d$ is a module derivation into the $C_{n-1}$ module $C_{n}$. Apply the construction with $C_{0}:=C_{n-1}$ and $C_{1}:=C_{n}$ to obtain a $k$-algebra $C_{n+1}$ and maps $\iota, d: C_{n} \rightarrow C_{n+1}$.

The system obtained by the iterated construction is a Kähler system. Indeed, for $n \geq 1$, let $Q_{n}$ denote the $C_{n-1}$-submodule of $C_{n}$ generated by elements $d x$ for $x \in C_{n-1}$. In particular, then $Q_{1}=\Omega^{1}(C)$. It follows from the construction that we have the equation $\iota d=d \iota$ of composite maps $C_{n-1} \rightarrow C_{n+1}$. In particular, the $C_{n}$-linear map $\iota: C_{n} \rightarrow C_{n+1}$ induces a $C_{n-1}$-linear map of submodules $i: Q_{n} \rightarrow Q_{n+1}$. Clearly, the following diagram is commutative:

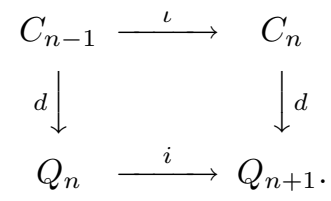

It follows that if $i_{n}: \Omega^{1}(C) \rightarrow C_{n+1}$ is the map defined by $n$ compositions of the maps $i$ followed by the inclusion of $Q_{n+1}$ into $C_{n+1}$, then $i_{n}(d f)=d \iota^{n} f$. Therefore, as noted in (5.6), the system are a Kähler system.

The derivation system $\left(C^{\mathrm{Iit}}, d\right)$, related to the works of Iitaka [10], [11], [12], [13] and Johnson [14], [15], is the system obtained when $C_{0}=A, C_{1}:=\operatorname{Sym}_{A} \Omega_{A / k}^{1}$ and $\iota, d: A \rightarrow C_{1}$ are, respectively, the inclusion of algebras and the universal derivation $d_{A / k}$. In particular, the system of Iitaka is a Kähler system. Moreover, by the universal property of the simple construction of the first paragraph, we obtain the universal property of the system $\left(C^{\mathrm{Iit}}, d\right)$ : Given a derivation system $(C, d)$ with the additional structure of (5.6). Assume that $d \iota=\iota d$, that is, the system is a prolongation sequence in the sense of Johnson [15]. Then any map $\psi_{0}: A \rightarrow C_{0}$ of $k$-algebras extends uniquely to a family $\psi=\left\{\psi_{n}\right\}$ of maps of $k$-algebras $\psi_{n}: C_{n}^{\mathrm{Iit}} \rightarrow C_{n}$ such that $\psi d=d \psi$ and $\psi \iota=\iota \psi$.

Let $\Omega_{A / k}^{\mathrm{Iit}}$ be the $k$-algebra obtained as the direct limit of the $k$-algebras $C_{n}^{\text {Iit }}$ with respect to the maps $\iota$. Then $\Omega_{A / k}^{\mathrm{Iit}}$ is a commutative $A$-algebra. Moreover, since 
$d \iota=\iota d$, the derivations $d$ induce a derivation $d$ of $\Omega_{A / k}^{\mathrm{Iit}}$. Let $B$ be a commutative $A$-algebra, and let $D$ be a $k$-linear derivation of $B$. By the universal property of the system $\left(C^{\mathrm{Iit}}, d\right)$, applied to the system $(C, d)$ where $C_{n}:=B, d:=D$ and the maps $\iota$ are the identity of $B$, there is a unique map of $k$-algebras with $k$-linear derivations

$$
\left(\Omega_{A / k}^{\mathrm{Iit}}, d\right) \rightarrow(B, D) .
$$

Consider the $A$-module $\Omega_{A / k}$ with the $\varepsilon$-product of (3.10) for $\varepsilon=1$. Then $\Omega_{A / k}$ is a $k$-algebra over $A$, and $d_{A / k}$ is a derivation of the algebra. Let $\Omega_{A / k}^{\text {sym }}$ be the quotient algebra obtained by dividing the algebra $\Omega_{A / k}$ by its commutator ideal. Then $\Omega_{A / k}^{\mathrm{sym}}$ is a commutative $A$-algebra and it has a derivation $d$ induced by $d_{A / k}$. By the universal property (3.11) of $\left(\Omega_{A / k}, d_{A / k}\right)$, applied when $\Gamma:=B$ is a commutative $k$-algebra and $D$ is a derivation, it follows that $\left(\Omega_{A / k}^{\mathrm{sym}}, d\right)$ has the same universal property as $\left(\Omega_{A / k}^{\mathrm{Iit}}, d\right)$. Hence there is a canonical isomorphism of $\Omega_{A / k}^{\mathrm{sym}}$ and $\Omega_{A / k}^{\mathrm{Iit}}$. The algebra $\Omega_{A / k}^{\mathrm{sym}}$ and related algebras will be studied in more detail in Section 7 .

5.8. Before we introduce the next example, we give some properties of the module of first order Kähler differentials of a symmetric algebra. The setup will be used in Example (5.9), and is analogous to the setup in Example (5.10) on differentiable manifolds.

Let $Q$ be an $A$-module. Form the symmetric algebra $S:=\operatorname{Sym}_{A} Q$ and the universal derivation $d_{S / k}: S \rightarrow \Omega_{S / k}^{1}$. Then, as is well known, there is a canonical isomorphism $\Omega_{S / A}^{1}=Q \otimes_{A} S$ of $S$-modules and a commutative diagram

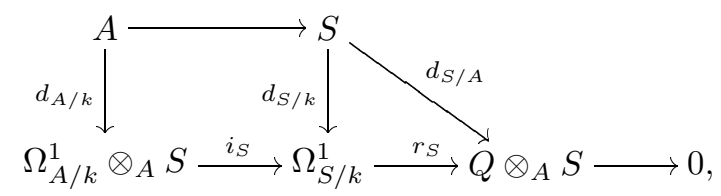

where the bottom row is the usual exact sequence of $S$-linear maps induced from the composition $k \rightarrow A \rightarrow S$. In fact, since $S$ is a graded algebra, the modules $\Omega_{S / k}^{1}$ and $\Omega_{S / A}^{1}$ are graded $S$-modules, and the maps of the diagram are homogeneous, when the factor $Q$ in the tensor product is given degree 1 .

Denote by $\Omega_{S / k}^{1,1}$ the component of degree 1 of $\Omega_{S / k}^{1}$. Then the degree- 1 part of the diagram (5.8.1) is a commutative diagram

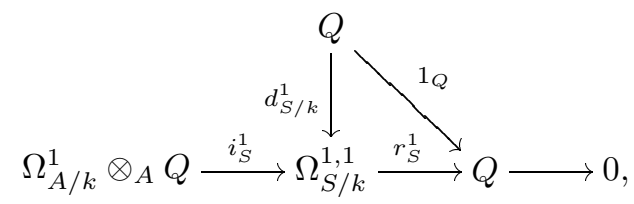

where the row is an exact sequence of $A$-linear maps.

It is a consequence of the following lemma that the map $i_{S}^{1}$ is always injective.

Lemma. The map $i_{S}^{1}$ of (5.8.2) is always injective. In fact, there is an A-linear isomorphism $\Omega_{S / k}^{1,1}=P_{A / k}^{1}(Q)$ under which the row in (5.8.2) is isomorphic to the short exact sequence of (3.3.4). Moreover, the following conditions are equivalent:

(i) The homomorphism $i_{S}^{1}$ in (5.8.2) has an A-linear left-inverse.

(ii) There exists a k-linear connection $\nabla: Q \rightarrow \Omega_{A / k}^{1} \otimes_{A} Q$. 
(iii) The homomorphism $i_{S}$ in (5.8.1) has a homogeneous $S$-linear left-inverse.

(iv) The homomorphism $i_{S}$ in (5.8.1) has an S-linear left-inverse.

Proof. To prove the first part of the lemma, view $\Omega_{S / k}^{1}$ as the quotient of $S \otimes_{k} S$ modulo the left $S$-submodule generated by all tensors $P \otimes_{k} Q+Q \otimes_{k} P-1 \otimes_{k} P Q$ for $P, Q \in S$. The part of degree 1 of $S \otimes_{k} S$ is then the direct sum

$$
\left(A \otimes_{k} Q\right) \oplus\left(Q \otimes_{k} A\right)
$$

and $\Omega_{S / k}^{1,1}$ is the quotient of the direct sum modulo the $A$-submodule generated by all tensors

$$
q a \otimes_{k} b+q b \otimes_{k} a-q \otimes_{k} a b, \quad a \otimes_{k} q+q \otimes_{k} a-1 \otimes_{k} a q,
$$

for $q \in Q, a, b \in A$. Under this identification, the $k$-linear map $d_{S / k}^{1}: Q \rightarrow \Omega_{S / k}^{1,1}$ maps the element $q$ of $Q$ to the class represented by the tensor $1 \otimes_{k} q$.

By the construction of (3.3), there is a similar description of $P_{A / k}^{1}(Q)$ as the quotient of $A \otimes_{k} Q$ modulo the $A$-submodule generated by all tensors

$$
1 \otimes_{k} b a q-b \otimes_{k} a q-a \otimes_{k} b q+a b \otimes_{k} q
$$

for $q \in Q, a, b \in A$. The map $d_{Q}^{1}: Q \rightarrow P_{A / k}^{1}(Q)$ maps the element $q$ of $Q$ to the class represented by $1 \otimes_{k} q$.

Let $u: A \otimes_{k} Q \rightarrow\left(A \otimes_{k} Q\right) \oplus\left(Q \otimes_{k} A\right)$ be the $A$-linear inclusion of $A \otimes_{k} Q$ into the first summand of the direct sum, and let $v:\left(A \otimes_{k} Q\right) \oplus\left(Q \otimes_{k} A\right) \rightarrow A \otimes_{k} Q$ be the $k$-linear map defined by

$$
v\left(a \otimes_{k} q\right):=a \otimes_{k} q, \quad v\left(q \otimes_{k} a\right):=1 \otimes_{k} a q-a \otimes_{k} q .
$$

Under $u$, the element $(* *)$ is mapped into the $A$-submodule generated by elements of the second type in $(*)$. Under $v$, the element of the first type in $(*)$ is mapped to the element $(* *)$ and the element of the second type in $\left(^{*}\right)$ is mapped to zero. Therefore, the maps $u$ and $v$ induce maps of the quotients. It is easily verified that the $A$-linear map induced by $u$ is the inverse of the map induced by $v$. Moreover, under the isomorphism, the maps $i_{Q}^{1}$ and $r_{Q}^{1}$ of (3.3.4) correspond, respectively, to the maps $i_{S}^{1}$ and $r_{S}^{1}$ of (5.8.2). Hence the first part of the lemma has been proved.

Consider the four conditions. Clearly, (iii) implies (iv). To see that (iv) implies (i), compose an $S$-linear retraction for $i_{S}$ with the $A$-linear projection on the degree 1-part. To see that (i) implies (ii), let $w$ be an $A$-linear retraction for $i_{S}^{1}$, define $\nabla:=w d_{S / k}^{1}$ and check that $\nabla$ is a connection.

It remains to prove that (ii) implies (iii). For convenience, set $N:=\Omega_{A / k}^{1} \otimes_{A} S$. To define an $S$-linear map $\Omega_{S / k}^{1} \rightarrow N$, we have to define a $k$-linear derivation $D: S \rightarrow N$. The derivation is defined as follows: Consider the $k$-algebra $S \oplus N$, where $N$ is viewed as an ideal of square zero. The map $a \mapsto a+\left(d_{A / k} a \otimes_{A} 1\right)$ is easily seen to be a map of $k$-algebras

$$
A \rightarrow S \oplus N .
$$

Moreover, the map $q \mapsto q+\nabla(q)$ is an $A$-linear map $Q \rightarrow S \oplus N$. The latter map extends to a homomorphism of $A$-algebras

$$
S=\operatorname{Sym}_{A} Q \rightarrow S \oplus N .
$$

Clearly, the extended homomorphism has the form $P \mapsto P+D(P)$, where $D: S \rightarrow$ $N$ is a $k$-linear derivation. Moreover, for $a \in A$ we have that $D(a)=d_{A / k} a \otimes_{A} 1$. 
Therefore, the derivation $D$ induces an $S$-linear retraction for the map $i_{S}$. Moreover, since $D$ is homogeneous, the retraction is homogeneous. Thus (iii) holds.

Thus the equivalence of the four conditions has been proved.

Note that the equivalent conditions of the lemma hold when the $A$-module $Q$ is induced from a $k$-module $V$. Indeed, if $Q=A \otimes_{k} V$, then $\Omega_{A / k}^{1} \otimes_{A} Q=\Omega_{A / k}^{1} \otimes_{k} V$, and the map $d_{A / k} \otimes_{k} 1_{V}$ is a connection. In particular, the equivalent conditions hold when the $A$-module $Q$ is free. Moreover, if the $A$-modules $Q$ and $\Omega_{A / k}^{1}$ are free, then it follows from the split exact sequence in (5.8.1) that the $S$-module $\Omega_{S / k}^{1}$ is free.

5.9. Example (Affine analogue of the Semple bundle). Consider the following construction: Let $\kappa: \Omega_{A / k}^{1} \rightarrow Q$ be a given $A$-linear map. Form the symmetric algebra $\tilde{A}:=\operatorname{Sym}_{A} Q$. Let $\iota: A \rightarrow \tilde{A}$ be the inclusion of algebras, and let $d: A \rightarrow \tilde{A}$ be the composition of $\kappa d_{A / k}$ and the inclusion of $Q$ into $\tilde{A}$. Finally, let $\tilde{\kappa}: \Omega_{\tilde{A} / k}^{1} \rightarrow \tilde{Q}$ be the $\tilde{A}$-linear map obtained as the push forward of the map $\kappa \otimes_{A} 1: \Omega_{A / k}^{1} \otimes_{A} \tilde{A} \rightarrow Q \otimes_{A} \tilde{A}$ along the map $i_{\tilde{A}}$ of (5.8.1). The push forward preserves the cokernel. Hence, from the exact sequence (5.8.1) we obtain a commutative diagram of $\tilde{A}$-modules with exact rows:

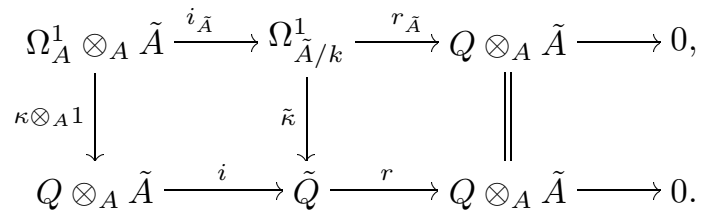

Moreover, as noted at the end of (5.8), if the $A$-module $Q$ is free, then the $\tilde{A}$-linear map $i$ in (5.9.1) is split injective and, consequently, the $\tilde{A}$-module $\tilde{Q}$ is free.

A derivation system $\left(C_{A / k, \kappa}, d\right)$ is obtained from the construction as follows: Set $C_{0}:=A, Q_{1}:=Q$ and let $\kappa: C_{0} \rightarrow Q_{1}$ be the given $A$-linear map. Inductively, assume that the $k$-algebra $C_{n-1}$ and the $C_{n-1}$-linear map $\kappa: \Omega_{C_{n-1} / k} \rightarrow Q_{n}$ are defined. Apply the construction with $A:=C_{n-1}$ and $Q:=Q_{n}$ to obtain a $k$-algebra $C_{n}:=\tilde{C}_{n-1}$ and maps $\iota, d: C_{n-1} \rightarrow C_{n}$ and a $C_{n}$-linear map $\Omega_{C_{n} / k}^{1} \rightarrow Q_{n}$ where $Q_{n+1}:=\tilde{Q}_{n}$.

It follows from the construction that $Q_{n+1} \subseteq C_{n+1}$ and that the image of the map $d: C_{n} \rightarrow C_{n+1}$ is contained in $Q_{n+1}$. Moreover, there is a commutative diagram of $C_{n}$-linear maps,

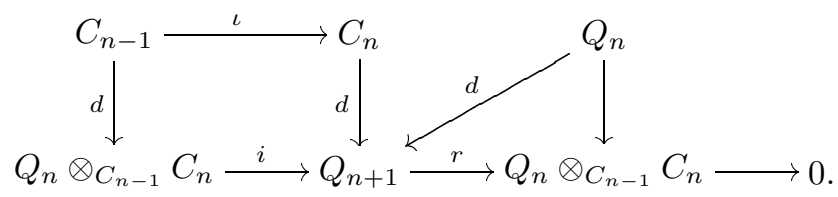

The bottom row is exact, and split exact if $Q$ is a free $A$-module.

It follows that the system is a Kähler system, since compositions of the maps $i$ of (5.9.2) may be used as the $A$-linear maps $i_{n}$ of (5.6).

In the case when $Q$ is a quotient of $\Omega_{A / k}^{1}$ and $\kappa$ is the quotient map, the construction of the module $\tilde{Q}$ is the affine analogue of the Semple bundle used to study 
higher tangencies of varieties by Collino [7], Colley-Kennedy [5], [6], and ArrondoSols-Speiser [24]. In this case, we write $\left(C_{A / k, Q}, d\right)$ for the derivation system. Note in particular that the construction, for $Q:=\Omega_{A / k}^{1}$, yields the non-commutative version of Iitaka's differentials mentioned at the end of (5.6).

The affine analogue of the construction given by Demailly [8] is obtained from the composite map $\Omega_{A}^{1} \otimes_{A} \tilde{A} \rightarrow \tilde{A}$ of $\kappa \otimes_{A} 1$ with the natural map $Q \otimes_{A} \tilde{A} \rightarrow \tilde{A}$. We obtain a diagram analogous to (5.9.1), which defines $\tilde{Q}$ :

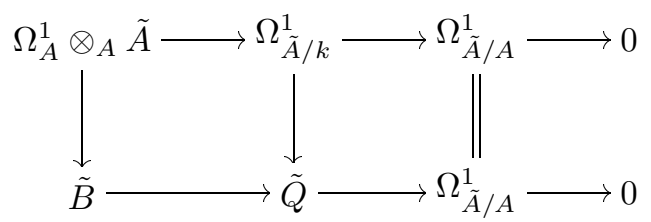

with exact rows, and where $\tilde{B}$ is the image of $Q \otimes_{A} \tilde{A} \rightarrow \tilde{A}$. The bottom sequence is analogous to the sequence (4.2) of Demailly [8]. If we let $\tilde{X}=\operatorname{Proj}\left(\operatorname{Sym}_{A}(Q)\right)$, we obtain a surjective map $Q \otimes_{\mathcal{O}_{X}} \mathcal{O}_{\tilde{X}} \rightarrow \mathcal{O}_{X}(1)$ and the bottom line gives the exact sequence

$$
0 \rightarrow \mathcal{O}_{X}(1) \rightarrow \tilde{Q} \rightarrow \Omega_{\tilde{X} / X}^{1} \rightarrow 0,
$$

which is the dual of the sequence of Demailly.

5.10. Example (Meyer). Let $X$ be an $m$-dimensional $\mathcal{C}^{\infty}$-manifold. Let $A:=$ $\mathcal{C}^{\infty}(X)$ be the $k$-algebra of global $k$-valued functions, where $k=\mathbf{R}$ or $k=\mathbf{C}$, and let $\Omega^{1}(X)$ be the $A$-module of global 1-forms.

Let $T X$ be the tangent bundle over $X$, and let $\tilde{A}:=\mathcal{C}^{\infty}(T X)$. Then there are an inclusion of algebras $\iota: A \rightarrow \tilde{A}$ induced by the projection $T X \rightarrow X$, and an inclusion of $A$-modules $\Omega^{1}(X) \rightarrow \mathcal{C}^{\infty}(T X)$. In particular, the differential may be viewed as a $k$-linear module derivation $d: A \rightarrow \tilde{A}$. In addition, the projection $T X \rightarrow X$ induces an $A$-linear map $i: \Omega^{1}(X) \rightarrow \Omega^{1}(T X)$ and the following diagram is commutative:

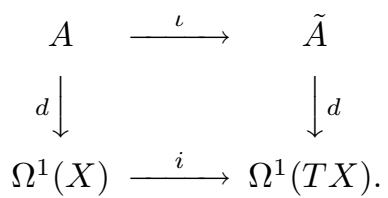

In addition, on $T X$ there is a canonical tangent vector field $\Xi$, defined at a point $(x, \xi)$ of $T X$ as the directional derivative along the line from $(x, 0)$ to $(x, \xi)$. Hence, evaluation of 1-forms of $T X$ on the field $\Xi$ defines a canonical map $\tilde{A}$-linear map

$$
r: \Omega^{1}(T X) \rightarrow \tilde{A} \text {. }
$$

Moreover, if $\omega \in \Omega^{1}(X)$, then $r d \omega=\omega$.

In local coordinates, say if $X$ is an open subset of $\mathbf{R}^{m}$ with coordinate functions $x_{j}$, we have that the $2 m$ functions $\iota x_{j}$ and $d x_{j}$ are coordinate functions on $T X$. The $A$-module $\Omega^{1}(X)$ is free with basis $d x_{j}$, and the $\tilde{A}$-module $\Omega^{1}(T X)$ is free with basis $d \iota x_{j}$ and $d d x_{j}$. Under the map $i$, the differential $d x_{j}$ is mapped to $d \iota x_{j}$. Under the map $r$, the differential $d d x_{j}$ is mapped to $d x_{j}$ and $d \iota x_{j}$ is mapped to 0 .

The derivation system of Meyer [22] is defined as follows: Let $C_{0}:=A$ and, inductively, let $C_{n}:=\mathcal{C}^{\infty}\left(T^{n} X\right)$ be the $k$-algebra of $\mathcal{C}^{\infty}$-functions on the iterated tangent space $T^{n} X$. Then $C_{n+1}=\tilde{C}_{n}$ and we have obtained a derivation system 
$\left(C_{X}, d\right)$ with the additional structure of (5.6). Note that $\Omega^{n+1}(C) \subseteq \Omega^{1}\left(T^{n} X\right)$. If $X$ is paracompact, then $\Omega^{1}(C)=\Omega^{1}(X)$.

The system is a Kähler system, since compositions of the maps $i$ of (5.10.1) may be used as the $A$-linear maps $i_{n}$ of (5.6).

\section{KÄHLER BASES}

We say that a Kähler system $(C, d)$ has a Kähler basis if there are elements $x_{1}, \ldots, x_{m}$ of $A=C_{0}$ such that $d x_{1}, \ldots, d x_{m}$ is an $A$-basis for $\Omega^{1}(C)$, and such that the subset $\mathcal{X}_{n}$ of $C_{n}$, for $n=1,2, \ldots$, defined inductively by $\mathcal{X}_{1}=\left\{d x_{1}, \ldots, d x_{m}\right\}$ and

$$
\mathcal{X}_{n+1}=\left\{d \xi \mid \xi \in X_{n}\right\} \cup\left\{d x_{j} . \xi \mid j=1, \ldots, m, \xi \in \mathcal{X}_{n}\right\},
$$

form an $A$-basis for the $n$ 'th graded piece $\Omega^{n}(C)$ of $\Omega(C)$.

We show for a Kähler system with a Kähler basis that for each integer $n \geq 1$ there is an exact sequence of $A$ - $A$-modules

$$
0 \rightarrow \Omega^{1}(C) \otimes_{A} \Omega^{n}(C) \rightarrow \Omega^{n+1}(C) \rightarrow \Omega^{n}(C) \rightarrow 0
$$

which is split by right $A$-linear maps.

The main result of this section, and indeed the article, is that a Kähler system with a Kähler basis satisfies the criteria of the main result of Section 4. In particular we obtain, for any $\varepsilon$ in $k$, that $\Omega(C)$ is a $k$-algebra over $A$ and $C$ is a natural $\Omega(C)$ module. We also obtain an explicit formula for the action of $\Omega(C)$ on $C$.

It is an immediate consequence of our results that both the system of higher order differentials for $C^{\infty}$-manifolds constructed by P.-A. Meyer, and our noncommutative generalization of Iitaka's higher order differentials for smooth schemes give rise to Kähler systems with Kähler bases. In particular, the higher order differentials of both these systems have multiplicative structures.

In a final note we indicate how the theory can be globalized.

6.1. Setup. Let $(C, d)$ be a Kähler system. For a given subset $\mathcal{X}=\left\{x_{1}, \ldots, x_{m}\right\}$ of $A$, define a sequence $\mathcal{X}_{n}$ of subsets of $C_{n}$ for $n=1,2, \ldots$ as follows: The set $\mathcal{X}_{1}$ consists of the 1 -forms $d x_{j}$ and, inductively, the set $\mathcal{X}_{n+1}$ consists of the elements of one of the following two forms:

$$
\xi_{0}:=d \xi, \quad \xi_{j}:=d x_{j} . \xi \quad \text { for } \xi \in \mathcal{X}_{n} .
$$

By (5.2.1) we have that $\xi_{j}=d\left(x_{j} \xi\right)-x_{j} d \xi$. In particular, it follows by induction that $\mathcal{X}_{n}$ is a subset of $\Omega^{n}(C)$. Moreover, if the elements $d x_{j}$ generate $\Omega^{1}(C)$ as an $A$-module, then the elements of $\mathcal{X}_{n}$ generate $\Omega^{n}(C)$ as an $A$-module. Indeed, assume that the elements $d x_{j}$ generate $\Omega^{1}(C)$. The elements of $\Omega^{n+1}(C)$ are $A$ linear combinations of elements of the form $d \omega$ for $\omega \in \Omega^{n}(C)$. So, if the elements $\xi$ of $\mathcal{X}_{n}$ generate $\Omega^{n}(C)$, then the elements of $\Omega^{n+1}(C)$ are $A$-linear combinations of elements of the form

$$
d(f \xi)=d f \cdot \xi+f d \xi .
$$

Since $d f$ is an $A$-linear combination of the $d x_{j}$, an element of the form (6.1.2) is an $A$-linear combination of elements of the form (6.1.1). Therefore, by induction, the elements of $\mathcal{X}_{n+1}$ generate $\Omega^{n+1}(C)$.

6.2. Lemma. Assume in the setup of (6.1) that the elements $d x_{j}$ form an A-basis of $\Omega^{1}(C)$. Then the following three conditions are equivalent: 
(i) For all $n \geq 1$, if $\omega_{0}, \omega_{1}, \ldots, \omega_{m}$ are elements of $\Omega^{n}(C)$ such that

$$
d \omega_{0}+\sum_{j=1}^{m} d x_{j} \cdot \omega_{j}=0
$$

then $\omega_{0}=\omega_{1}=\cdots=\omega_{m}=0$.

(ii) For all $n \geq 1$, the elements of $\mathcal{X}_{n}$ are linearly independent over $A$.

(iii) For all $n \geq 1$, there are two k-linear maps, $r: \Omega^{n+1}(C) \rightarrow \Omega^{n}(C)$ and $s: \Omega^{n+1}(C) \rightarrow \Omega^{1}(C) \otimes_{A} \Omega^{n}(C)$, such that, for $f \in A$ and $\omega \in \Omega^{n}(C)$,

$$
r(f d \omega)=f \omega, \quad s(f d \omega)=-d f \otimes_{A} \omega .
$$

Proof. We will give a cyclic proof of the equivalence. Assume first (i). The elements of $\mathcal{X}_{1}$ are linearly independent by hypothesis. Proceed by induction and consider a linear relation among the elements of $\mathcal{X}_{n+1}$. By the inductive definition of $\mathcal{X}_{n+1}$, the relation is of the form

$$
\sum_{i} g_{i} d \xi_{i}+\sum_{j=1}^{m} \sum_{i} f_{i j} d x_{j} \cdot \xi_{i}=0,
$$

where the $\xi_{i}$ are different elements of $\mathcal{X}_{n}$ and $g_{i}$ and $f_{i j}$ belong to $A$. Since $d g . \xi=$ $d(g \xi)-g d \xi$, the relation may be written as follows:

$$
d\left(\sum_{i} g_{i} \xi_{i}\right)-\sum_{i} d g_{i} \cdot \xi_{i}+\sum_{j=1}^{m} d x_{j} \cdot \sum_{i} f_{i j} \xi_{i}=0 .
$$

Since $d g$, for any $g \in A$, is an $A$-linear combination of the $d x_{j}$, it follows that the middle sum is a sum of terms of the form $d x_{j} \cdot \omega_{j}$. Hence it follows from (i) and (6.2.3) that $\sum g_{i} \xi_{i}=0$. Hence, since the $\xi_{i}$ are linearly independent by the induction hypothesis, we have that $g_{i}=0$ for all $i$. So the first two sums in (6.2.3) vanish. Hence it follows from (i) and (6.2.3) that $\sum_{i} f_{i j} \xi_{i}=0$ for $j=1, \ldots, m$. Again, since the $\xi_{i}$ are independent, it follows that $f_{i j}=0$ for all $i$ and $j$. Hence (ii) holds.

Assume next (ii). Since the $d x_{j}$ generate $\Omega^{1}(C)$, it follows, as noted on (6.1), that the elements of $\mathcal{X}_{n}$ generate $\Omega^{n}(C)$. So, since (ii) is assumed, the elements of $\mathcal{X}_{n+1}$ form an $A$-basis of $\Omega^{n+1}(C)$. Therefore, there are well defined maps $r$ : $\Omega^{n+1}(C) \rightarrow \Omega^{n}(C)$ and $s: \Omega^{n+1}(C) \rightarrow \Omega^{1}(C) \otimes_{A} \Omega^{n}(C)$ determined additively, for $\xi \in \mathcal{X}_{n}$ and $f \in A$, by the equations

$$
\begin{aligned}
r(f d \xi)=\xi, & r\left(f d x_{j} \cdot \xi\right)=0, \\
s(f d \xi)=-d f \otimes_{A} \xi, & s\left(f d x_{j} \cdot \xi\right)=f d x_{j} \otimes_{A} \xi .
\end{aligned}
$$

In the equations (6.2.2), the element $\omega$ is an $A$-linear combination of elements $\xi$ in $\mathcal{X}_{n}$. So, to verify (6.2.2), we may assume that $\omega=g \xi$ with $g \in A$ and $\xi \in \mathcal{X}_{n}$. Then $f d \omega=f d g . \xi+f g d \xi$. The element $d g$ is an $A$-linear combination of the $d x_{j}$. Hence, from the equations (6.2.4) it follows that

$$
r(f d \omega)=r(f d g . \xi)+r(f g d \xi)=f g \xi=f \omega .
$$

From the last equation in (6.2.5) it follows by additivity that $s(f d g . \xi)=f d g \otimes_{A} \xi$. Consequently, from the first equation in (6.2.5), it follows that

$$
s(f d \omega)=f d g \otimes_{A} \xi-d(f g) \otimes_{A} \xi=-g d f \otimes_{A} \xi=-d f \otimes_{A} g \xi=-d f \otimes_{A} \omega .
$$

Therefore, the two equations of (6.2.2) have been verified. Hence (iii) holds. 
Assume finally (iii). Since $d(f \omega)=d f . \omega+f d \omega$, it follows easily from (6.2.2) that $r(d f . \omega)=0$ and $s(d f . \omega)=d f \otimes_{A} \omega$ for $f \in A$ and $\omega \in \Omega^{n}(C)$. Hence, when we apply $r$ to the relation (6.2.1) we obtain the equation $\omega_{0}=0$ and when we apply $s$ we obtain the equation $\sum_{j} d x_{j} \otimes_{A} \omega_{j}=0$ in $\Omega^{1}(C) \otimes_{A} \Omega^{n}(C)$. Since the $d x_{j}$ form an $A$-basis for $\Omega^{1}(C)$, it follows that $\omega_{1}=\cdots=\omega_{m}=0$. Hence (i) holds.

Thus the lemma has been proved.

6.3. Definition. A subset $\mathcal{X}=\left\{x_{1}, \ldots, x_{m}\right\}$ of $A$ such that the 1 -forms $d x_{j}$ form an $A$-basis for $\Omega^{1}(C)$ and such that the equivalent conditions of Lemma (6.2) hold will be called a Kähler basis for the Kähler system $(C, d)$.

For instance, for the system $\left(\Omega_{A / k}, d_{A / k}\right)$ of free Kähler differentials of Example (5.5), the maps $r:=r_{A / k}$ and $s:=s_{A / k}$ have the properties of (6.2)(iii). Thus any subset $\left\{x_{1}, \ldots, x_{m}\right\}$ of $A$ such that the differentials $d x_{j}$ form an $A$-basis for $\Omega_{A / k}^{1}$ is a Kähler basis for $\left(\Omega_{A / k}, d_{A / k}\right)$, as we also observed in (3.12).

6.4. Note. Clearly, for a given Kähler system $(C, d)$, there is at most one pair of maps $r$ and $s$ with the properties in (6.2.2). Assume that $r=r_{C}$ and $s=s_{C}$ exist. Denote by $i_{C}: \Omega^{1}(C) \otimes_{A} \Omega^{n}(C) \rightarrow \Omega^{n+1}(C)$ the $A$-linear map induced by the multiplication of (5.2). Then there is a commutative diagram of $A$-linear maps,

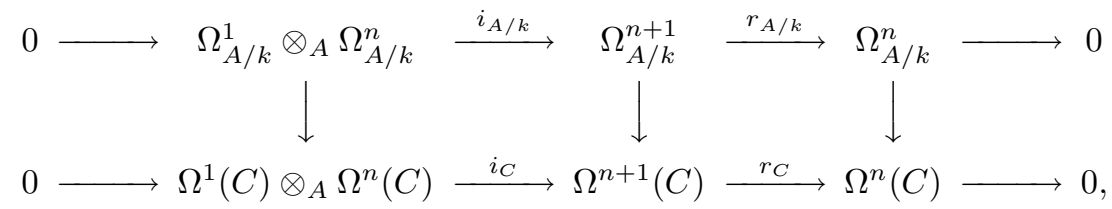

where the vertical maps are the surjections induced by the maps (6.1.7). Indeed, the first square of the diagram commutes because of (5.2.2), and the second square commutes because the maps are $A$-linear and $r_{C} d=1$ and $r_{A / k} d_{A / k}=1$.

The top row of the diagram is split exact by (3.9). The bottom row is exact, and split by the $k$-linear maps $s_{C}$ and $d$. To prove the latter assertion, note that, for $\rho \in \Omega^{1}(C)$ and $\pi \in \Omega^{n}(C)$, we have the equations

$$
r(\rho . \pi)=0, \quad s(\rho . \pi)=\rho \otimes_{A} \pi .
$$

Indeed, in (6.4.1) we may assume that $\rho=a d b$. Then $\rho . \pi=a d(b \pi)-a b d \pi$. Hence it follows from the two equations of (6.2.2) that $r(\rho . \pi)=a b \pi-a b \pi=0$ and $s(\rho . \pi)=-d a \otimes_{A} b \pi+d(a b) \otimes_{A} \pi=a d b \otimes_{A} \pi=\rho \otimes_{A} \pi$. Now, given the equations (6.2.2) and (4.4.1) the exactness property is proved exactly as in the proof of (2.7).

Note, in particular, that if $\Omega_{A / k}^{1}=\Omega^{1}(C)$, then if follows from the properties of the diagram that $\Omega_{A / k}^{n}=\Omega^{n}(C)$ for all $n$.

6.5. Lemma. Assume that $(C, d)$ is a Kähler system. Let $\mathcal{X}=\left\{x_{1}, \ldots, x_{m}\right\}$ be a subset of $A$ such that the 1-forms $d x_{j}$ form an $A$-basis for $\Omega^{1}(C)$. For any polynomial $Q$ in $\Lambda_{A / k}^{\varepsilon, n+1}$, define polynomials $Q_{j}$ in $\Lambda_{A / k}^{\varepsilon, n}$ for $j=1, \ldots, m$ by the following expansion in $\Omega^{1}(C) \otimes_{A} \Lambda_{A / k}^{\varepsilon, n}$ :

$$
s_{C, t}^{\varepsilon}(Q)=\sum_{j=1}^{m} d x_{j} \otimes_{A} Q_{j}
$$


where $s_{C, t}^{\varepsilon}$ is the map of (6.1.5). In addition, let $Q_{0}:=r_{t}^{\varepsilon} Q$ where $r_{t}^{\varepsilon}$ is the map of (2.6). Then, for any element $F \in C$, we have the equation

$$
Q(d)(F)=d Q_{0}(d)(F)-\varepsilon^{n} Q_{0}(d)(d F)+\sum_{j=1}^{m} d x_{j} \cdot Q_{j}(d)(F) .
$$

Proof. Set $H:=\Lambda_{A / k}^{1} \otimes_{A} \Lambda_{A / k}^{\varepsilon, n}$. The $A$-linear map $i_{t}^{\varepsilon}: H \rightarrow \Lambda_{A / k}^{\varepsilon, n+1}$ of (2.4.1) is induced by the multiplication in the algebra $\Lambda_{A / k}^{\varepsilon}$ and it maps $H$ isomorphically onto its image. So we may view $H$ as an $A$-submodule of $\Lambda_{A / k}^{\varepsilon, n+1}$. If $n=0$, then $H=\Lambda_{A / k}^{1}$. If $n>0$, then, by Proposition (2.7), the $A$-submodule $H$ has the $k$ submodule $d_{t}^{\varepsilon}\left(\Lambda_{A / k}^{\varepsilon, n}\right)$ as complement. Therefore, by additivity, it suffices to prove (6.5.1) separately for the case when $Q$ belongs to $H$ and for the case when $n>0$ and $Q$ is of the form $d_{t}^{\varepsilon} R$ for $R \in \Lambda_{A / k}^{\varepsilon, n}$.

Consider the first case. It follows from the second equation of (6.1.4) that the map $s_{C, t}^{\varepsilon}$ is $A$-linear on the submodule $H$. Moreover, if $Q$ belongs $H$, then, again by Proposition (2.7), we have that $Q_{0}=0$. It follows that the two sides of (6.5.1) are $A$-linear as functions of $Q$ in $H$. Hence, to prove the formula in the first case, we may assume that $Q=\left(\Delta_{t} f\right) R$ for $f \in A$ and $R \in \Lambda_{A / k}^{\varepsilon, n}$. Let $d f=\sum_{j=1}^{m} f_{j} d x_{j}$ be the expansion of $d f$. Since $Q=\left(\Delta_{t} f\right) R=t f R-f t R$, we obtain by (5.2.1) the following expression for the left side of (6.5.1):

$$
d(f R(d)(F))-f d R(d)(F)=d f \cdot R(d)(F)=\sum_{j=1}^{m} f_{j} d x_{j} \cdot R(d)(F) .
$$

Since $Q=\left(\Delta_{t} f\right) R$, it follows from the second equation of (6.1.4) that $s_{C, t}^{\varepsilon}(Q)=$ $d f \otimes_{A} R$. Hence $Q_{j}=f_{j} R$ for $j=1, \ldots, m$. Moreover, $Q_{0}=0$. Hence we have the following expression for the right side of (6.5.1):

$$
\sum_{j=1}^{m} d x_{j} \cdot f_{j} R(d)(F) .
$$

Clearly, the two expressions (6.5.2) and (6.5.3) are equal. Thus (6.5.1) has been proved in the first case.

Consider the second case. Then $Q=d_{t}^{\varepsilon} R$ where $R$ is of degree $n$ and $n \geq 1$. It follows from the first equation of (6.1.4) that $s_{C, t}^{\varepsilon}(Q)=0$. Hence $Q_{j}=0$ for $j=1, \ldots, m$ and the sum in (6.5.1) vanishes. Moreover, since $n \geq 1$, we have that $Q_{0}=r_{t}^{\varepsilon} d_{t}^{\varepsilon} R=R$. Hence $Q=d_{t}^{\varepsilon} Q_{0}=t Q_{0}-\varepsilon^{n} Q_{0} t$, and so (6.5.1) holds.

Thus Equation (6.5.1) has been proved in both cases.

6.6. Lemma. Assume that the Kähler system $(C, d)$ has a Kähler basis. Then, with $\varphi:=d$ and $u:=1$, the equivalent conditions of Proposition (4.4) are satisfied.

Proof. Let $\mathcal{X}$ be a Kähler basis for the system. We verify the condition (ii) of (4.4), that is, if $Q$ is a polynomial in $\Lambda_{A / k}^{\varepsilon}$ such that $Q(d)(1)=0$, then $Q(d)=0$. Since the endomorphism $d$ is homogeneous of degree 1 , it suffices to verify the condition for homogeneous polynomials $Q$.

We prove, by induction on $n$, that if $Q \in \Lambda_{A / k}^{\varepsilon, n}$ and $Q(d)(1)=0$, then $Q(d)=0$.

If $n=0$, then $Q$ is an element $f$ of $A$, and $Q(d)$ left multiplication by $f$. Hence, if $Q(d)(1)=0$, it follows that $f=0$ and hence that $Q(d)=0$. 
In general, assume that $Q \in \Lambda_{A / k}^{\varepsilon, n+1}$ where $n \geq 0$. Then, by Lemma (6.5), there are polynomials $Q_{0}$ and $Q_{j}$ in $\Lambda_{A / k}^{\varepsilon, n}$ such that, for all $F \in C$, the following equation holds:

$$
Q(d)(F)=d Q_{0}(d)(F)-\varepsilon^{n} Q_{0}(d)(d F)+\sum_{j=1}^{m} d x_{j} \cdot Q_{j}(d)(F) .
$$

For $F=1 \in C_{0}$, the equation reduces to the following:

$$
Q(d)(1)=d Q_{0}(d)(1)+\sum_{j=1}^{m} d x_{j} \cdot Q_{j}(d)(1) .
$$

Assume that $Q(d)(1)=0$. Then, by Condition (6.2)(i) applied with $\omega:=Q_{0}(d)(1)$ and $\omega_{j}:=Q_{j}(d)(1)$, it follows from (6.6.2) that $Q_{0}(d)(1)=Q_{1}(d)(1)=\cdots=$ $Q_{m}(d)(1)=0$. By the induction hypothesis, the latter equations imply that $Q_{0}(d)=Q_{1}(d)=\cdots=Q_{m}(d)=0$. It follows that the right side of (6.6.1) is equal to 0 for any $F \in C$. Hence $Q(d)=0$.

Thus the condition (ii) of Proposition (4.4) is satisfied.

6.7. Theorem. Assume that the Kähler system $(C, d)$ has a Kähler basis. Then the following assertions hold:

(1) For every $n \geq 1$, there are an A-linear map $r: \Omega^{n+1}(C) \rightarrow \Omega^{n}(C)$ and a k-linear map $s: \Omega^{n+1}(C) \rightarrow \Omega^{1}(C) \otimes_{A} \Omega^{n}(C)$, determined, for $f \in A$ and $\omega \in \Omega^{n}(C)$, by the equations

$$
r(f d \omega)=f \omega, \quad s(f d \omega)=-d f \otimes_{A} \omega .
$$

Moreover, the following sequence of A-linear maps is exact:

$$
0 \rightarrow \Omega^{1}(C) \otimes_{A} \Omega^{n}(C) \stackrel{i}{\rightarrow} \Omega^{n+1}(C) \stackrel{r}{\rightarrow} \Omega^{n}(C) \rightarrow 0,
$$

and split by the $k$-linear maps $d$ and s. Finally, the diagram of (6.4) is commutative.

(2) For any $\varepsilon \in k$, there is a unique A-linear product,

$$
\Omega(C) \otimes_{k} C \rightarrow C,
$$

denoted $\omega \otimes F \mapsto \omega . F$, such that $1 \in A$ is a left unit and such that the endomorphism $d$ is an $\varepsilon$-derivation with respect to the product, that is, such that

$$
d(\omega \cdot F)=(d \omega) \cdot F+\left(\varepsilon_{\mathrm{gr}} \omega\right) \cdot d F
$$

for $\omega \in \Omega(C)$ and $F \in C$. The product is given, for $P \in A\langle t\rangle$ and $F \in C$, by the formula

$$
P(d)(1) \cdot F=\sum_{i=0}^{\infty}(-1)^{i} \varepsilon^{i(i-1) / 2} \partial_{i}^{\varepsilon} P(d) d^{i} F
$$

The A-submodule $\Omega(C)$ of $C$ is stable under the product and, with the induced product, $\Omega(C)$ is a graded $k$-algebra over $A$ and $C$ is a graded $\Omega(C)$-module.

Moreover, the canonical map $\Omega_{A / k} \rightarrow \Omega(C)$ of (6.1.7) is a surjection of graded $k$-algebras over $A$,

$$
\Omega_{A / k}^{\varepsilon} \rightarrow \Omega(C),
$$

and it is an isomorphism when the surjection $\Omega_{A / k}^{1} \rightarrow \Omega^{1}(C)$ is an isomorphism.

Finally, the product in degree 1 , that is, $\rho . F$ for $\rho \in \Omega^{1}(C)$ and $F \in C$, is equal to the product of (5.2). 
Proof. (1) The existence of the maps $r$ and $s$ is the condition (iii) of (6.2). Hence the existence follows from the existence of a Kähler basis. As noted in (6.4), the remaining properties in (1) are consequences of (6.7.1).

(2) The existence of the product with the required property, the formula (6.7.2) and the fact that $\Omega(C)$ is a $k$-algebra over $A$ and $C$ an $\Omega(C)$-module follow from Proposition (4.4). In addition, since the map $\Lambda_{A / k}^{\varepsilon} \rightarrow \Omega(C)$ is a map of $k$-algebras over $A$ and since, as we saw in (6.1), it factors over the quotient $\Omega_{A / k}^{\varepsilon}$, it induces a map of algebras from the quotient. As noted in (6.4), the map is an isomorphism if it is an isomorphism in degree 1.

To prove the final assertion, it suffices to prove that the equation $d(f F)=$ $d f . F+f d F$ holds for both products. It holds for the product in (6.1) by definition, and it holds for the product defined by (6.7.2), since $d$ is an $\varepsilon$-derivation and $f$ is of degree 0.

Thus all the assertions of the theorem have been proved.

6.8. Note. Assume that $\mathcal{X}$ is a given Kähler basis for the derivation system $(C, d)$. Since the elements of $\mathcal{X}_{n}$ form an $A$-basis for $\Omega^{n}(C)$, the $\varepsilon$-product $\omega . F$, for $\omega \in$ $\Omega^{n}(C)$ and $F \in C_{p}$, is completely determined by the products $\xi . F$ for $\xi \in \mathcal{X}_{n}$. The latter products are determined inductively as follows:

First, for $n=1$ and any $f \in A$, we have that

$$
d f . F=d(f F)-f d F .
$$

In particular, the latter equation determines the products $d x_{j} . F$.

Inductively, the elements of $\mathcal{X}_{n+1}$ are the elements $\xi_{0}=d \xi$ and $\xi_{j}:=d x_{j} . \xi$ for $\xi \in \mathcal{X}_{n}$. Clearly,

$$
\xi_{0} \cdot F=d \xi . F=d(\xi . F)-\xi . d F,
$$

where the terms on the right side are determined inductively. Similarly,

$$
\xi_{j} \cdot F=d x_{j} \cdot \xi \cdot F=d\left(x_{j} \xi \cdot F\right)-x_{j} d(\xi . F)
$$

is determined inductively. This is the method used by Meyer [22] to define the multiplication of higher order differentials on manifolds. The task of showing that the multiplication is well defined and associative is formidable.

Note that the number of elements in the basis $\mathcal{X}_{n}$ is equal to $(m+1)^{n-1} m$. It follows, exactly as in the proof of (3.13), that the set of all $\varepsilon$-products $d^{n_{1}} x_{j_{1}} \ldots d^{n_{l}} x_{j_{l}}$, for $n_{1}+\cdots+n_{l}=n$ and each $n_{i}>0$, form an $A$-basis for $\Omega^{n}(C)$. Moreover, in the latter basis, the endomorphism $d$ and the $\varepsilon$-multiplication are determined as in (3.14).

6.9. Example. Consider the Kähler system $\left(C_{A / k, Q}, d\right)$ of Example (5.9), defined by a quotient $\Omega_{A / k}^{1} \rightarrow Q$. Thus, for $x \in A$, the differential $d x$ is the image of $d_{A / k} x$ in $Q$. Assume that $Q$ is a free $A$-module with a basis formed by differentials $d x_{j}$ for some subset $\mathcal{X}=\left\{x_{1}, \ldots, x_{m}\right\}$ of $A$. Then the assertions (1) and (2) of Theorem (6.7) hold.

Indeed, it suffices to prove that $\mathcal{X}$ is a Kähler basis for the system. We verify the condition (i) of (6.2).

In the notation of (5.9), it follows by induction on $n$ from the split exact sequence in the diagram (5.9.2) that $Q_{n+1}$ is a free $C_{n}$-module and that the elements $i_{n} d x_{j}=$ $d \iota^{n} x_{j}$ of $Q_{n}$ form a subset of a $C_{n}$-basis. To verify the condition (i), consider a 
relation (6.2.1). Since $d x_{j} . F=\left(d \iota^{n} x_{j}\right)(\iota F)$ by (5.6.1), the relation may be written as follows:

$$
d \omega_{0}+\sum_{j=1}^{m}\left(\iota \omega_{j}\right) d \iota^{n} x_{j}=0
$$

Apply the map $r$ of (5.9.2). The map $r$ is $C_{n}$-linear and $r i=0$. Since $d \iota^{n}=i_{n} d$, it follows that the sum in (6.9.1) is mapped to 0. Moreover $r d \omega=\omega$ for $\omega \in Q_{n}$. Thus it follows from (6.9.1) that $\omega_{0}=0$. Hence the sum in (6.9.1) vanishes. As the $d \iota^{n} x_{j}$ form a subset of a $C_{n}$-basis, it follows that $\omega_{1}=\cdots=\omega_{m}=0$. Thus the condition (i) has been verified.

Note in particular that if $\Omega_{A / k}^{1}$ is a free $A$-module with a basis of the form $d x_{j}$, then the two assertions (1) and (2) of Theorem (6.7) hold for the non-commutative version of Iitaka's differentials.

6.10. Example. Consider similarly the system $\left(C_{X}, d\right)$ of Meyer of Example (5.10) defined from an $m$-dimensional $\mathcal{C}^{\infty}$-manifold $X$. Assume that $X$ is an open subset of $\mathbf{R}^{m}$. Then the assertions (1) and (2) of Theorem (6.7) hold.

Indeed, it suffices to prove that set $\mathcal{X}=\left\{x_{1}, \ldots, x_{m}\right\}$ of coordinate functions is a Kähler basis for the system. The proof is identical to the proof given for the system in (6.9), using the maps $i$ and $r$ of (5.10).

6.11. Note. For $f \in A, \pi \in \Omega^{p}(C)$ and $F \in C$, we have the formula $f d \pi . F=$ $d(f \pi F)-\varepsilon^{p} f \pi . d F$. Using this formula, it is easy to deduce from the two equations of (6.7.1) the following formulas:

$$
r(\omega \cdot F)=r(\omega) \cdot F-\varepsilon^{p} r(r(\omega) \cdot d F), \quad s(\omega \cdot F)=s(\omega) \cdot F-\varepsilon^{p} s(r(\omega) \cdot d F),
$$

for $\omega \in \Omega^{p+1}(C)$ and $F \in \Omega(C)$. The iterated version of the formula for $r$ was given, for manifolds and for $\varepsilon=1$, by Meyer [22, p. 261].

6.12. Globalization. Clearly, the constructions of this section are functorial in the following sense: Given a homomorphism $k \rightarrow k^{\prime}$ of commutative rings and a commutative $k^{\prime}$-algebra $A^{\prime}$. View $A^{\prime}$ as a $k$-algebra and let $\varphi: A \rightarrow A^{\prime}$ be a homomorphism of $k$-algebras. Then there are induced homomorphisms

$$
\left(\Lambda_{A / k}, d_{A / k}, r_{A / k}, s_{A / k}\right) \rightarrow\left(\Lambda_{A^{\prime} / k^{\prime}}, d_{A^{\prime} / k^{\prime}}, r_{A^{\prime} / k^{\prime}}^{\prime}, s_{A^{\prime} / k^{\prime}}\right),
$$

and similar homomorphisms of free Kähler differentials. It follows that the constructions globalize as follows: Fix a topological space $X$. Let $k$ be a presheaf of commutative rings on $X$. In addition, let $\mathcal{A}$ be a sheaf of $k$-algebras on $X$. By the functoriality, the assignments, for open subsets $U$ of $X$, of

$$
\Lambda_{\mathcal{A}(U) / k(U)} \text { and } \Omega_{\mathcal{A}(U) / k(U)}
$$

are presheaves on $X$. Denote by

$$
\Lambda_{\mathcal{A} / k} \text { and } \Omega_{\mathcal{A} / k}
$$

the associated sheaves. Clearly, the sheaves are sheaves of $\mathcal{A}$-modules, and the maps $d, r, s, i$ induce maps of sheaves.

For instance, on a differentiable manifold $X$, we obtain from the system of Meyer a Kähler system of sheaves $\mathcal{C}_{X}$ with $\mathcal{C}_{X, n}:=p_{n *} \mathcal{C}_{T^{n} X}^{\infty}$ (where $p_{n}: T^{n} X \rightarrow X$ is the projection) and for every $n$ a subsheaf $\Omega_{X}^{n} \subseteq \mathcal{C}_{X, n}$. From Theorem (6.7) we 
obtain, for every $\varepsilon \in k$, a $\mathcal{C}_{X}^{\infty}$-linear map of sheaves,

$$
\Omega_{X}^{p} \otimes \mathcal{C}_{X, n} \rightarrow \mathcal{C}_{X, n+p}
$$

satisfying the assertions of (6.7) on sections.

Assume that a map of schemes $p: X \rightarrow S$ is given. Then, from the algebra $\mathcal{A}:=\mathcal{O}_{X}$ as a sheaf of $p^{-1} \mathcal{O}_{S}$-algebras, we obtain quasi coherent $\mathcal{O}_{X}$-modules $\Lambda_{X / S}^{n}$ and $\Omega_{X / S}^{n}$.

If $\mathcal{Q}$ is a given quasi-coherent quotient of $\Omega_{X / S}^{1}$, we obtain, from the system Example (5.9), a Kähler system $\left(\mathcal{C}_{X / S, \mathcal{Q}}, d\right)$. If $\mathcal{Q}$ is locally free, then the system has locally a Kähler basis. It follows that the product defined in Theorem (6.7) exists globally.

In particular, if $S=\operatorname{Spec} k$ and $X=\operatorname{Spec} A$ are affine, and $\mathcal{Q}=\tilde{Q}$ with $Q$ a locally free $A$-module, then the two assertions of Theorem (6.7) hold.

If $X$ is smooth over $S$, we may take $\mathcal{Q}=\Omega_{X / S}^{1}$ to obtain a Kähler system $\left(\mathcal{C}_{X / S}, d\right)$ of quasi-coherent $\mathcal{O}_{X}$-modules and locally free submodules $\Omega_{X / S}^{n} \subseteq \mathcal{C}_{X / S}^{n}$. Moreover, for every global section $\varepsilon$ on $S$, we have that $\Omega_{X / S}$ is a graded (noncommutative) $\mathcal{O}_{X}$-algebra, and $\mathcal{C}_{X / S}$ is a module over $\Omega_{X / S}$ and $d$ is an $\varepsilon$-derivation.

\section{Symmetric AND ALternating DifFERENTIALS}

In this section we assume that $\varepsilon^{2}=1$. We show that the $k$-linear endomorphism of $\Lambda_{A / k}^{\varepsilon}$ of degree -1 described by

$$
\delta_{t}^{\varepsilon}\left(a_{n} t \cdots t a_{0}\right)=\sum_{i=1}^{n-1} i \varepsilon^{n-i-1} a_{n} t \cdots t a_{i+1} a_{i} t \cdots t a_{0}
$$

is an $\varepsilon$-derivation on $\Lambda_{A / k}^{\varepsilon}$, and that it induces an $\varepsilon$-derivation $\delta_{t}$ on the Kähler differentials $\Omega_{A / k}^{\varepsilon}$. We show that the left ideal of $\Lambda_{A / k}^{\varepsilon}$ generated by the $\varepsilon$-commutators $[P, Q]_{\varepsilon}:=P Q-\varepsilon^{\operatorname{deg} P \operatorname{deg} Q} Q P$, when $P$ and $Q$ are homogeneous, is a two sided ideal $\mathfrak{C}_{\varepsilon}$. The quotient of $\Lambda_{A / k}^{\varepsilon}$ by $\mathfrak{C}_{\varepsilon}$ is the algebra $\Omega_{A / k}^{\varepsilon \text {-sym }}$ of $\varepsilon$-symmetric differentials. The $\varepsilon$-derivations $d_{t}^{\varepsilon}$ and $\delta_{t}^{\varepsilon}$ of $\Lambda_{A / k}^{\varepsilon}$ induce $\varepsilon$-derivations on $\Omega_{A / k}^{\varepsilon \text {-sym }}$. When $\varepsilon=1$, we obtain the algebra $\Omega_{A / k}^{\mathrm{sym}}$ of symmetric differentials and for $\varepsilon=-1$ we obtain the algebra $\Omega_{A / k}^{\text {skew }}$ of skew symmetric differentials. The algebra obtained from $\Omega_{A / k}^{\text {skew }}$ by division by the ideal generated by all squares of elements of odd degree is the algebra $\Omega_{A / k}^{\text {alt }}$ of alternating differentials. All these algebras are graded and $d_{t}^{\varepsilon}$ and $\delta_{t}^{\varepsilon}$ induce $\varepsilon$-derivations of degree 1 and -1 respectively.

When the $A$-module $\Omega_{A / k}^{1}$ of first order Kähler differentials has a basis consisting of differentials $d x_{1}, \ldots, d x_{m}$, for some elements $x_{1}, \ldots, x_{m}$ of $A$, we show that the algebra of symmetric differentials is the polynomial ring over $A$ in the infinitely many variables $d^{n} x_{i}$, for $i=1, \ldots, m$ and $n=1,2, \ldots$, and the algebra of alternating differentials is the alternating algebra freely generated by the same differentials.

7.1. Setup. Assume in this section that $A$ is a commutative $k$-algebra and that $\varepsilon^{2}=1$. When $\varepsilon=-1$, an $\varepsilon$-derivation is called a skew derivation and an $\varepsilon$ commutator is called a skew commutator. 
7.2. Assume for a moment that $\varepsilon$ is arbitrary. By (1.7), the endomorphism $\partial_{1}^{\varepsilon}$ is a right $\varepsilon$-derivation of $A\langle t\rangle$, that is, $\partial_{1}^{\varepsilon}(P Q)=P \partial_{1}^{\varepsilon} Q+\left(\partial_{1}^{\varepsilon} P\right) \varepsilon_{\mathrm{gr}} Q$. It is determined in degree $n$ by the formula

$$
\partial_{1}^{\varepsilon}\left(a_{n} t \cdots t a_{0}\right)=\sum_{i=0}^{n-1} \varepsilon^{i} a_{n} t \cdots t a_{i+1} a_{i} t \cdots t a_{0} .
$$

It follows from (2.12) that $\partial_{1}^{\varepsilon}$ vanishes on the subalgebra $\Lambda_{A / k}^{\varepsilon}$ of $A\langle t\rangle$. Denote, for a fixed but arbitrary $\varepsilon$, by $P \mapsto P^{\prime}$ the $k$-linear endomorphism of $A\langle t\rangle$ determined in degree $n$ by the formula

$$
\left(a_{n} t \cdots t a_{0}\right)^{\prime}=\sum_{i=1}^{n-1} i \varepsilon^{i-1} a_{n} t \cdots t a_{i+1} a_{i} t \cdots t a_{0} .
$$

Clearly, the endomorphism $P \mapsto P^{\prime}$ is homogeneous of degree -1 , and left and right $A$-linear. More generally, we have, for $P \in A\langle t\rangle$ and $Q \in A\langle t\rangle^{q}$, the equation

$$
(P Q)^{\prime}=P Q^{\prime}+\varepsilon^{q} P^{\prime} Q+q \varepsilon^{q-1}\left(\partial_{1}^{\varepsilon} P\right) Q .
$$

Indeed, it is easy to deduce (7.2.3) from (7.2.1) and (7.2.2). Alternatively, the polynomial $P^{\prime}$ is obtained by applying formally the operator $(d / d \varepsilon)$ to $\partial_{1}^{\varepsilon} P$, and so (7.2.3) follows from the equation $\partial_{1}^{\varepsilon}(P Q)=P \partial_{1}^{\varepsilon} Q+\varepsilon^{q}\left(\partial_{1}^{\varepsilon} P\right) Q$.

Clearly, $\partial_{1}^{\varepsilon} t=1$ and $t^{\prime}=0$. Hence from (7.2.3) we obtain the equations

$$
(t Q)^{\prime}=t Q^{\prime}+q \varepsilon^{q-1} Q, \quad(Q t)^{\prime}=\varepsilon Q^{\prime} t+\left(\partial_{1}^{\varepsilon} Q\right) t .
$$

If $Q \in \Lambda_{A / k}^{\varepsilon, q}$, then $\partial_{1}^{\varepsilon} Q=0$, and so (7.2.4) implies that

$$
\left(\Delta_{t}^{\varepsilon} Q\right)^{\prime}=\varepsilon^{q-1} q Q+t Q^{\prime}-\varepsilon^{q} \varepsilon Q^{\prime} t .
$$

7.3. Definition. Assume again, as in (7.1), that $\varepsilon^{2}=1$. Let $\delta_{t}^{\varepsilon}: A\langle t\rangle \rightarrow A\langle t\rangle$ be the $k$-linear endomorphism of degree -1 determined in degree $n$ by the formula

$$
\delta_{t}^{\varepsilon}\left(a_{n} t \cdots t a_{0}\right)=\sum_{i=1}^{n-1} i \varepsilon^{n-i-1} a_{n} t \cdots t a_{i+1} a_{i} t \cdots t a_{0} .
$$

Clearly, the endomorphism $\delta_{t}^{\varepsilon}$ is $A$-A-linear. In the notation of (7.2), we have that $\delta_{t}^{\varepsilon}(P)=\left(\varepsilon_{\mathrm{gr}} P\right)^{\prime}$. Hence, for $Q \in \Lambda_{A / k}^{\varepsilon, q}$, it follows from (7.2.5) that

$$
\delta_{t}^{\varepsilon}\left(\Delta_{t}^{\varepsilon} Q\right)=q Q+\varepsilon \Delta_{t}^{\varepsilon}\left(\delta_{t}^{\varepsilon} Q\right)
$$

The subalgebra $\Lambda_{A / k}^{\varepsilon}$ of $A\langle t\rangle$ is the smallest $\Delta_{t}^{\varepsilon}$-invariant $A$-submodule containing 1. Therefore, by induction on the degree $q$, it follows from (7.3.2) that $\Lambda_{A / k}^{\varepsilon}$ is stable under the map $\delta_{t}^{\varepsilon}$. Hence $\delta_{t}^{\varepsilon}$ may be viewed as endomorphism of $\Lambda_{A / k}^{\varepsilon}$, and, for $P \in \Lambda_{A / k}^{\varepsilon}$ and $Q \in \Lambda_{A / k}^{\varepsilon, q}$, we have the equation

$$
\delta_{t}^{\varepsilon} d_{t}^{\varepsilon} Q=q Q+\varepsilon d_{t}^{\varepsilon} \delta_{t}^{\varepsilon} Q
$$

Moreover, from (7.2.3) we obtain, for $P, Q \in \Lambda_{A / k}^{\varepsilon}$, the equation

$$
\delta_{t}^{\varepsilon}(P Q)=\left(\delta_{t}^{\varepsilon} P\right) Q+\left(\varepsilon_{\mathrm{gr}} P\right) \delta_{t}^{\varepsilon} Q .
$$

Thus $\delta_{t}^{\varepsilon}$ is an $\varepsilon$-derivation of $\Lambda_{A / k}^{\varepsilon}$. Since $\delta_{t}^{\varepsilon}$ is $A$-linear, it vanishes on $A=\Lambda_{A / k}^{0}$. In addition, from (7.3.3) it follows that $\delta_{t}^{\varepsilon}$ vanishes on $\Lambda_{A / k}^{1}$. Finally, for $f \in A$, we 
have the equation

$$
\delta_{t}^{\varepsilon}\left(d_{t}^{\varepsilon}\right)^{n} f=\left(\begin{array}{l}
n \\
2
\end{array}\right)_{\varepsilon}\left(d_{t}^{\varepsilon}\right)^{n-1} f .
$$

Indeed, from (1.11.6) it follows easily that $\left(\begin{array}{c}n+1 \\ 2\end{array}\right)_{\varepsilon}-\varepsilon\left(\begin{array}{c}n \\ 2\end{array}\right)_{\varepsilon}=\left(\varepsilon^{2 n}-1\right) /\left(\varepsilon^{2}-1\right)$. In particular, since $\varepsilon^{2}=1$, we have the equation $\left(\begin{array}{c}n+1 \\ 2\end{array}\right)_{\varepsilon}=\varepsilon\left(\begin{array}{c}n \\ 2\end{array}\right)_{\varepsilon}+n$. By using the latter equation and (7.3.3), the equation (7.3.5) follows by induction on $n$.

7.4. Lemma. The $\varepsilon$-derivation $\delta_{t}^{\varepsilon}$ of $\Lambda_{A / k}^{\varepsilon}$ induces an $\varepsilon$-derivation $\delta^{\varepsilon}$ of the algebra of Kähler differentials $\Omega_{A / k}^{\varepsilon}$.

Proof. Recall from (3.2) that the algebra of Kähler differentials is the quotient of $\Lambda_{A / k}^{\varepsilon}$ modulo the ideal $\mathfrak{A}^{\varepsilon}$ consisting of sums of polynomials of the form

$$
W=P\left(d_{t}^{\varepsilon}\right)(\rho Q)
$$

where $P \in A\langle t\rangle, Q \in \Lambda_{A / k}^{\varepsilon}$, and $\rho=\left(\Delta_{t} b\right) a-a\left(\Delta_{t} b\right)$ for $a, b \in A$. It suffices to prove that the ideal $\mathfrak{A}^{\varepsilon}$ is invariant under $\delta_{t}^{\varepsilon}$. So consider the value of $\delta_{t}^{\varepsilon}$ on a polynomial (7.4.1). Clearly, we may assume that $P$ is homogeneous, and by $A$-linearity we may assume either that $P=1$ or that $P=t R$ for some polynomial $R \in A\langle t\rangle$. In the first case we have, since $\delta_{t}^{\varepsilon}$ is an $\varepsilon$-derivation and vanishes on $\Lambda_{A / k}^{1}$, that

$$
\delta_{t}^{\varepsilon} W=\delta_{t}^{\varepsilon}(\rho Q)=\varepsilon \rho \delta_{t}^{\varepsilon} Q,
$$

which clearly is of the form (7.4.1). In the second case we have that

$$
\delta_{t}^{\varepsilon} W=\delta_{t}^{\varepsilon} d_{t}^{\varepsilon} R\left(d_{t}^{\varepsilon}\right)(\rho Q) .
$$

So, using (7.3.3) and induction on the degree of $P$, it follows that $\delta_{t}^{\varepsilon} W$ belongs to $\mathfrak{A}^{\varepsilon}$.

7.5. Proposition. Let $(C, d)$ be a Kähler system with a Kähler basis. Then the endomorphism $\delta^{\varepsilon}$ of $\Omega_{A / k}$ induces an endomorphism $\delta^{\varepsilon}$ of $\Omega(C)$, that is, there is a canonical A-linear map $\delta^{\varepsilon}: \Omega(C) \rightarrow \Omega(C)$, homogeneous of degree -1 , such that the following diagrams are commutative:

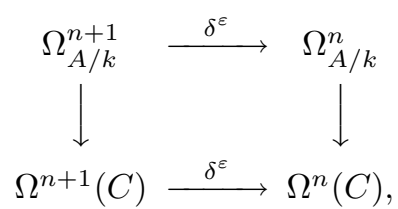

where the vertical maps are the surjections of (5.1.7). Moreover, the induced endomorphism $\delta^{\varepsilon}$ is an $\varepsilon$-derivation of $\Omega(C)$.

Proof. The vertical maps are surjective. Hence the endomorphism $\delta^{\varepsilon}$ of $\Omega(C)$ is uniquely determined by the commutativity of the diagram.

To define $\delta^{\varepsilon}$, let $\mathcal{X}=\left\{x_{1}, \ldots, x_{m}\right\}$ be a Kähler basis for $(C, d)$. The maps $\delta^{\varepsilon}: \Omega^{n+1}(C) \rightarrow \Omega^{n}(C)$ are defined inductively. For $n=0$, let $\delta^{\varepsilon}:=0$. For $n \geq 1$, let $\delta^{\varepsilon}$ be the $A$-linear map given, inductively, on the basis $\mathcal{X}_{n+1}$ of $\Omega^{n+1}(C)$, by the equations

$$
\delta^{\varepsilon}(d \xi)=n \xi+\varepsilon d \delta^{\varepsilon} \xi, \quad \delta^{\varepsilon}\left(d x_{j} . \xi\right)=\varepsilon d x_{j} . \delta^{\varepsilon} \xi \quad \text { for } \xi \in \mathcal{X}_{n} .
$$

First, for $f \in A$ and $\omega \in \Omega^{n}(C)$, we have the equation

$$
\delta^{\varepsilon}(d f \cdot \omega)=\varepsilon d f . \delta^{\varepsilon} \omega .
$$


Indeed, the equation holds trivially if $n=0$. In general, the map $\delta^{\varepsilon}$ is $A$-linear, and the product is $A$-bilinear and $d f$ commutes with the elements of $A$. Therefore, to prove (7.5.3) for $n \geq 1$, we may assume that $d f=d x_{j}$ and that $\omega=\xi$ belongs to $\mathcal{X}_{n}$. Then (7.5.3) follows from the second equation in (7.5.2).

Next, for $\omega \in \Omega^{n}(C)$ we have the equation

$$
\delta^{\varepsilon} d \omega=n \omega+\varepsilon d \delta^{\varepsilon} \omega .
$$

Indeed, (7.5.4) holds trivially when $n=0$. If $n \geq 1$, we may, by additivity, assume that $\omega=f \xi$ for $f \in A$ and $\xi \in \mathcal{X}_{n}$. Then, by (7.5.3) and first equation of (7.5.2), we have the equations for the left side of (7.5.4),

$$
\delta^{\varepsilon} d \omega=\delta^{\varepsilon}(d f . \xi)+\delta^{\varepsilon}(f d \xi)=\varepsilon d f . \delta^{\varepsilon} \xi+f\left(n \xi+\varepsilon d \delta^{\varepsilon} \xi\right)
$$

and, clearly, we have the equations for the right side,

$$
n \omega+\varepsilon d \delta^{\varepsilon} \omega=n f \xi+\varepsilon d \delta^{\varepsilon}(f \xi)=n f \xi+\varepsilon d\left(f \delta^{\varepsilon} \xi\right)=n f \xi+\varepsilon d f . \delta^{\varepsilon} \xi+\varepsilon f d \delta^{\varepsilon} \xi .
$$

Hence the two sides of Equation (7.5.4) are equal.

Consider the diagram (7.5.1). The commutativity is proved by induction on $n$. It holds trivially for $n=0$. Assume that $n \geq 1$. The two maps $\delta^{\varepsilon}$ are $A$-linear and the two vertical maps, denoted $\pi \mapsto \bar{\pi}$, are $A$-linear. Moreover, $\overline{d_{A / k} \pi}=d \bar{\pi}$. Hence, to prove that the diagram is commutative, it suffices to prove, for $\pi \in \Omega_{A / k}^{n}$, that

$$
\delta^{\varepsilon} d \bar{\pi}=\overline{\delta^{\varepsilon} d_{A / k} \pi} .
$$

Apply Equation (7.5.4) to the left side and the analogous equation, deduced from (7.3.3) for the endomorphism $\delta^{\varepsilon}$ of $\Omega_{A / k}$, to the right side. It follows from the induction hypothesis that the two sides are equal.

Hence the diagram (7.5.1) is commutative. Since the map $\pi \mapsto \bar{\pi}$ is a surjective map $\Omega_{A / k}^{\varepsilon} \rightarrow \Omega(C)$ of $k$-algebras over $A$ and $\delta^{\varepsilon}$ is an $\varepsilon$-derivation of $\Omega_{A / k}^{\varepsilon}$, it follows that the induced map $\delta^{\varepsilon}$ is an $\varepsilon$-derivation of $\Omega(C)$.

7.6. Definition. Let $P, Q$ be elements of a graded $k$-algebra $\Gamma$ over $A$. Assume that $P$ is homogeneous of degree $p$. Recall from (1.5) that the $\varepsilon$-commutator $[P, Q]_{\varepsilon}$ is defined by the equation

$$
[P, Q]_{\varepsilon}=P Q-\left(\varepsilon_{\mathrm{gr}}^{p} Q\right) P
$$

It follows easily that

$$
[P, Q R]_{\varepsilon}=[P, Q]_{\varepsilon} R+\left(\varepsilon_{\mathrm{gr}}^{p} Q\right)[P, R]_{\varepsilon} .
$$

In addition, since $\varepsilon^{2}=1$, it follows for any $\varepsilon$-derivation $D$ of $\Gamma$ of degree \pm 1 that

$$
D[P, Q]_{\varepsilon}=[D P, Q]_{\varepsilon}+\left[\varepsilon_{\mathrm{gr}} P, D Q\right]_{\varepsilon} .
$$

It follows from (7.6.1) that the left ideal of $\Gamma$ generated by the $\varepsilon$-commutators is a two sided ideal of $\Gamma$. It is called the $\varepsilon$-commutator ideal. It is obviously a homogeneous ideal. It follows from (7.6.2) that the $\varepsilon$-commutator ideal is $D$ invariant.

Denote by $\mathfrak{C}_{\varepsilon}$ the $\varepsilon$-commutator ideal of the $k$-algebra $\Omega_{A / k}^{\varepsilon}$ and let $\Omega_{A / k}^{\varepsilon \text {-sym }}:=$ $\Omega_{A / k}^{\varepsilon} / \mathfrak{C}_{\varepsilon}$ be the quotient algebra. Then $\Omega_{A / k}^{\varepsilon \text {-sym }}$ is a graded $\varepsilon$-commutative algebra over $A$. Clearly, the algebra could equally have been obtained by dividing $\Lambda_{A / k}^{\varepsilon}$ by its $\varepsilon$-commutator ideal. The elements of the algebra are called $\varepsilon$-symmetric 
differentials. It follows from the considerations above that the $\varepsilon$-derivations $d=$ $d_{A / k}$ and $\delta^{\varepsilon}$ of $\Omega_{A / k}^{\varepsilon}$ induce $\varepsilon$-derivations of $\Omega_{A / k}^{\varepsilon \text {-sym }}$.

When $\varepsilon=1$, the algebra is denoted $\Omega_{A / k}^{\text {sym }}$, and its elements are called symmetric differentials. Clearly, the algebra is commutative. The induced derivations are denoted $d^{\text {sym }}$ and $\delta^{\text {sym }}$, or simply $d$ and $\delta$.

When $\varepsilon=-1$, the algebra is denoted $\Omega_{A / k}^{\text {skew }}$, and its elements are called skew symmetric differentials. Clearly, the algebra is skew commutative. The induced derivations are denoted $d^{\text {skew }}$ and $\delta^{\text {skew }}$, or simply $d$ and $\delta$. The algebra obtained from $\Omega_{A / k}^{\text {skew }}$ by dividing by the ideal generated by all squares of elements of odd degree is denoted $\Omega_{A / k}^{\text {alt }}$. Clearly, the latter ideal is the left ideal generated by the squares of elements of odd degree. Moreover, if $\pi$ is an element of odd degree in $\Omega_{A / k}^{\text {skew }}$, then

$$
d\left(\pi^{2}\right)=d \pi \cdot \pi-\pi \cdot d \pi=[d \pi, \pi]_{-1}=0,
$$

and, similarly, $\delta\left(\pi^{2}\right)=0$. It follows that the ideal in $\Omega_{A / k}^{\text {skew }}$ generated by the squares of elements of odd degree is invariant under $d$ and $\delta$. Hence $d$ and $\delta$ induce derivations in $\Omega_{A / k}^{\text {alt }}$.

Consider a derivation system $(C, d)$. It follows from Lemma (4.2) that $C$ is a module over $\Omega_{A / k}^{\varepsilon}$. Hence the quotient $C^{\varepsilon \text {-sym }}:=C / \mathfrak{C}_{\varepsilon} C$ is a module over $\Omega_{A / k}^{\varepsilon \text {-sym }}$. By (4.3), the endomorphism $d$ of $C$ is an $\varepsilon$-derivation with respect to the action of $\Omega_{A / k}^{\varepsilon}$ on $C$. As noted above, the commutator ideal $\mathfrak{C}_{\varepsilon}$ is invariant under the endomorphism $d_{A / k}$ of $\Omega_{A / k}$. It follows that the endomorphism $d$ of $C$ induces an endomorphism $d$ of the quotient $C^{\varepsilon \text {-sym }}$.

Similarly, the quotient $\Omega^{\varepsilon-\operatorname{sym}}(C):=\Omega(C) / \mathfrak{C}_{\varepsilon} \Omega(C)$ is a module over $\Omega_{A / k}^{\varepsilon \text {-sym }}$, and there is an induced endomorphism $d$ of $\Omega^{\varepsilon \text {-sym }}(C)$.

Clearly, if $\Omega(C)$ has a structure as a $k$-algebra such that the canonical surjection of (5.1.7) is a surjection of algebras $\Omega_{A / k}^{\varepsilon} \rightarrow \Omega(C)$, then $\Omega^{\varepsilon-\text { sym }}(C)$ is obtained from $\Omega(C)$ by division by the $\varepsilon$-commutator ideal of $\Omega(C)$.

Finally, for $\varepsilon=-1$, the $\Omega_{A / k}^{\text {alt }}$-modules $C^{\text {alt }}$ and $\Omega^{\text {alt }}(C)$ are defined similarly.

7.7. Proposition. Assume that $\left\{x_{1}, \ldots, x_{m}\right\}$ is Kähler basis for a Kähler system $(C, d)$. Then the algebra $\Omega^{\mathrm{sym}}(C)$ is the commutative A-algebra freely generated by the infinitely many differentials $d^{n} x_{j}$ for $n=1,2, \ldots$ and $j=1, \ldots, m$. Similarly, the algebra $\Omega^{\text {alt }}(C)$ is the alternating A-algebra freely generated by the differentials $d^{n} x_{j}$.

Proof. By Theorem (6.7), for $\varepsilon=1$, we have that $\Omega(C)$ is a $k$-algebra over $A$. The algebra $\Omega^{\mathrm{sym}}(C)$ is obtained by dividing the algebra $\Omega(C)$ by its commutator ideal $\mathfrak{C}$. Similarly, the algebra $\Omega^{\text {alt }}$ is obtained, for $\varepsilon=-1$, by dividing the algebra $\Omega(C)$ by the ideal $\mathfrak{C}$ generated by all skew commutators and all squares of elements of odd degree.

As noted in (6.8), the set of all $\varepsilon$-products

$$
\pi=d^{n_{1}} x_{j_{1}} \ldots d^{n_{l}} x_{j_{l}}
$$

where each $n_{i}>0$, is a left $A$-basis of $\Omega_{A / k}^{\varepsilon}$. Therefore, to prove the first assertion of the proposition, it suffices to prove, for $\varepsilon=1$, that the ideal $\mathfrak{C}$ is generated as a left $A$-module by all elements of the form

$$
\pi_{1}\left[d^{n} x_{i}, d^{p} x_{j}\right]_{\varepsilon} \pi_{2}
$$


where $\pi_{1}$ and $\pi_{2}$ are products of the form (7.7.1). Similarly, to prove the second assertion of the proposition, it suffices to prove, for $\varepsilon=-1$, that the ideal $\mathfrak{C}$ is generated as a left $A$-module by all elements of the following forms:

$$
\pi_{1}\left[d^{n} x_{i}, d^{p} x_{j}\right]_{\varepsilon} \pi_{2}, \quad \pi_{1}\left(d^{n} x_{i}\right)^{2} \pi_{2},
$$

where $n$ is odd in the second expression.

Consider first $\Omega^{\operatorname{sym}}(C)$. Fix $\varepsilon=1$, and omit $\varepsilon$ in the notation. Let $\mathfrak{D}$ be the $A$-submodule of $\Omega(C)$ generated by the elements (7.7.2). We have to prove that $\mathfrak{D}=\mathfrak{C}$. Obviously, $\mathfrak{D} \subseteq \mathfrak{C}$.

To prove the opposite inclusion, we have to prove that any commutator belongs to $\mathfrak{D}$. First, since the products (7.7.1) generate $\Omega(C)$ as a left $A$-module, it follows that $\mathfrak{D}$ is the left ideal of $\Omega(C)$ generated by all elements

$$
\left[d^{n} x_{i}, d^{p} x_{j}\right] \pi
$$

where $\pi$ is a product of the form (7.7.1). Clearly, $\mathfrak{D}$ is stable under multiplication from the right by a product $\pi$ of the form (7.7.1). Moreover, $\mathfrak{D}$ is $d$-invariant. Indeed, for any product (7.7.4) we have, by (7.6.2), that

$$
d\left(\left[d^{n} x_{i}, d^{p} x_{j}\right] \pi\right)=\left[d^{n+1} x_{i}, d^{p} x_{j}\right] \pi+\left[d^{n} x_{i}, d^{p+1} x_{j}\right] \pi+\left[d^{n} x_{i}, d^{p} x_{j}\right] d \pi,
$$

and clearly, $d \pi$ is a sum of products of the form (7.7.1).

Any commutator is a sum of commutators of the form $\left[f_{1} \pi_{1},, f_{2} \pi_{2}\right]$ for $f_{1}, f_{2} \in A$ and products $\pi_{1}, \pi_{2}$ of the form (7.7.1). Thus it suffices to prove that $\left[f_{1} \pi_{1}, f_{2} \pi_{2}\right]$ belongs to $\mathfrak{D}$.

Since $f_{1} f_{2}=f_{2} f_{1}$, it follows that

$$
\left[f_{1} \pi_{1}, f_{2} \pi_{2}\right]=f_{1} \pi_{1} f_{2} \pi_{2}-f_{2} \pi_{2} f_{1} \pi_{1}=f_{1}\left[\pi_{1}, f_{2}\right] \pi_{2}-f_{2}\left[\pi_{2}, f_{1}\right] \pi_{1}+f_{1} f_{2}\left[\pi_{1}, \pi_{2}\right] .
$$

Therefore, it suffices to prove that every commutator of the form $[\pi, f]$ or the form $\left[\pi_{1}, \pi_{2}\right]$ belongs to $\mathfrak{D}$. In the commutator $\left[\pi_{1}, \pi_{2}\right]=\pi_{1} \pi_{2}-\pi_{2} \pi_{1}$, we have that $\pi_{1} \pi_{2}$ is a product of factors $d^{n} x$ and $\pi_{2} \pi_{1}$ is the product of the same factors in a different order. Since any permutation can be obtained by successive interchange of neighbors, it follows that $\left[\pi_{1}, \pi_{2}\right]$ is a sum of commutators of the form (7.7.2). Hence $\left[\pi_{1}, \pi_{2}\right]$ belongs to $\mathfrak{D}$.

Hence is suffices to prove that every commutator $[\pi, f]$ belongs to $\mathfrak{D}$. The proof is by induction on the degree $p$ of $\pi$. For $p=0$ or $p=1$, the commutator is equal to 0 , and so the assertion holds. Assume that $p \geq 2$.

If $\pi$ is decomposable as a product $\pi_{1} \pi_{2}$, that is, when $l$ in (7.7.1) is at least 2 , then, by (7.6.1),

$$
[\pi, f]=\pi_{1}\left[\pi_{2}, f\right]+\left[\pi_{1}, f\right] \pi_{2} .
$$

By induction, the two commutators on the right belong to $\mathfrak{D}$. Since $\mathfrak{D}$ is a left ideal and stable under multiplication from the right by products $\pi$, it follows that the right side belongs to $\mathfrak{D}$. Hence $[\pi, f]$ belongs to $\mathfrak{D}$.

Finally, assume that $\pi=d^{p} x_{k}$ Then, by (7.6.2),

$$
[\pi, f]=\left[d^{p} x_{k}, f\right]=d\left[d^{p-1} x_{k}, f\right]-\left[d^{p-1} x_{k}, d f\right] .
$$

Since $\mathfrak{D}$ is $d$-invariant, it follows by induction that the first term on the right belongs to $\mathfrak{D}$. For the second term use an expansion $d f=\sum_{j=1}^{m} g_{j} d x_{j}$. Then the second 
term is a sum of commutators of the form $\left[d^{p-1} x_{k}, g d x_{j}\right]$ and it suffices to prove that $\left[d^{p-1} x_{k}, g d x_{j}\right]$ belongs to $\mathfrak{D}$. However, by (7.6.1),

$$
\left[d^{p-1} x_{k}, g d x_{j}\right]=\left[d^{p-1} x_{k}, g\right] d x_{j}+g\left[d^{p-1} x_{k}, d x_{j}\right] .
$$

On the right, the first commutator belongs to $\mathfrak{D}$ by induction and, clearly, the second commutator is of the form (7.7.2). So, since $\mathfrak{D}$ is a left $A$-module and stable under multiplication from the right by $d x_{j}$, it follows that $\left[d^{p-1} x_{k}, g d x_{j}\right]$ belongs to $\mathfrak{D}$.

Hence we have proved that any commutator belongs to $\mathfrak{D}$, and the proof of the first assertion of the proposition is complete.

The proof of the second assertion is analogous.

7.8. Note. Assume the conditions of (7.7). By Proposition (7.5), there is an induced $\varepsilon$-derivation $\delta^{\varepsilon}$ of $\Omega(C)$. For $\varepsilon=1$, the algebra $\Omega^{\mathrm{sym}}(C)$ is obtained by division of $\Omega(C)$ with the commutator. Since the commutator ideal is invariant under any derivation, it follows that $\delta^{\varepsilon}$ induces a derivation of degree -1 in $\Omega^{\mathrm{sym}}(C)$.

Similarly, for $\varepsilon=-1$, there are induced skew derivations $\delta^{\varepsilon}$ of degree -1 in $\Omega^{\text {skew }}(C)$ and $\Omega^{\text {alt }}(C)$.

The endomorphisms $d$ and $\delta^{\varepsilon}$ are derivations of the algebra $\Omega_{A / k}^{\mathrm{sym}}$ and skew derivations of the algebra $\Omega_{A / k}^{\text {alt }}$. Note that the endomorphisms are determined on the basis given in (7.6) as follows: For the endomorphism $d$, we have that $d f$ is an $A$-linear combination of the $d x_{j}$, and $d\left(d^{n} x_{j}\right)=d^{n+1} x_{j}$. For the endomorphism $\delta^{\varepsilon}$, we have that $\delta f=0$ and the value $\delta\left(d^{n} x_{j}\right)$ is determined by (7.3.5).

7.9. Proposition. The (left) ideal $\mathfrak{N}$ of $\Omega_{A / k}^{\text {alt }}$ generated by elements $d^{2}(\omega)$ for all $\omega$ in $\Omega_{A / k}^{\mathrm{alt}}$ is homogeneous and d-invariant. Moreover, if $\bar{d}$ denotes the skew derivation induced by $d$ in the quotient $\Omega_{A / k}^{\mathrm{alt}} / \mathfrak{N}$, then the quotient is generated as an $A$-module by all products $\bar{d} f_{1} \cdots \bar{d} f_{n}$ for $f_{1}, \ldots, f_{n} \in A$ and $n=0,1, \ldots$ In fact, the quotient $\Omega_{A / k}^{\text {alt }} / \mathfrak{N}$ is canonically isomorphic to the exterior algebra $\wedge \Omega_{A / k}^{1}$.

Proof. The algebra $\Omega_{A / k}^{\text {alt }}$ is alternating, so the left ideal $\mathfrak{N}$ is an ideal. Moreover, the ideal is $d$-invariant, since

$$
d\left(\pi d^{2} \omega\right)=d \pi d^{2} \omega+\left(\varepsilon_{\mathrm{gr}} \pi\right) d^{2}(d \omega) .
$$

It follows that the quotient $\Omega_{A / k}^{\text {alt }} / \mathfrak{N}$ is a graded alternating algebra and that there is an induced skew derivation $\bar{d}$ of the quotient. The elements of degree $n+1$ of the quotient are $A$-linear combinations of elements of the form $\bar{d} \pi$, where $\pi$ is of degree $n$. Since $\bar{d}^{2}=0$, it follows that

$$
\bar{d}\left(f \bar{d} f_{1} \cdots \bar{d} f_{n}\right)=\bar{d} f \bar{d} f_{1} \cdots \bar{d} f_{n} .
$$

So, by induction on $n$, the products $\bar{d} f_{1} \cdots \bar{d} f_{n}$ generate the degree- $n$ part of $\Omega_{A / k}^{\text {alt }} / \mathfrak{N}$.

The exterior algebra $\bigwedge \Omega_{A / k}^{1}$ is alternating. It is well known that the module derivation $d: A \rightarrow \Omega_{A / k}^{1}$ extends to a skew derivation $\tilde{d}$ of the exterior algebra. Moreover, $\tilde{d}^{2}=0$. It follows that there is a canonical homomorphism of algebras over $A$ with $k$-linear endomorphisms,

$$
\left(\Omega_{A / k}^{\text {alt }} / \mathfrak{N}, \bar{d}\right) \rightarrow\left(\bigwedge \Omega_{A / k}^{1}, \tilde{d}\right) .
$$


On the other side, since $\Omega_{A / k}^{\text {alt }} / \mathfrak{N}$ is alternating and equal to $\Omega_{A / k}^{1}$ in degree 1 , there is a canonical homomorphism of algebras

$$
\bigwedge \Omega_{A / k}^{1} \rightarrow \Omega_{A / k}^{\text {alt }} / \mathfrak{N} \text {. }
$$

Clearly, it follows from the analysis in the first part of the proof that (7.9.1) is an isomorphism with (7.9.2) as inverse.

7.10. Example. The constructions given in this section apply to the system of Meyer considered in the examples (5.10) and (6.10). Moreover, the constructions apply to the Semple systems considered in examples (5.9) and (6.9) when the quotient $Q$ has an $A$-basis of the form $d x_{j}$. In particular, when $\Omega_{A / k}^{1}$ has a basis of the form $d x_{j}$, the constructions apply to the non-commutative version of Iitaka's differentials. Moreover, as noted in (5.7), for the system considered by Iitaka, we have that $\Omega_{A / k}^{\mathrm{Iit}}=\Omega_{A / k}^{\mathrm{sym}}$.

\section{REFERENCES}

1. A. Buium, Intersections in jet spaces and a conjecture of S. Lang, Ann. of Math. 136 (1992), 557-567. MR 93j:14055

2. A. Buium, Geometry of differential polynomial functions, I: algebraic groups, Amer. J. Math. 115 (1993), 1385-1444. MR 95c:12011

3. A. Buium, Geometry of differential polynomial functions, II: algebraic curves, Amer. J. Math. 116 (1994), 785-818. MR 96a:14039

4. A. Buium, Geometry of differential polynomial functions, III: moduli spaces, Amer. J. Math. 117 (1995), 1-73. MR 96b:14059

5. S. J. Colley and G. Kennedy, A higher-order contact formula for plane curves, Comm. Algebra 19(2) (1991), 479-508. MR 92f:14056

6. S. J. Colley and G. Kennedy, The enumeration of simultaneous higher-order contacts between plane curves, Compositio Math. 93(2) (1994), 171-206. MR 95f:14097

7. A. Collino, Evidence for a conjecture of Ellingsrud and Strömme on the Chow ring of $\mathrm{Hilb}_{d}\left(\mathbf{P}^{2}\right)$, Illinois J. Math. 32 (1988), 171-210. MR 89d:14004

8. J.-P. Demailly, Algebraic criteria for Kobayashi hyperbolic projective varieties and jet differentials, Lecture Notes, AMS Summer Research Institute Santa Cruz 1995, PSPM, vol. 62, part 2, 1997. CMP 98:07

9. A. Grothendieck, with J. Dieudonné, Eléments de Géométrie Algébrique $I V_{4}$, Inst. Hautes Études Sci. Publ. Math. 32 (1967), 1-361. MR 39:220

10. S. Iitaka, Symmetric forms and Weierstrass cycles, Proc. Japan Acad., Ser. A 54 (1978), 101-103. MR 58:10893

11. S. Iitaka, Duality theorems for symmetric differential forms, Proc. Jap. Acad., Ser. A 55 (1979), 53-58. MR 80j:14018

12. S. Iitaka, Symmetric forms and Weierstrass semigroups, Algebraic geometry, Proc. Summer Meet., Copenh. 1978, Lect. Notes Math. 732, 1979, pp. 157-170. MR 81e:14012

13. S. Iitaka, Weierstrass forms associated with linear systems, Adv. Math. 33 (1979), 14-30. MR 81c: 14003

14. J. Johnson, Order for systems of differential equations and a generalization of the notion of differential ring, J. Algebra 78 (1982), 91-119. MR 85i:12010

15. J. Johnson, Prolongations of integral domains, J. Algebra 94 (1985), 173-210. MR 86i:12008

16. E. R. Kolchin, Differential algebra and algebraic groups, Academic Press, 1973. MR 58:27929

17. E. R. Kolchin, Differential algebraic groups, Academic Press, New York, 1985. MR 87i:12016

18. D. Laksov and A. Thorup, The Brill-Segre formula for families of curves, Enumerative Algebraic Geometry: Proceedings of the 1989 Zeuthen Symposium in Copenhagen, S. L. Kleiman and A. Thorup (Eds.), Contemporary Mathematics 123, AMS, Providence, 1991, pp. 131-148. MR 92k: 14029

19. D. Laksov and A. Thorup, Weierstrass points and gap sequences for families of curves, Ark. Mat. 32 (1994), 393-422. MR 96b:14041 
20. D. Laksov and A. Thorup, Weierstrass points on schemes, J. Reine Angew. Math. 460 (1995), 127-164. MR 96b:14016

21. P.-A. Meyer, Formes differentielles d'ordre $n>1$, Publication IRMA, Université Louis Pasteur, Strasbourg, 1979/80.

22. P.-A. Meyer, Qu'est ce qu'une différentielle d'ordre n?, Exposition. Math. 7 (1989), 249-264. MR 91d:60125

23. J. F. Ritt, Differential Algebra, Amer. Math. Soc. Coll. Publ. 33, Amer. Math. Soc., New York, 1950. MR 12:7c

24. R. Speiser, Derived triangles and differential systems, Projective geometry with applications, Lecture Notes in Pure and Applied Mathematics, 166, Dekker, New York, 1994, pp. 97-109. MR 95k:14073

KTH, S-100 44 Stockholm, Sweden

E-mail address: laksov@math.kth.se

Matematisk Afdeling, Københavns Universitet, Universitetsparken 5, DK-2100 København $\varnothing$, Denmark

E-mail address: thorup@math.ku.dk 